\title{
Risk management from a microeconomic perspective
}

Citation for published version (APA):

Csóka, P. (2008). Risk management from a microeconomic perspective. [Doctoral Thesis, Maastricht University]. Universitaire Pers Maastricht. https://doi.org/10.26481/dis.20081030pc

Document status and date:

Published: 01/01/2008

DOI:

$10.26481 /$ dis.20081030pc

Document Version:

Publisher's PDF, also known as Version of record

\section{Please check the document version of this publication:}

- A submitted manuscript is the version of the article upon submission and before peer-review. There can be important differences between the submitted version and the official published version of record.

People interested in the research are advised to contact the author for the final version of the publication, or visit the DOI to the publisher's website.

- The final author version and the galley proof are versions of the publication after peer review.

- The final published version features the final layout of the paper including the volume, issue and page numbers.

Link to publication

\footnotetext{
General rights rights.

- You may freely distribute the URL identifying the publication in the public portal. please follow below link for the End User Agreement:

www.umlib.nl/taverne-license

Take down policy

If you believe that this document breaches copyright please contact us at:

repository@maastrichtuniversity.nl

providing details and we will investigate your claim.
}

Copyright and moral rights for the publications made accessible in the public portal are retained by the authors and/or other copyright owners and it is a condition of accessing publications that users recognise and abide by the legal requirements associated with these

- Users may download and print one copy of any publication from the public portal for the purpose of private study or research.

- You may not further distribute the material or use it for any profit-making activity or commercial gain

If the publication is distributed under the terms of Article $25 \mathrm{fa}$ of the Dutch Copyright Act, indicated by the "Taverne" license above, 
Risk Management from a Microeconomic Perspective 
(C) Péter Csóka, Maastricht 2008

All rights reserved. No part of this publication may be reproduced, stored in a retrieval system, or transmitted in any form, or by any means, electronic, mechanical, photocopying, recording or otherwise, without the prior permission in writing from the author.

The chart in the cover displays the HUF/EUR time series between September 2004 and August 2008. The vertical axis is from 220 to 290 HUF/EUR. Source: www.mnb.hu.

ISBN 978-90-5278-769-5

Published by Universitaire Pers Maastricht

Printed in the Netherlands by Datawyse Maastricht

This book was typeset by the author using $\mathrm{ET}_{\mathrm{E}} \mathrm{X}$. 


\section{Risk Management from a Microeconomic Perspective}

\section{PROEFSCHRIFT}

ter verkrijging van de

graad van doctor aan de Universiteit Maastricht, op gezag van de

Rector Magnificus, Prof. mr. G.P.M.F. Mols, volgens het besluit van

het College van Decanen, in het openbaar te verdedigen op

donderdag 30 oktober 2008 om 14.00 uur

door

Péter Csóka

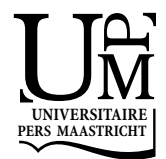




\section{Promotor}

Prof. Dr. P.J.J. Herings

\section{Copromotor}

Dr. L. Á. Kóczy (Maastricht University and Budapest Tech)

\section{Assessment Committee}

Prof. Dr. H.J.M. Peters (chairman)

Prof. Dr. P.E.M. Borm (Tilburg University)

Dr. A. Predtetchinski 


\section{Acknowledgements}

Microeconomics was my favorite subject at Sándor Petőfi Secondary School of Economics. The teaching method of Erzsébet Diószegi made sure that I have learned the basic intuitions.

There was no doubt that I wanted to study economics in a university. I could learn the advanced concepts at Budapest University of Economic Sciences and Public Administration (BUESPA, now Corvinus University). Special thanks to János Száz for raising my interest in finance; to Ernő Zalai and Imre Csekő for showing me the beauty in general equilibrium theory; to Ferenc Forgó and Tamás Solymosi for teaching me game theory; to my master's thesis supervisor Júlia Király for encouraging me to do a Ph. D.; and to József Móczár for leading me in my first year of doctoral studies.

How to do state of the art research was shown to me by my supervisors at Maastrict University:

P. Jean-Jacques Herings and László Á. Kóczy. After our long brainstorming meetings I always had enough munition to write, they always checked the papers carefully and I was given detailed comments. I would also like to thank László for his support to get started and to feel at home throughout the years in Maastricht.

I would like to thank Hans Peters, Peter Borm and Arkadi Predtetchinski for being part of my assessment committee and giving helpful comments; Helga Habis and Michael Yang for being my paranymphen and helping in organizing my defense.

All financial support from the Faculty of Economics and Business adminstration is gratefully acknowledged. I could visit various conferences and I could deepen my understanding of Economic Theory and Industrial Organizations by doing two courses offered by the Netherlands Network of Economics.

I really enjoyed teaching in the problem based learning system in Maastricht. I would like to thank the cooperation with the course coordinators: Christian Kerckhoffs (Microeconomics); Bettina Klaus and Kristof Bosmans (Design of tax systems). 
My colleagues provided a very nice working atmosphere in Maastricht. During the day, in lunch brakes, in our spare time I could always rely on one or more of them. I just want to name my office mates: I enjoyed sharing my office with Kasper Leufkens, who helped me a lot with his sarcastic and funny personality. It was a pity that Burak Can and Elena Cettolin joined us only temporarily.

My life in Maastricht was enriched by all kinds of meetings and excursions with members of the Hungarian community around it. It was also a pleasure to share my kitchen and ideas with Péter Lukács and Jasper de Boer.

Finally I would like to thank my girlfriend Renáta and my family for their support from home.

Péter Csóka

Maastricht August 29, 2008 


\section{Table of Contents}

$\begin{array}{ll}\text { Acknowledgements } & \text { i }\end{array}$

Table of Contents iii

List of Tables $\quad$ v

List of Figures $\quad$ vii

1 Introduction 1

1.1 General Equilibrium Theory $\ldots \ldots \ldots \ldots 2$

1.2 Cooperative Game Theory . . . . . . . . . . . . . . . . . 3

2 Coherent Measures of Risk from a General Equilibrium Perspective 7

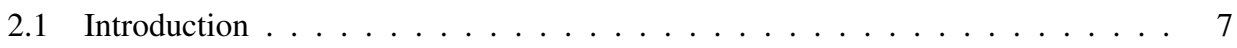

2.2 Coherent Measures of Risk . . . . . . . . . . . . . . . . . . . . . . 9

2.3 Spectral Measures of Risk f . . . . . . . . . . . . . . . . . . . 11

2.4 An Exchange Economy Model . . . . . . . . . . . . . . . . . . . . . 17

2.5 General Equilibrium Measures of Risk . . . . . . . . . . . . . . . 20

2.6 Connections: Spectral and GE Measures of Risk . . . . . . . . . . . . . . . 23

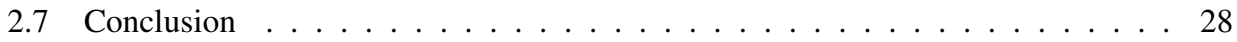

3 Balancedness Conditions for Exact Games $\quad 31$

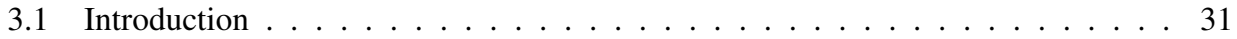

3.2 Notation and Definitions $\ldots \ldots \ldots \ldots \ldots$

3.3 Exact Games and Balancedness . . . . . . . . . . . . . . . . . . . . 34

3.4 Applications of Exact Balancedness . . . . . . . . . . . . . . 40 


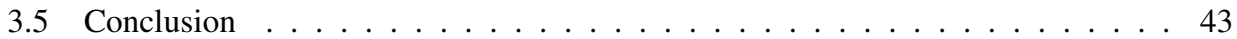

4 Stable Allocations of Risk 45

4.1 Introduction . . . . . . . . . . . . . . . . . . 45

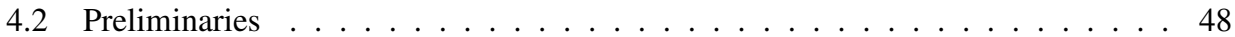

4.2.1 Coherent Measures of Risk . . . . . . . . . . . . . . . . . . . . 48

4.2 .2 Transferable Utility Games . . . . . . . . . . . . . . . . . . . . . . . 49

4.2 .3 Risk Allocation Games . . . . . . . . . . . . . . . . . . . . . . 51

4.3 Total Balancedness . . . . . . . . . . . . . . . . . . . . . 52

4.3.1 Risk Allocation Games and Totally Balanced Games . . . . . . . . . . . 52

4.3.2 Linear Programming Results . . . . . . . . . . . . . . . . . . . 56

4.4 Exactness ........................... 60

4.5 Conclusion ........................ 63

5 Convex and Exact Games with Non-transferable Utility 65

5.1 Introduction . . . . . . . . . . . . . . . . . . . 65

5.2 Notation, Definitions, Existing Results . . . . . . . . . . . . . . . 66

5.2.1 Transferable Utility Games . . . . . . . . . . . . . . . . . . . 67

5.2.2 Non-transferable Utility Games . . . . . . . . . . . . . . . . . . . 69

5.3 Exact NTU Games . . . . . . . . . . . . . . . . . . . . 73

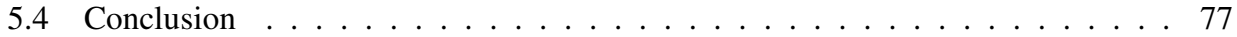

$\begin{array}{ll}\text { Summary } & 81\end{array}$

$\begin{array}{ll}\text { Bibliography } & 85\end{array}$

$\begin{array}{ll}\text { Index } & 91\end{array}$

$\begin{array}{lr}\text { Short Curriculum Vitae } & 95\end{array}$ 


\section{List of Tables}

2.1 Spectral measures of risk with equiprobable outcomes. . . . . . . . . . . . 17

2.2 Spectral measures of risk with not equiprobable outcomes. . . . . . . . . . . . 17

2.3 A GE measure of risk which is not law invariant. . . . . . . . . . . . . 22

2.4 A GE measure of risk and the CAPM formula. . . . . . . . . . . . . . . . 22

$2.5 Z$ is not comonotonic with $\widetilde{\omega} \ldots \ldots \ldots \ldots \ldots \ldots$

4.1 A totally balanced game and its zero-normalized game. . . . . . . . . . . . . 55

4.2 Payoff matrices for the zero normalized and the original games. . . . . . . . . 55

4.3 The value function of a convex game $v \ldots \ldots \ldots \ldots$. . . . . . . . 62

4.4 A matrix of realization vectors generating $v \ldots \ldots \ldots$. . . . . . . . 62 


\section{List of Figures}

3.1 Subsets of balanced games. . . . . . . . . . . . . . . . . 44

5.1 Subsets of $\Pi$-balanced games. . . . . . . . . . . . . . . . . 79 


\section{Chapter 1}

\section{Introduction}

Risk management is of crucial importance when we consider the enormous financial risk our economy is exposed to. The risks of many economic agents are regulated by various institutions. External regulators at an international level are the International Actuarial Association (IAA) and the International Accounting Standards Board (IASB), who determine the capital requirements for insurance companies. Similarly, the Basel Committee gives guidelines for the acceptable level of capital on banking supervision. Since a government or central bank could be a lender of last resort for these institutions, and their default could cause serious problems, they are regulated as well. To give a recent example, I think inadequate assessment of risk played a crucial role in the current subprime mortgage crisis as well.

As an internal regulator, a portfolio manager has to regulate the risk of its traders. In the context of a multi-division firm setting, the head-office may also set risk-limits for the divisions. Internally the risk values can also be used for planning and performance evaluation. It is therefore crucial to measure risk in an appropriate way.

In the sequel we will use the term portfolio when referring to any risky entity (firm, bank, insurance company, etc.). A measure of risk assigns a real number to the probability distribution of the future value of a portfolio. It can be interpreted as the minimal amount of cash the regulated agent has to add to his portfolio, and to invest in a zero coupon bond for its risk to be acceptable to the regulator. The literature knows of numerous possible ways to measure risk; lately interest shifted to the axiomatic approach of coherent measures of risk (Artzner, Delbaen, Eber, and Heath, 1999). A coherent measure of risk (Definitions 2.1, 4.1) satisfies the following four axioms: monotonicity, subadditivity, positive homogeneity and translation invariance. Adding two more axioms, comonotonic additivity and law invariance, one obtains a subclass of coher- 
ent measures of risk, called spectral measures of risk (Acerbi, 2002), (Definitions 2.2, 4.2). For an introduction to risk measures and the aforementioned axioms, see Chapter 4 of Föllmer and Schied (2002). Of the axioms, subadditivity and law invariance are the most important ones to mention here. Subadditivity requires that the risk of an aggregate portfolio should not exceed the total risk of the individual subportfolios: it captures the notion of diversification; law invariance holds if the risk of a portfolio depends only on its probability distribution. Subadditivity implies that the risk of the aggregate portfolio is less than the sum of the risks of the individual portfolios. The diversification benefits should be allocated somehow, preferably in a stable way, when no collection of individual portfolios would be better off if they separate from the others. Two natural questions arise:

1. Are coherent and spectral measures of risk a good way to measure risk?

2. How to allocate risk?

This thesis is devoted to the study of those questions from a microeconomic perspective, using tools from general equilibrium theory and cooperative game theory.

\subsection{General Equilibrium Theory}

General equilibrium (GE) theory captures the notion that markets are interrelated. If we want to take into account those dependencies, then it is not enough to consider a standard supply and demand partial equilibrium model. An exchange economy in a dynamic GE model consists of a number of consumers maximizing their utility over a finite number of time periods. Production decisions are assumed to be exogenously given, thus there are no firms in such models. In each time period there is uncertainty about the state of nature to be realized, which determines the amount of goods and services, called commodities a consumer will be endowed with. As an example, you can think of a farmer and the weather caused uncertainties in the production of crops.

Consumers can exchange commodities in each time period and they can also trade with assets before a particular state of nature is realized. An asset specifies the amount of commodities their owner will get depending on the realized state of nature. A zero coupon bond, a stock of a firm, even options can be modeled as an asset. A zero coupon bond provides the same amount of 
money in all states of nature; a stock as an asset specifies the stock price in each state of nature. A call option gives its owner the right (but not the obligation) of buying a stock at a given price (called strike price) at a certain time. A call option as an asset provides zero payoff if the stock price is below the strike price, and the difference between the stock price and the strike price when the stock price is above the strike price.

When consumers are assumed to be price takers, then a consumption bundle, prices of commodities and assets constitute a competitive equilibrium (Definition 2.14) if there is no excess supply or excess demand in any commodities or assets.

In Chapter 2 we analyze the axioms of coherent measures of risk using an exchange economy. We consider one representative consumer, where the stochastic endowment of the consumer represents the market portfolio. This approach has the advantage that it recognizes the fact that the risk of a portfolio depends on the market portfolio, an insight that is generated immediately by the Capital Asset Pricing Model (CAPM) as developed by Sharpe (1964) and Lintner (1965). By doing so we contribute to the research agenda that connects finance to GE theory, see for instance Geanakoplos and Shubik (1990), Magill and Quinzii (1996), Leroy and Werner (2001), or Jaschke and Küchler (2001). The corresponding measure of risk of a portfolio is the amount of cash needed to sell the risk involved in the portfolio to the market. More precisely, the so-called GE measure of risk (Definition 2.18) of a portfolio is the negative of its equilibrium market price in the exchange economy.

We prove that the GE measure of risk is a coherent measure of risk (Proposition 2.20), thus coherent measures of risk are compatible with a natural general equilibrium approach to measure risk. However, using the insight of the CAPM that the risk of a portfolio does not only depend on the probability distribution of its payoff, but also on how these payoffs are correlated to those of the market portfolio, we show that the GE measure of risk does not satisfy law invariance (Example 2.21), and is therefore not a spectral measure of risk.

\subsection{Cooperative Game Theory}

We model the problem of risk allocation by cooperative games. A noncooperative game consists of the set of players, their strategies and the payoffs depending on the strategies selected by the players. In cooperative games strategies are implicit, they are expressed by considering groups 
of players, called coalitions. Payoffs are typically given to these coalitions rather than to the individual players. The solution of such a game must therefore specify the distribution of these payoffs. Unlike in noncooperative games, players can make binding commitments, or contracts that also determine the payoff allocation.

A transferable utility (TU) game originating from the famous book by von Neumann and Morgenstern (1944) consists of a finite set of players and a value function specifying the maximum attainable utility (money) for all the coalitions of players. The core of a TU game consists of those efficient allocations which are robust against all coalitional deviations. It is well-known that the core in a TU game is non-empty if and only if the game is balanced (Bondareva, 1963; Shapley, 1967). One interpretation of balancedness (Definition 3.1) is that the players can distribute one unit of working time to any coalition and in doing so cannot generate more value than the grand coalition, where everybody cooperates with everybody.

One obtains a subgame by restricting the game to a subset of players. The core of every subgame of a TU game is non-empty if and only if the game is totally balanced (Definition 3.2). Totally balanced games arise from a wide range of applications: they coincide with market games (Shapley and Shubik, 1969), they are are generated by linear production games, they are equivalent to a class of maximum flow problems (Kalai and Zemel, 1982a) and also to permutation games of less than four players (Tijs, Parthasarathy, Potters, and Prassad, 1984).

In Chapter 3 we provide a set of linear programming problems to study a subclass of totally balanced games, exact games (Schmeidler, 1972), (Definition 3.3). A game is exact if for each coalition there is a core allocation such that the coalition only gets its stand-alone value. By the linear programming problems one can easily check whether a game is exact or not. Using the dual of the linear programming problems we develop two new characterizations of exact games, complementing earlier characterizations by Schmeidler (1972) and Azrieli and Lehrer (2005).

First, we show (Theorem 3.11) that a game is exact if an only if it is exactly balanced (Definition 3.9). The condition of exact balancedness is identical to the one of balancedness, except for the following. In exact balancedness it holds that even if players in one coalition are allowed to work overtime in any combination of the coalitions, their best choice is still to stick to their original coalition. We show that exact balancedness implies total balancedness.

The second characterization (Theorem 3.13) spells out what more than total balancedness is needed to obtain exactness. It says that a game is exact if an only if it is totally balanced and 
overbalanced (Definition 3.12). In case of overbalancedness, no weight is put on the grand coalition and one coalition works a non-negative amount of overtime. From this characterization it follows immediately that an exact game is totally balanced. The characterizations can be used to verify exactness of a game, we apply them in Chapter 4.

In Chapter 4 we come back to the question of the distribution of the risk diversification benefits. Risk allocation games (Denault, 2001), (Definition 4.12) are transferable utility cooperative games defined to this purpose. Note that we have found the axioms of coherent measures of risk (Definitions 2.1,4.1) compatible with a natural general equilibrium approach to measure risk in Chapter 2. A risk allocation game arises from a risk environment specifying a number of portfolios and a coherent measure of risk determining the risk of each portfolio. Coalitions of agents can combine their portfolios and thereby create diversification gains.

We prove (Theorem 4.17) that the class of risk allocation games also coincides with the class of totally balanced games. This result ensures that a regulator can always allocate risk in a stable way. No matter how the risk environment changes, there is always an allocation of risk that no coalition of portfolios can object to.

To get exact games, we need an extra condition, since exact games are a subclass of totally balanced games. Usually the risk of the aggregate portfolio is low compared to the risk involved in the individual portfolios. As an extreme case, no aggregate uncertainty refers to the case when the value of the aggregate portfolio is constant over all states of nature. We prove (Theorem 4.23) that the class of risk allocation games with no aggregate uncertainty coincides with the class of exact games. To show that all risk allocation games with no aggregate uncertainty are exact, we use the overbalancedness condition developed in Chapter 3.

Thus, in the case of no aggregate uncertainty, for each coalition of portfolios, this coalition does not necessarily benefit from the diversification opportunities offered by the aggregate portfolio. As a consequence, the regulator has a high level of discretion in allocating the risk to the individual portfolios in this case.

The results that the class of risk allocation games in general coincides with the class of totally balanced games, and with no aggregate uncertainty we get the class of exact games are extremely useful in establishing the general properties of standard solution concepts used in cooperative game theory when applied to risk allocation games.

Convex games with transferable utility (Definition 4.10) introduced by Shapley (1971) provide a 
further refinement of exact games, since convex games are a subset of exact games. They arise from a wide range of applications. Airport games (Littlechild and Owen, 1973), bankruptcy games (Aumann and Maschler, 1985), sequencing games (Curiel, Pederzoli, and Tijs, 1989) and standard tree games (Granot, Maschler, Owen, and Zhu, 1996) are all convex. If the number of players (individual portfolios) below four, we show that one obtains the class of convex games in risk allocation games with no aggregate uncertainty (Theorem 4.24).

The assumption of transferable utility can be relaxed to obtain the class of games with nontransferable utility (NTU games, for short). In a TU game it is assumed that utilities can be transferred. Such an assumption is justified if there is a commodity which has the same marginal utility for everyone and the utility functions are linear and separable in it. In general, this is not the case and one would like to study the more general class of NTU games. An NTU game specifies the set of attainable utility levels for the members of each coalition.

The notion of convexity can be generalized to NTU games in at least five ways. Vilkov (1977) and Sharkey (1981) have extended convexity to NTU games to define ordinal (Definition 5.8) and cardinal convexity (Definition 5.9), respectively. Hendrickx, Borm, and Timmer (2002) analyze coalition merge convexity (Definition 5.11), individual merge convexity (Definition 5.12), and marginal convexity (Definition 5.13) in an NTU setting.

Ordinally convex NTU games have numerous applications. Peleg (1984) transforms a social choice situation with a convex effectivity function into an NTU game which is ordinally convex. Demange (1987) provides two examples: a model of public good, and a production economy with increasing returns to scale; Masuzawa (2003) adds $N$-person prisoners' dilemma games and oligopoly models to this class.

The aforementioned five classes of NTU convex games do not coincide in general. The only general result is that coalition merge convexity implies individual merge convexity, and individual merge convexity implies marginal convexity. It is natural to seek a result that is analogous to convex TU games being exact. In Chapter 5 we generalize exactness to the NTU setting. In an exact NTU game (Definition 5.16) for each coalition there is a core element on the boundary of its payoff set, meaning that this coalition does not necessarily benefit from the gains of forming the grand coalition in an allocation which is robust against all coalitional deviations. We show (Theorem 5.22, Corollary 5.24) that each of ordinal, coalition merge, individual merge and marginal convexity can be unified under NTU exactness. 


\section{Chapter 2}

\section{Coherent Measures of Risk from a General Equilibrium Perspective}

Coherent measures of risk defined by the axioms of monotonicity, subadditivity, positive homogeneity, and translation invariance are recent tools in risk management to assess the amount of risk agents are exposed to. If they also satisfy law invariance and comonotonic additivity, then we get a subclass of them: spectral measures of risk. Expected shortfall is a well-known spectral measure of risk.

In this chapter ${ }^{1}$ we investigate the above mentioned six axioms using tools from general equilibrium (GE) theory. Coherent and spectral measures of risk are compared to the natural measure of risk derived from an exchange economy model, which we call the GE measure of risk. We prove that GE measures of risk are coherent measures of risk. We also show that spectral measures of risk are GE measures of risk only under stringent conditions, since spectral measures of risk do not take the regulated entity's relation to the market portfolio into account. To give more insights, we characterize the set of GE measures of risk via the pricing kernel property.

\subsection{Introduction}

Risk management is of crucial importance considering the enormous financial risk our economy is exposed to. The risks of many economic agents are regulated by various institutions. For example, if a financial trader wants to sell options, which give the buyer rights of buying or selling at a given price during a specified time horizon (or at a given time), he has to fulfil margin requirements, that is he has to deposit some cash or some other riskless and liquid instrument. An

\footnotetext{
${ }^{1}$ This chapter is based on Csóka, Herings, and Kóczy (2007b).
} 
exchange's clearing firm, which is responsible for the promises to all parties of transactions being securely completed, requires margin deposits. A measure of risk can be used to determine the margin requirement. The riskier the trader's portfolio, the more the margin requirement should be.

Other external regulators, at an international level, are the International Actuarial Association (IAA) and the International Accounting Standards Board (IASB), who determine the capital requirements for insurance companies. Similarly, the Basel Committee gives guidelines for the acceptable level of capital on banking supervision. Since a government or central bank could be a lender of last resort for these institutions, and their default could cause serious problems, they are regulated as well. As an internal regulator, a portfolio manager has to regulate the risk of its traders. In the context of a multi-division firm setting, the head-office may also set risklimits for the divisions. Internally the risk values can also be used for planning and performance evaluation. It is therefore crucial to measure risk in an appropriate way.

We will use the term portfolio when referring to a risky entity (portfolio, firm, insurance company, bank, etc.). The value of a portfolio might change due to all kinds of uncertain events. We relate risk to the probability distribution of the future value of the portfolio. For the sake of simplicity in this chapter we use discrete random variables. Our approach can be extended to the case of continuous risks and risks with unbounded support. All this requires is an analysis of competitive equilibrium in such an environment. The interested reader is referred to Chapter 10 of Duffie (2001) .

A measure of risk assigns a real number to a random variable. It is the minimal amount of cash the regulated agent has to add to his portfolio, and to invest in a zero coupon bond. Coherent measures of risk (Artzner, Delbaen, Eber, and Heath, 1999) are defined by four axioms: monotonicity, subadditivity, positive homogeneity and translation invariance. When adding two more axioms: law invariance and comonotonic additivity we get a subclass of coherent measures of risk, namely spectral measures of risk (Acerbi, 2002). Expected shortfall is a well-known spectral measure of risk (Acerbi and Tasche, 2002). For an introduction to risk measures and the above mentioned axioms see for instance Chapter 4 of Föllmer and Schied (2002).

Our approach is to model the situation at hand as an exchange economy in a general equilibrium (GE) setting, and determine which axioms are compatible with this model, and whether other axioms emerge as natural. This approach has the advantage that it recognizes the fact that the 
risk of a portfolio depends on the other assets present in the economy (the market portfolio), an insight that is generated immediately by the Capital Asset Pricing Model as developed by Sharpe (1964) and Lintner (1965). By doing so we would like to contribute to the research agenda that connects finance to GE theory, see for instance Geanakoplos and Shubik (1990), Magill and Quinzii (1996), Leroy and Werner (2001), or Jaschke and Küchler (2001). The corresponding measure of risk of a portfolio would be the amount of cash needed to sell the risk involved in the portfolio to the market. More precisely, the so-called GE measure of risk of a portfolio would be the negative of its equilibrium market price.

We prove that GE measures of risk are coherent and comonotonic additive measures of risk. However, GE measures of risk fail to satisfy law invariance, that is they are functions of not only the probability distributions of the portfolios, since they also take the regulated entity's relation to the market portfolio into account. Nevertheless we show that GE measures of risk satisfy a generalized notion of law invariance. To check on which domain spectral measures of risk are GE measures of risk, we consider a general domain for the measures of risk. We find that the corresponding domain is very small. To give more insights, we characterize GE measures of risk as the only measures of risk satisfying the property that we call the pricing kernel property.

The structure of the chapter is as follows. In Section 2.2 we discuss coherent measures of risk. In Section 2.3 spectral measures of risk are considered. Using the exchange economy model of Section 2.4 the properties of GE measures of risk are investigated in Section 2.5. In Section 2.6 we show that spectral measures of risk are GE measures of risk only under stringent conditions, and we characterize GE measures of risk via the pricing kernel property. We conclude in Section 2.7.

\subsection{Coherent Measures of Risk}

Consider a set $V \subseteq \mathbb{R}^{S}$ of realization vectors, where $S$ denotes the number of states of nature. State of nature $s$ occurs with probability $p_{s}>0$ and $\sum_{s=1}^{S} p_{s}=1$. The vector $X \in V$ represents a portfolio's (firm's, insurance company's, bank's, etc.) possible profit and loss realizations on a common chosen future time horizon, say at $t=1$. The amount $X_{s}$ is the portfolio's payoff in state of nature $s$. Negative values of $X_{s}$ correspond to losses. The inequality $Y \geq X$ means that $Y_{s} \geq X_{s}$ for all $s=1, \ldots, S$. We define $R_{+}=[0, \infty), R_{++}=(0, \infty), R_{-}=(-\infty, 0]$, 
respectively. The discrete random variable generated by $p \in \mathbb{R}_{++}^{S}$ and $X \in V$ is denoted by $\hat{X}$, that is $P\left(\hat{X}=X_{s}\right)=p_{s}$, for all $s=1, \ldots, S$.

A measure of risk is a function $\rho: V \rightarrow \mathbb{R}$ measuring the risk of a portfolio from the perspective of the present $(t=0)$. It is the minimal amount of cash the regulated agent has to add to his portfolio, and to invest in a reference instrument today, such that it ensures that the risk involved in the portfolio is acceptable to the regulator. ${ }^{2}$ We assume that the refence instrument has payoff 1 in each state of nature at $t=1$, thus its realization vector is $\mathbf{1}=(1, \ldots, 1) \in V$. The reference instrument is riskless in the "classical sense", having no uncertainty in its payoffs. It is most natural to think of it as a zero coupon bond. The price of the reference instrument, the discount factor is denoted by $\delta \in \mathbb{R}_{+}$.

We adjust the definition of coherent measures of risk to the discrete case with realization vectors on a general domain $V$ as follows.

Definition 2.1. A function $\rho: V \rightarrow \mathbb{R}$ is called a coherent measure of risk (Artzner et al., 1999) if it satisfies the following axioms.

1. Monotonicity: for all $X, Y \in V$ such that $Y \geq X$, we have $\rho(Y) \leq \rho(X)$.

2. Subadditivity: for all $X, Y \in V$ such that $X+Y \in V$, we have $\rho(X+Y) \leq \rho(X)+\rho(Y)$.

3. Positive homogeneity: for all $X \in V, h \in \mathbb{R}_{+}$such that $h X \in V$, we have $\rho(h X)=h \rho(X)$.

4. Translation invariance: for all $X \in V$ and $a \in \mathbb{R}$ such that $X+a \mathbf{1} \in V$, we have $\rho(X+a \mathbf{1})=\rho(X)-\delta a$.

The axioms are motivated as follows.

By monotonicity, if a portfolio $Y$ is always worth at least as much as $X$ (event by event), then $Y$ cannot be riskier than $X$.

Subadditivity says that if we combine two portfolios, the risk is not greater than the sum of the risks associated with each: it captures the notion of diversification. If an exchange's measure of

\footnotetext{
${ }^{2}$ The measure of risk can also be negative, meaning that a portfolio remains acceptable if a certain amount of cash is withdrawn from it.
} 
risk would fail to satisfy this property, then a trader could be better off by splitting his position, opening two accounts and decreasing the margin requirements. The same motivation applies to firms, banks, insurance companies, etc. For instance, for the case of internal capital budgeting, if the measure of risk satisfies subadditivity, then the head office can be sure that by setting risk limits to the divisions, the risk of the whole firm will be smaller than the sum of the individual risk limits. This enables the head office to decentralize the risk constraints.

Positive homogeneity requires that portfolio size should linearly influence risk.

Translation invariance ensures that the measure of risk is expressed in appropriate units. It means that investing an amount $\delta a>0$ (or $\delta a<0)$ of cash into $a$ units of reference instrument at $t=0$ leads to the payoff vector $a \mathbf{1}$ at $t=1$ and decreases (respectively: increases) the measure of risk by $\delta a$.

For further motivation of the axioms see Artzner et al., 1999.

The four axioms above seem natural, but one can easily create counterexamples showing that the rather popular Value at Risk $(\mathrm{VaR})$ is not subadditive in general and hence it is not a coherent measure of risk, see for instance Artzner et al. (1999), or Embrechts, McNeil, and Straumann (2002). However, according to Embrechts et al. (2002) VaR is coherent for elliptically distributed risks. Moreover, Ibragimov (2005) shows that VaR satisfies the coherency axioms for not extremely heavy-tailed risks with finite means and convolutions of $\alpha$-symmetric risks with $\alpha>1$, but VaR always lacks the coherency property for extremely heavy-tailed risks with infinite first moments and convolutions of $\alpha$-symmetric risks with $\alpha<1$. As a byproduct checking the relevance of the coherency axioms from a general equilibrium perspective enables us to assess this criticism on VaR as well.

\subsection{Spectral Measures of Risk}

Acerbi (2002) treats spectral measures of risk in case of discrete random variables with equiprobable outcomes, that is when $p_{1}=\cdots=p_{S}=1 / S$, as a special case. He assumes that the discount factor is 1 , and the domain is $\mathbb{R}^{S}$. In this chapter we explicitly want to use the discount factor, which for the moment is exogenously determined here as the price of the reference instrument. Since in the general equilibrium model the discount factor will be determined endogenously, we have to multiply Acerbi (2002)'s definition by $\delta$. Our model is a generalization 
also in the sense that we consider a general domain $V \subseteq \mathbb{R}^{S}$. The definition of spectral measures of risk with equiprobable outcomes is as follows.

Let us introduce the ordered statistics $X_{s: S}$ given by the ordered values of the $S$-tuple $X_{1}, \ldots, X_{S}$, that is $\left\{X_{1: S}, \ldots, X_{S: S}\right\}=\left\{X_{1}, \ldots, X_{S}\right\}$ and $X_{1: S} \leq X_{2: S} \leq \cdots \leq X_{S: S}$.

Definition 2.2. Let the outcomes be equiprobable. Consider a domain $V \subseteq \mathbb{R}^{S}$, and a vector $\phi \in \mathbb{R}^{S}$. The measure $M_{\phi}: V \rightarrow \mathbb{R}$ defined by

$$
M_{\phi}(X)=-\delta \sum_{s=1}^{S} \phi_{s} X_{s: S}
$$

is a spectral measure of risk if $\phi \in \mathbb{R}^{S}$ satisfies the conditions

1. Nonnegativity: $\phi_{s} \geq 0$ for all $s=1, \ldots, S$,

2. Normalization: $\sum_{s=1}^{S} \phi_{s}=1$,

3. Monotonicity : $\phi_{s}$ is non-increasing, that is $\phi_{s_{1}} \geq \phi_{s_{2}}$ if $s_{1}<s_{2}$ and $s_{1}, s_{2} \in\{1, \ldots, S\}$.

Spectral measures of risk are calculated as discounted weighted average losses, with nonincreasing weights, with the highest weight on the worst outcome. The weight vector $\phi$ is the so-called risk spectrum . It can be interpreted as expressing the attitude toward risk. As a special case we have the following definition.

Definition 2.3. Let $k \in\{1, \ldots, S\}$. The k-expected shortfall of the realization vector $X$ is defined by

$$
\mathrm{ES}_{k}(X)=-\delta \sum_{s=1}^{k} \frac{1}{k} X_{s: S}
$$

The $k$-expected shortfall is the discounted average of the worst $k$ outcomes. With an unrestricted domain, that is if $V=\mathbb{R}^{S}$, Acerbi (2002) has the following result.

Proposition 2.4. Assume $V=\mathbb{R}^{S}$. A measure of risk $M_{\phi}: V \rightarrow \mathbb{R}$ is coherent if and only if it is a spectral measure of risk as defined by Definition 2.2 .

Proof. Acerbi (2002), Theorem 5.3. 
Of course on a restricted domain, that is if $V \subset \mathbb{R}^{S}$, spectral measures of risk still satisfy the four coherency axioms. However, as the following three examples show, depending on $V$ one might find other measures of risk $M_{\phi}: V \rightarrow \mathbb{R}$ that are coherent but not spectral measures of risk.

Example 2.5. As a trivial example, let $V$ contain only one vector, $X$. In this case all measures of risk, i.e. all functions $\rho: V \rightarrow \mathbb{R}$ satisfy the six axioms, since there are no two vectors on which the axioms of monotonicity, subadditivity, etc. could be checked. Note that in this case spectral measures of risk are those functions $\rho: V \rightarrow \mathbb{R}$ that satisfy

$$
-\frac{\delta}{S} \sum_{s=1}^{S} X_{s: S} \leq \rho(X) \leq-\delta X_{1: S} .
$$

For all functions $\rho$ satisfying (2.3) there is $\phi \in \mathbb{R}^{S}$ such that $M_{\phi}=\rho$ and $M_{\phi}$ is a spectral measure of risk.

Interestingly, Equation (2.3) can also be justified using majorization theory. A vector $\phi \in \mathbb{R}^{S}$ is said to be majorized by a vector $\gamma \in \mathbb{R}^{S}$, written $\phi \prec \gamma$, if $\sum_{i=k}^{S} \phi_{i: S} \leq \sum_{i=k}^{S} \gamma_{i: S}, k=2, \ldots, S$ and $\sum_{i=1}^{S} \phi_{i: S}=\sum_{i=1}^{S} \gamma_{i: S}$. It is easy to see that for a risk spectrum $\phi \in \mathbb{R}^{S}$ we have that

$$
\left(\frac{1}{S}, \ldots, \frac{1}{S}\right) \prec\left(\phi_{1}, \ldots, \phi_{S}\right) \prec(1,0, \ldots, 0) .
$$

It follows from 3.H.2.c and 4.b.7 in Marshall and Olkin (1979) that $M_{\phi}$ is Schur-convex in $\phi$, that is if $\phi_{1} \prec \phi_{2}$, then for any $X \in V$ we have that $M_{\phi_{1}}(X) \leq M_{\phi_{2}}(X)$. From this observation and the relations in (2.4), Equation (2.3) follows immediately.

Example 2.6. In his proof of Theorem 5.3 (Proposition 2.4 here) Acerbi (2002) shows for the case $V=\mathbb{R}^{S}$ that if a measure of risk $M_{\phi}: V \rightarrow \mathbb{R}$ is monotone, then its risk spectrum $\phi$ should be nonnegative. If for a certain $s$ we have that $\phi_{s}<0$, then for any $X \in V$ an increase in $X_{s: S}$ gives rise to a higher measure of risk, contradicting monotonicity.

However, for many domains $V$, positivity is not required for monotonicity. Consider a domain $V=\mathbb{R}_{-}^{S-1} \times\{0\}$. In this case the best outcome of any portfolio is zero, thus the weight of the best outcome, $\phi_{S}$ can be negative, since the best outcome cannot be increased. So although $\phi$ is not nonnegative everywhere, the measure of risk remains monotone.

Example 2.7. In his proof of Theorem 5.3 (Proposition 2.4 here) Acerbi (2002) also shows for the case $V=\mathbb{R}^{S}$ that if a measure of risk $M_{\phi}: V \rightarrow \mathbb{R}$ is translation invariant, then 
its risk spectrum $\phi$ should be normalized. If $\phi$ is not normalized, then it is easy to check that translation invariance fails. It suffices to consider $M_{\phi}(X)$ and $M_{\phi}(Y)$, where $Y=X+a \mathbf{1}$, and $a \in \mathbb{R}, a \neq 0$.

For general domains $V$, normalization is not required for translation invariance. For instance, translation invariance is trivially satisfied if there are no two vectors $X$ and $Y$ such that $X=$ $Y+a \mathbf{1}$ for some $a \in \mathbb{R}, a \neq 0$. In this case translation invariance does not have bite and normalization can be omitted.

Any measure of risk $M_{\phi}: V \rightarrow \mathbb{R}$ and in particular spectral measures of risk can be shown to satisfy the axioms of law-invariance and comonotonic additivity, since they use the ordered statistics of a portfolio. The definitions of these two axioms are as follows.

Definition 2.8. A measure of risk $\rho: V \rightarrow \mathbb{R}$ is law invariant if for all $X, Y \in V$ such that for all $s=1, \ldots, S$,

$$
\operatorname{Pr}\left(\hat{X}=X_{s}\right)=\operatorname{Pr}\left(\hat{Y}=X_{s}\right)
$$

we have $\rho(X)=\rho(Y)$.

Law invariance of $\rho$ means that the measure of risk is a function of the probability distribution (law) only. Note that two random variables $\hat{X}$ and $\hat{Y}$ can be different despite the fact that they have the same probability distribution. Acerbi (2004) explains that law invariance can be thought of as the property of "being estimable from empirical data". We will point out in Section 2.5 that the portfolios' relations to the economy as a whole are also important, which will be shown to violate the law invariance axiom. For instance, if two portfolios have different covariances with the market portfolio, then the regulator may perceive their risk differently, even if their probability distribution is the same.

Another characteristic of spectral measures of risk is comonotonic additivity.

Definition 2.9. Two realization vectors $X \in \mathbb{R}^{S}$ and $Y \in \mathbb{R}^{S}$ are comonotonic if for all $s_{1}, s_{2} \in$ $\{1, \ldots, S\}$,

$$
\left(X_{s_{1}}-X_{s_{2}}\right)\left(Y_{s_{1}}-Y_{s_{2}}\right) \geq 0
$$

A map $\rho: V \rightarrow \mathbb{R}$ is comonotonic additive if given two comonotonic realization vectors $X, Y \in$ $V$ such that $X+Y \in V$ the map displays additivity, that is we have $\rho(X+Y)=\rho(X)+\rho(Y)$. 
Comonotonicity is a very strong form of dependence that two realization vectors $X$ and $Y$ can display. If two portfolios are comonotonic, then their value will always move up and down together event by event, providing no diversification at all when added to each other. The measure of risk of a portfolio consisting of two comonotonic subportfolios should therefore be equal to the sum of the measures of risk of the subportfolios. Using random variables the definition of comonotonicity is as follows.

Definition 2.10. Two random variables $\hat{X}$ and $\hat{Y}$ are comonotonic if $\hat{X}=f(\hat{Y})$ (almost surely) for some increasing function $f: \mathbb{R} \rightarrow \mathbb{R}$.

The definitions of comonotonicity for realization vectors and random variables are equivalent. For two random variables with continuous distributions $\hat{X}$ and $\hat{Y}$ the inequality in (2.5) holds for their realization vectors $X$ and $Y$ if and only if we have that the probability of concordance defined by $P\left(\left(\hat{X}-\hat{X}^{\prime}\right)\left(\hat{Y}-\hat{Y}^{\prime}\right) \geq 0\right)=1$ and consequently the probability of discordance given by $P\left(\left(\hat{X}-\hat{X}^{\prime}\right)\left(\hat{Y}-\hat{Y}^{\prime}\right) \leq 0\right)=0$, where $\hat{X}^{\prime}$ and $\hat{Y}^{\prime}$ are independent copies of $\hat{X}$ and $\hat{Y}$. In this case, one of the commonly used measures of dependence, Kendall's tau defined by

$$
\rho_{\tau}=P\left(\left(\hat{X}-\hat{X}^{\prime}\right)\left(\hat{Y}-\hat{Y}^{\prime}\right) \geq 0\right)-P\left(\left(\hat{X}-\hat{X}^{\prime}\right)\left(\hat{Y}-\hat{Y}^{\prime}\right) \leq 0\right)
$$

reaches its maximum value, that is $\rho_{\tau}=1$. From Theorem 3 in Embrechts et al. (2002) it follows that $\rho_{\tau}=1$ is equivalent with saying that $\hat{X}$ and $\hat{Y}$ are comonotonic as defined by Definition 2.10. For Kendall's tau and related measures of dependence see for instance Chapter 5 in Nelsen (1999).

Tasche (2002) shows that in the continuous, non-atomic case spectral measures of risk are the only coherent measures of risk satisfying law-invariance and comonotonic additivity. As his proof requires non-atomic probability distributions, in the discrete, equiprobable case a new proof is required for the analogous statement. The proposition requires an unrestricted domain, that is $V=\mathbb{R}^{S}$.

Proposition 2.11. Assume that the outcomes are equiprobable and $V=\mathbb{R}^{S}$. Then a measure of risk is coherent, law invariant and comonotonic additive if and only if it is a spectral measure of risk as defined by Definition 2.2 .

\section{Proof.}


$(\Leftarrow)$ The coherency part follows from Proposition 2.4. Law invariance and comonotonic additivity follows from the fact that spectral measures of risk are using the ordered statistics of a realization vector.

$(\Rightarrow)$ Take any law invariant and comonotonic additive measure of risk $\rho: \mathbb{R}^{S} \rightarrow \mathbb{R}$ satisfying the four coherency axioms. We will show that law invariance and comonotonic additivity of $\rho$ implies that it can be written in the form of Equation (2.1), that is there exists a vector $\phi \in \mathbb{R}^{S}$ such that for all $X \in \mathbb{R}^{S}$ we have that

$$
\rho(X)=M_{\phi}(X)=-\delta \sum_{s=1}^{S} \phi_{s} X_{s: S} .
$$

Law invariance of $\rho$ implies that for all $X \in \mathbb{R}^{S}$ any permutation of $X$ has the same measure of risk. A particular permutation is the ordered statistics vector of $X,\left[X_{s: S}\right]$. Using the notation $\bar{X}=\left\{X \in \mathbb{R}^{S} \mid X_{1} \leq X_{2} \leq \cdots \leq X_{S}\right\}$ for the space of ordered statistics, we have that for all $X \in \mathbb{R}^{S}$ the measure of risk $\rho$ can be written as a function $f: \bar{X} \rightarrow \mathbb{R}$ of the ordered statistics vector,

$$
\rho(X)=f\left(\left[X_{s: S}\right]\right)
$$

Comonotonic additivity of $\rho$ implies that $f$ is linear on $\bar{X}$. Choosing $S$ independent vectors in $\bar{X}$ implies that $f$ is additive separable with constant weight functions $\bar{\phi}_{1}, \ldots, \bar{\phi}_{S} \in \mathbb{R}$, that is

$$
f\left(\left[X_{s: S}\right]\right)=\bar{\phi}_{1} X_{1: S}+\cdots+\bar{\phi}_{S} X_{S: S}
$$

Using $\phi_{s}=-\frac{\bar{\phi}_{s}}{\delta}, s=1, \ldots, S$ we have that Equation (2.7) is satisfied. Proposition 2.4 implies that $\rho$ should be a spectral measure of risk.

It is cumbersome to generalize the definition of spectral measures of risk to the case in which the outcomes are not equiprobable. Consider the following example.

Example 2.12. In Table 2.1 we have 4 states of nature with equal probability of occurrence. Portfolios $X$ and $Y$, their ordered statistics, and the risk spectrum $\phi$ are also given. A straightforward calculation gives $M_{\phi}(X)=0$, and $M_{\phi}(Y)=3.5 \delta$.

Since the portfolios $X$ and $Y$ have the same outcome in states of nature 2, 3 and 4, we can merge those states. This operation leads to Table 2.2, with portfolios $X^{\prime}$ and $Y^{\prime}$, where the generalized discrete risk spectrum $\phi^{g}$ is given by $\phi_{1}^{g}=\phi_{1}$ and $\phi_{2}^{g}=\phi_{2}+\phi_{3}+\phi_{4}$. 


\begin{tabular}{c|cccccc}
$s$ & $p_{s}$ & $X_{s}$ & $X_{s: S}$ & $Y_{s}$ & $Y_{s: S}$ & $\phi_{s}$ \\
\hline 1 & 0.25 & -3 & -3 & 1 & -4 & 0.4 \\
2 & 0.25 & 2 & 2 & -4 & -4 & 0.3 \\
3 & 0.25 & 2 & 2 & -4 & -4 & 0.2 \\
4 & 0.25 & 2 & 2 & -4 & 1 & 0.1
\end{tabular}

Table 2.1: Spectral measures of risk with equiprobable outcomes.

\begin{tabular}{c|cccccc}
$s$ & $p_{s}^{\prime}$ & $X_{s}^{\prime}$ & $X_{s: S}^{\prime}$ & $Y_{s}^{\prime}$ & $Y_{s: S}^{\prime}$ & $\phi_{s}^{g}$ \\
\hline 1 & 0.25 & -3 & -3 & 1 & -4 & 0.4 \\
2 & 0.75 & 2 & 2 & -4 & 1 & 0.6
\end{tabular}

Table 2.2: Spectral measures of risk with not equiprobable outcomes.

By defining $M_{\phi^{g}}(X)=-\delta \sum_{s=1}^{S} \phi_{s}^{g} X_{s: S}$ we have that $M_{\phi}(X)=M_{\phi^{g}}\left(X^{\prime}\right)=0$, but $M_{\phi}(Y)=$ $3.5 \delta$, whereas $M_{\phi^{g}}\left(Y^{\prime}\right)=\delta$. To avoid this problem, within the discrete setting a different $\phi^{g}$ should be specified for all the orderings of the portfolio vectors. This would rather complicate the analysis and would not add much value since with splitting up the states and increasing their number any discrete distribution can be arbitrarily closely approximated by equiprobable states. Thus we will use the discrete, equiprobable version of spectral measures of risk as defined in Definition 2.2 in the sequel.

\subsection{An Exchange Economy Model}

Next we discuss the four axioms underlying coherent measures of risk and the additional two axioms of spectral measures of risk from a general equilibrium perspective. We do this by checking the validity of the six axioms on the natural measure of risk derived from an exchange economy model, which we call the General Equilibrium $(G E)$ measure of risk. The GE measure of risk of a portfolio is the amount of cash needed to sell the risk involved in the portfolio to the market. More precisely, it is the minimal amount of cash needed to add to the portfolio, such that its market price becomes nonnegative. It is easy to see that the GE measure of risk of a portfolio is the negative of its equilibrium market price.

To specify the GE measure of risk we consider an exchange economy model with two time periods $(t=0, t=1)$ and uncertainty concerning the state of nature in period $t=1$. We assume that for each state $s$ in the set $\{1, \ldots, S\}$ its probability of occurrence is objectively known, 
$p_{s}=1 / S .^{3}$ Period $t=0$ is identified with state $s=0$. There is a unique nondurable commodity (income) in each state of nature $s=0, \ldots, S$.

The portfolios (firms, insurance companies, banks, etc.) are represented as exogenously given realization vectors in $\mathbb{R}^{S}$. Their payoff is a profit or loss in state of nature $s=1, \ldots, S$.

To simplify the analysis, we assume that the economy can be modeled by means of a representative agent. However, it is not difficult to extend the model to the case with heterogeneous agents. We assume that the consumption set of the agent is $\mathbb{R}$ in all states of nature. His consumption stream is denoted by $c=\left(c_{0}, \ldots, c_{S}\right) \in \mathbb{R}^{S+1}$.

The agent's preferences are represented by a von Neumann-Morgenstern utility function $u$ : $\mathbb{R}^{S+1} \rightarrow \mathbb{R}$, given by

$$
u\left(c_{0}, \ldots, c_{S}\right)=v_{0}\left(c_{0}\right)+\bar{\delta} \sum_{s=1}^{S} p_{s} v\left(c_{s}\right),
$$

where $v_{0}: \mathbb{R} \rightarrow \mathbb{R}$ and $v: \mathbb{R} \rightarrow \mathbb{R}$ are elementary (Bernoulli) utility functions in state 0 and states $s=1, \ldots, S$ respectively; the scalar $\bar{\delta} \in \mathbb{R}_{++}$is the subjective discount factor of the agent. Moreover, the representative agent is characterized by his (aggregate) endowment $\omega=\left(\omega_{0}, \ldots, \omega_{S}\right) \in \mathbb{R}^{S+1}$. The endowment expresses the agent's consumption possibilities without trade, depending on the realization of the state of nature. To distinguish between the sure zero-period endowment and the random first-period endowment, we define $\widetilde{\omega}=\left(\omega_{1}, \ldots, \omega_{S}\right)$.

We assume that income transfers across all states are possible, that is markets are complete. Without loss of generality we assume that there are $S$ contingent contracts .

Definition 2.13. A contingent contract for state $s(s=1, \ldots, S)$ is a promise to deliver one unit of income in state $s$ and nothing otherwise. The price of the contingent contract, the state price, expressed in units of period 0 income, is denoted by $\pi_{s}$ for $s=1, \ldots, S$. Furthermore, $\pi_{0}=1$.

The agent can sell his endowment $\omega$, from which he can purchase on the contingent markets any consumption stream $c$ satisfying the budget inequality

$$
\sum_{s=0}^{S} \pi_{s} c_{s} \leq \sum_{s=0}^{S} \pi_{s} \omega_{s}
$$

The agent's utility function and his endowment define an economy $\mathcal{E}=(u, \omega)$. The equilibrium state prices are determined by the notion of competitive equilibrium.

\footnotetext{
${ }^{3}$ We shall only employ the equiprobable assumption when comparing GE measures of risk to spectral measures of risk.
} 
Definition 2.14. A competitive equilibrium for an economy $\mathcal{E}=(u, \omega)$ is a consumption vector $c^{*}=\left(c_{0}^{*}, \ldots, c_{S}^{*}\right)$ and state price vector $\pi^{*}=\left(\pi_{1}^{*}, \ldots, \pi_{S}^{*}\right)$ that satisfy the following conditions:

1. $c^{*} \in \arg \max _{c} u\left(c_{0}, \ldots, c_{S}\right)$ s.t. $\sum_{s=0}^{S} \pi_{s}^{*} c_{s} \leq \sum_{s=0}^{S} \pi_{s}^{*} \omega_{s}$,

2. $c^{*}=\omega$.

We present two sufficient conditions for the existence of an equilibrium with nonnegative state prices.

Proposition 2.15. Consider an economy $\mathcal{E}=(u, \omega)$. If the elementary utility function $v_{0}: \mathbb{R} \rightarrow$ $\mathbb{R}$ is strictly monotonic and concave and $v: \mathbb{R} \rightarrow \mathbb{R}$ is monotone and concave, then a competitive equilibrium $\left(c^{*}, \pi^{*}\right)$ exists. Moreover, $\pi_{s}^{*} \geq 0$ for $s=1, \ldots, S$.

Let $\mathcal{U}$ denote the set of utility functions $u$ in which the elementary utility functions $v_{0}: \mathbb{R} \rightarrow \mathbb{R}$ and $v: \mathbb{R} \rightarrow \mathbb{R}$ are twice differentiable, $v_{0}^{\prime}>0, v_{0}^{\prime \prime} \leq 0$ and $v^{\prime} \geq 0, v^{\prime \prime} \leq 0$.

Proposition 2.16. Consider an economy $\mathcal{E}=(u, \omega)$. If $u \in \mathcal{U}$, then the competitive equilibrium $\left(c^{*}, \pi^{*}\right)$ is unique. Moreover,

$$
\pi_{s}^{*}=\frac{\frac{\partial u(\omega)}{\partial c_{s}}}{\frac{\partial u(\omega)}{\partial c_{0}}}=\frac{\bar{\delta} p_{s} v^{\prime}\left(\omega_{s}\right)}{v_{0}^{\prime}\left(\omega_{0}\right)} \geq 0, \quad s=1, \ldots, S .
$$

The proofs of Proposition 2.15 and Proposition 2.16 are standard results in microeconomic theory, see for instance Mas-Colell, Whinston, and Green (1995).

Under the assumptions of Proposition 2.15 or Proposition 2.16 one can define the equilibrium price of any portfolio $Z \in \mathbb{R}^{S}$.

Definition 2.17. If $\left(c^{*}, \pi^{*}\right)$ is a competitive equilibrium of an economy $\mathcal{E}=(u, \omega)$, then the equilibrium price, $q_{Z}^{*}$ of the portfolio $Z \in \mathbb{R}^{S}$ is given by $q_{Z}^{*}=\sum_{s=1}^{S} \pi_{s}^{*} Z_{s}$.

Note that under the assumption of Proposition 2.16 the equilibrium price of the portfolio $Z \in \mathbb{R}^{S}$ is uniquely given by

$$
q_{Z}^{*}=\sum_{s=1}^{S} \pi_{s}^{*} Z_{s}=\bar{\delta} \frac{\sum_{s=1}^{S} p_{s} v^{\prime}\left(\omega_{s}\right) Z_{s}}{v_{0}^{\prime}\left(\omega_{0}\right)} .
$$

As an important special case of Equation (2.12), the discount factor becomes

$$
\delta=q_{\mathbf{1}}^{*}=\sum_{s=1}^{S} \pi_{s}^{*} 1=\bar{\delta} \frac{\sum_{s=1}^{S} p_{s} v^{\prime}\left(\omega_{s}\right)}{v_{0}^{\prime}\left(\omega_{0}\right)} .
$$

Note that the discount factor is endogenously determined in the exchange economy model. 


\subsection{General Equilibrium Measures of Risk}

Now we can define the GE measure of risk of a portfolio as the negative of its equilibrium market price.

Definition 2.18. Consider an economy $\mathcal{E}=(u, \omega)$ with competitive equilibrium $\left(c^{*}, \pi^{*}\right)$ and a domain $V$. The GE measure of risk is the map $\rho_{u, \omega}: V \rightarrow \mathbb{R}$, where $\rho_{u, \omega}(Z)=-q_{Z}^{*}$ for any $Z \in V$.

Note that Definition 2.17 and Definition 2.18 imply that for any $Z \in V$

$$
\rho_{u, \omega}(Z)=-q_{Z}^{*}=-\sum_{s=1}^{S} \pi_{s}^{*} Z_{s}
$$

Notice that $\rho_{u, \omega}$ is a linear function.

Example 2.19. Consider an economy $\mathcal{E}=(u, \omega)$ and a domain $V$. Let $v_{0}\left(c_{0}\right)=c_{0}-\frac{1}{2} \alpha c_{0}^{2}$ and $v\left(c_{s}\right)=c_{s}-\frac{1}{2} \alpha c_{s}^{2}$ for $s=1, \ldots, S$, where $\alpha \in \mathbb{R}_{++}$such that $1-\alpha \omega_{s}>0, s=0, \ldots, S$, that is the elementary utility functions are quadratic and increasing at $c^{*}=\omega$. Since $u \in \mathcal{U}$, using Equations (2.11) and (2.14) the GE measure of risk of $Z \in V$ is given by

$$
\rho_{u, \omega}(Z)=-q_{Z}^{*}=-\sum_{s=1}^{S} \pi_{s}^{*} Z_{s}=-\bar{\delta} \frac{\sum_{s=1}^{S} p_{s} v^{\prime}\left(\omega_{s}\right) Z_{s}}{v_{0}^{\prime}\left(\omega_{0}\right)}=-\bar{\delta} \frac{\sum_{s=1}^{S} p_{s}\left(1-\alpha \omega_{s}\right) Z_{s}}{\left(1-\alpha \omega_{0}\right)} .
$$

One can also look at the stochastic part of the aggregate endowment $\widetilde{\omega}$ as representing the market portfolio, since it captures the aggregate uncertainties. Let us denote the total return of any portfolio $\mathrm{Z}$ by $r_{Z}$, where the total return is the payoff vector divided by the price: $r_{Z}=Z / q_{Z}^{*}$. It is well known (see for instance Geanakoplos and Shubik (1990)) that with a quadratic utility function the CAPM formula holds. It relates the total return of any portfolio to the total return of the market portfolio as follows.

$$
E\left(r_{Z}\right)=r_{\mathbf{1}}+\beta_{Z}\left(E\left(r_{\widetilde{\omega}}\right)-r_{\mathbf{1}}\right), \text { where } \beta_{Z}=\frac{\operatorname{Cov}\left(r_{Z}, r_{\widetilde{\omega}}\right)}{\operatorname{Var}\left(r_{\tilde{\omega}}\right)}
$$

Note that the total return of the reference instrument is given by $r_{1}=1 / \delta$. From $q_{Z}^{*}=$ $E(Z) / E\left(r_{Z}\right)$ and Equation (2.16) it follows that

$$
\rho_{u, \omega}(Z)=-q_{Z}^{*}=-\frac{E(Z)}{r_{\mathbf{1}}+\beta_{Z}\left(E\left(r_{\widetilde{\omega}}\right)-r_{\mathbf{1}}\right)} .
$$


Thus if the elementary utility functions are quadratic, then the GE measure of risk of portfolio $Z$ is its discounted expected loss $-E(Z) / r_{\mathbf{1}}=-\delta E(Z)$, corrected with its risk relation to the aggregate endowment.

Using the differentiability assumption let us denote the set of GE measures of risk on $V$ by $G_{V}$, where

$$
G_{V}=\left\{\rho_{u, \omega}: V \rightarrow \mathbb{R} \mid u \in \mathcal{U}, \omega \in \mathbb{R}^{S+1}\right\}
$$

Let us discuss some properties of $G_{V}$.

Proposition 2.20. Any $\rho_{u, \omega} \in G_{V}$ is a coherent measure of risk.

Proof. By Proposition 2.16, $\pi_{s}^{*} \geq 0$ for $s=1, \ldots, S$. Using this fact and the linearity of $\rho_{u, \omega}$ monotonicity, subadditivity and positive homogeneity follows immediately. For translation invariance note that $\delta=\sum_{s=1}^{S} \pi_{s}^{*}$ by Equation (2.13). Then for all $\mathrm{Z} \in V$, for all $a \in \mathbb{R}$ such that $Z+a \mathbf{1} \in V$, we have

$$
\begin{aligned}
\rho_{u, \omega}(Z+\mathbf{1} a) & =-q_{Z+\mathbf{1} a}^{*}=-\sum_{s=1}^{S} \pi_{s}^{*}\left(Z_{s}+a\right)=-\sum_{s=1}^{S} \pi_{s}^{*}\left(Z_{s}\right)-\sum_{s=1}^{S} \pi_{s}^{*} a= \\
& =-q_{Z}^{*}-\delta a=\rho_{u, \omega}(Z)-\delta a .
\end{aligned}
$$

Note that GE measures of risk are coherent measures of risk under the assumptions of Proposition 2.15 as well. Interestingly, in Proposition 6, Jaschke and Küchler (2001) presents a similar result for a family of normalized, non-negative price systems.

As GE measures of risk are coherent and their linearity also implies comonotonic additivity, so far five out of six axioms of spectral measures of risk are satisfied by them. The sixth one is law invariance. The following example shows that GE measures of risk are not law invariant.

Example 2.21. As a special case of Example 2.19 consider 2 states of nature at $t=1$ with equal probability of occurrence. Let $\bar{\delta}=1$ and $\alpha=1$. Two portfolios, $X^{1}$ and $X^{2}$, the aggregate endowment, and the state prices calculated by Equation (2.15) are given in Table 2.3 below.

Let us assume that $X^{1}, X^{2} \in V$. It is easy to see that $\hat{X}^{1}$ and $\hat{X}^{2}$ have the same probability distribution. However, $\rho_{u, \omega}\left(X^{1}\right)=0.9 \neq 1.05=\rho_{u, \omega}\left(X^{2}\right)$, so law invariance fails. 


\begin{tabular}{c|ccccc}
$s$ & $p_{s}$ & $X^{1}$ & $X^{2}$ & $\omega$ & $\pi_{s}^{*}$ \\
\hline 0 & - & - & - & 0 & 1 \\
1 & 0.5 & -1 & -2 & 0.2 & 0.4 \\
2 & 0.5 & -2 & -1 & 0.5 & 0.25
\end{tabular}

Table 2.3: A GE measure of risk which is not law invariant.

Note that the two portfolios in Table 2.3 are related differently to the economy. Portfolio $X^{1}$ is larger when $\omega$ is smaller, whereas $X^{2}$ is smaller when $\omega$ is smaller. Although the two portfolios have the same mean, $X^{1}$ is a better hedge against the aggregate uncertainty. That is why the GE measure of risk of $X^{1}$ is smaller than the GE measure of risk of $X^{2}$.

One can easily calculate that in Example $2.21 r_{\mathbf{1}}=1.5385$, and $E\left(r_{\widetilde{\omega}}\right)=1.7073$. Using those numbers we can apply Equation (2.17) and calculate the GE measures of risk of $X^{1}$ and $X^{2}$ through the CAPM formula as it is given in Table 2.4. Note that $\beta_{X^{1}}$ is positive, whereas $\beta_{X^{2}}$ is negative.

\begin{tabular}{c|cc} 
& $X^{1}$ & $X^{2}$ \\
\hline$E\left(X^{i}\right)$ & -1.5 & -1.5 \\
$\beta_{X^{i}}$ & 0.7593 & -0.6508 \\
$\rho_{u, \omega}\left(X^{i}\right)$ & 0.9 & 1.05
\end{tabular}

Table 2.4: A GE measure of risk and the CAPM formula.

Most of the GE measures of risk are not law invariant. In Example 2.21 all that is needed is that the state prices are different, which is the case in a generic economy. The failure of law invariance tells us that the stochastic part of the aggregate endowment (or market portfolio, in terms of CAPM) should be taken into account, when calculating the risk of a portfolio. Nevertheless one can generalize law invariance in such a way that GE measures of risk satisfy them. The point is to look at the joint distribution of a portfolio and the stochastic part of the aggregate endowment.

Definition 2.22. A measure of risk $\rho: V \rightarrow \mathbb{R}$ is generalized law invariant if for all $X, Y \in V$ such that for all $s=1, \ldots, S$

$$
\operatorname{Pr}\left(\hat{X}=X_{s} \text { and } \hat{\omega}=\omega_{s}\right)=\operatorname{Pr}\left(\hat{Y}=X_{s} \text { and } \hat{\omega}=\omega_{s}\right)
$$

we have $\rho(X)=\rho(Y)$.

Proposition 2.23. GE measures of risk are generalized law invariant. 
Proof. It is easy to see from Equation (2.12) and Definition 2.18 that any $\rho_{u, \omega} \in G_{V}$ is generalized law invariant.

\subsection{Connections: Spectral and GE Measures of Risk}

In this section we show the conditions under which spectral measures of risk as defined in Definition 2.2 are GE measures of risk as defined in Definition 2.18. Then we characterize GE measures of risk.

Definition 2.24. A vector $\widetilde{\omega} \in \mathbb{R}^{S}$ and a set $V \subseteq \mathbb{R}^{S}$ are comonotonic if $\widetilde{\omega}$ and $Z$ are comonotonic for every $Z \in V$.

Proposition 2.25 says that all the spectral measures of risk are GE measures of risk if and only if their domain is comonotonic with the stochastic part of the aggregate endowment, that is all the vectors in $V$ are comonotonic with $\widetilde{\omega}$, their values go up and down together event by event.

Proposition 2.25. Assume $\omega$ is given and it has different outcomes in states $s=1, \ldots, S$. Moreover, let $V$ contain $S$ linearly independent vectors which are comonotonic with $\widetilde{\omega}$. Then for every $M_{\phi}$ there exists a GE measure of risk $\rho_{u, \omega} \in G_{V}$ such that

$$
M_{\phi}(Z)=\rho_{u, \omega}(Z) \text { for all } Z \in V
$$

if and only if $\widetilde{\omega}$ and $V$ are comonotonic.

Proof. Since $\widetilde{\omega}$ has different outcomes in each state of nature by assumption, without loss of generality we can assume that $\omega_{1}<\omega_{2}<\cdots<\omega_{S}$.

$(\Leftarrow)$ We show that comonotonicity is sufficient. Take any spectral measure of risk, $M_{\phi}$ and any $Z \in V$. Since $\widetilde{\omega}$ and $V$ are comonotonic, $\widetilde{\omega}$ and $Z$ are also comonotonic. Let us search for a GE measure of risk $\rho_{u, \omega} \in G_{V}$ satisfying the equation $M_{\phi}(Z)=\rho_{u, \omega}(Z)$. Since $\omega$ is given, the only freedom is in choosing $u \in \mathcal{U}$. Using the definitions the requirement is

$$
M_{\phi}(Z)=-\delta \sum_{s=1}^{S} \phi_{s} Z_{s: S}=-\bar{\delta} \sum_{s=1}^{S} \frac{p_{s} v^{\prime}\left(\omega_{s}\right) Z_{s}}{v_{0}^{\prime}\left(\omega_{0}\right)}=\rho_{u, \omega}(Z) .
$$

From Equation (2.13) the discount factor is determined as

$$
\delta=\bar{\delta} \frac{\sum_{s=1}^{S} p_{s} v^{\prime}\left(\omega_{s}\right)}{v_{0}^{\prime}\left(\omega_{0}\right)} .
$$


Using Equation (2.19) and the assumption that $p_{1}=\cdots=p_{S}=\frac{1}{S}$, Equation (2.18) reads as

$$
-\bar{\delta} \frac{\sum_{s=1}^{S} \frac{1}{S} v^{\prime}\left(\omega_{s}\right)}{v_{0}^{\prime}\left(\omega_{0}\right)} \sum_{s=1}^{S} \phi_{s} Z_{s: S}=-\bar{\delta} \sum_{s=1}^{S} \frac{\frac{1}{S} v^{\prime}\left(\omega_{s}\right) Z_{s}}{v_{0}^{\prime}\left(\omega_{0}\right)} .
$$

After simplifying and rearranging, Equation (2.20) leads to

$$
\sum_{s=1}^{S} \phi_{s} Z_{s: S}=\frac{\sum_{s=1}^{S} v^{\prime}\left(\omega_{s}\right) Z_{s}}{\sum_{s=1}^{S} v^{\prime}\left(\omega_{s}\right)}
$$

In Equation (2.21) two weighted averages of $Z$ should coincide. Since $\widetilde{\omega}$ and $Z$ are comonotonic, a larger $\widetilde{\omega}$ implies a larger or equal $Z$. Thus the ordered and the non-ordered statistics of $Z$ coincide, that is $Z_{s: S}=Z_{s}$ for all $s=1, \ldots, S$. The weights on the left-hand side are by definition normalized, nonnegative, and non-increasing. The weights on the right-hand side are also normalized, and it is easy to see that $v$ can be chosen monotone and concave to obtain the same weights.

$(\Rightarrow)$ We show that comonotonicity is necessary.

By assumption $V$ contains $S$ linearly independent vectors which are comonotonic with $\widetilde{\omega}$. Let us denote them by $Z^{1}, \ldots, Z^{\bar{S}} \in V$, where $\bar{S}=S$. Since for all $\bar{s}=1, \ldots, \bar{S}$ we have that $Z^{\bar{s}}$ and $\widetilde{\omega}$ and comonotonic, we have that

$$
Z_{s: S}^{\bar{s}}=Z_{s}^{\bar{s}} \text { for all } s=1, \ldots, S
$$

that is the ordered and the non-ordered statistics of $Z^{\bar{s}}$ coincide. Since the proposition should hold for any given $M_{\phi}$, let us take a spectral measure of risk with different $\phi_{s}$ in each state of nature. We show indirectly that if $\widetilde{\omega}$ and $V$ are not comonotonic, then there is no GE measure of risk $\rho_{u, \omega} \in G_{V}$ such that $M_{\phi}(Z)$ yields the same number as $\rho_{u, \omega}(Z)$ for all $Z \in V$.

If $M_{\phi}\left(Z^{\bar{s}}\right)$ yields the same number as $\rho_{u, \omega}\left(Z^{\bar{s}}\right)$ for $\bar{s}=1, \ldots, \bar{S}$, then using the logic of Equation (2.21) and the result in Equation (2.22) the following equations are satisfied:

$$
\sum_{s=1}^{S} \phi_{s} Z_{s}^{\bar{s}}=\frac{\sum_{s=1}^{S} v^{\prime}\left(\omega_{s}\right) Z_{s}^{\bar{s}}}{\sum_{s=1}^{S} v^{\prime}\left(\omega_{s}\right)} \text { for } \bar{s}=1, \ldots, \bar{S} .
$$

As the equations in (2.23) are homogeneous, we can assume that $\sum_{s=1}^{S} v^{\prime}\left(\omega_{s}\right)=1$. Since the vectors $Z^{1}, \ldots, Z^{\bar{S}}$ are linearly independent, the equations in (2.23) determine that

$$
v^{\prime}\left(\omega_{s}\right)=\phi_{s} \text { for } s=1, \ldots, S
$$


Now take any $Z \in V$ which is not comonotonic with $\widetilde{\omega}$ (such $Z$ exists since $\widetilde{\omega}$ and $V$ are not comonotonic). It follows from the definition of comonotonicity that there exist states of nature $s_{1}$ and $s_{2}$ in which

$$
\omega_{s_{1}}<\omega_{s_{2}} \text { but } Z_{s_{1}}>Z_{s_{2}} \text {. }
$$

Using Equation (2.21) and Equation (2.24) we get that $M_{\phi}(Z)=\rho_{u, \omega}(Z)$ if and only if

$$
\sum_{s=1}^{S} \phi_{s} Z_{s: S}=\sum_{s=1}^{S} \phi_{s} Z_{s}
$$

Both sides of Equation (2.26) are weighted averages of $Z$. The left-hand side assigns strictly decreasing weights to the ordered statistics of $Z$. The right-hand side assigns the same weights but due to the observation in (2.25) at least one higher outcome of $Z$ gets a higher weight, leading to

$$
\sum_{s=1}^{S} \phi_{s} Z_{s: S}<\sum_{s=1}^{S} \phi_{s} Z_{s} .
$$

We have a contradiction.

Note that we had to assume in Proposition 2.25 that $\widetilde{\omega}$ has different outcomes in each state of nature. In a generic economy this assumption is satisfied. The second assumption was that $V$ contains $S$ linearly independent vectors which are comonotonic with $\widetilde{\omega}$. It is easy to check that this assumption is satisfied if $V$ contains all the vectors which are comonotonic with $\widetilde{\omega}$ or if $V$ contains a convex cone generated by $\widetilde{\omega}$ and $S-1$ linearly independent vectors. However, if we only require that $V$ contains $\widetilde{\omega}$, then Proposition 2.25 cannot be proven as the following example shows.

Example 2.26. Suppose $V=\{\widetilde{\omega}, Z\}$, where $\widetilde{\omega}$ and $Z$ are given in Table 2.5 below.

\begin{tabular}{c|cc}
$\mathrm{s}$ & $\widetilde{\omega}_{s}$ & $Z_{s}$ \\
\hline 1 & 1 & -2 \\
2 & 2 & -1 \\
3 & 3 & -1.25
\end{tabular}

Table 2.5: $Z$ is not comonotonic with $\widetilde{\omega}$. 
Note that the vector $\widetilde{\omega}$ and $V$ are not comonotonic in Table 2.5, since $Z \in V$ is not comonotonic with $\widetilde{\omega}$. We show that in this example for every $M_{\phi}$ there exists a GE measure of risk $\rho_{u, \omega} \in G_{V}$ such that $M_{\phi}(Z)=\rho_{u, \omega}(Z)$ for all $Z \in V$, although $\widetilde{\omega}$ and $V$ are not comonotonic. Using the normalized version of Equation (2.21) we have that $M_{\phi}$ and $\rho_{u, \omega}$ lead to the same number for $\widetilde{\omega}$ if

$$
\begin{aligned}
& \phi_{1}+2 \phi_{2}+3 \phi_{3}=v^{\prime}\left(\widetilde{\omega}_{1}\right)+2 v^{\prime}\left(\widetilde{\omega}_{2}\right)+3 v^{\prime}\left(\widetilde{\omega}_{3}\right), \text { and for } Z \text { if } \\
& -2 \phi_{1}-1.25 \phi_{2}-\phi_{3}=-2 v^{\prime}\left(\widetilde{\omega}_{1}\right)-v^{\prime}\left(\widetilde{\omega}_{2}\right)-1.25 v^{\prime}\left(\widetilde{\omega}_{3}\right) .
\end{aligned}
$$

Moreover, we know that

$$
\begin{aligned}
& \phi_{1}+\phi_{2}+\phi_{3}=v^{\prime}\left(\widetilde{\omega}_{1}\right)+v^{\prime}\left(\widetilde{\omega}_{2}\right)+v^{\prime}\left(\widetilde{\omega}_{3}\right)=1, \text { and by definition } \\
& \phi_{1} \geq \phi_{2} \geq \phi_{3} .
\end{aligned}
$$

Solving the Equations (2.28), (2.29) and (2.30) leads to the unique solution

$$
\begin{aligned}
& v^{\prime}\left(\widetilde{\omega}_{1}\right)=\phi_{1}+\frac{1}{5} \phi_{2}-\frac{1}{5} \phi_{3}, \\
& v^{\prime}\left(\widetilde{\omega}_{2}\right)=\frac{3}{5} \phi_{2}+\frac{2}{5} \phi_{3}, \\
& v^{\prime}\left(\widetilde{\omega}_{3}\right)=\frac{1}{5} \phi_{2}+\frac{4}{5} \phi_{3} .
\end{aligned}
$$

Since $v$ is assumed to be concave,

$$
v^{\prime}\left(\widetilde{\omega}_{1}\right) \geq v^{\prime}\left(\widetilde{\omega}_{2}\right) \geq v^{\prime}\left(\widetilde{\omega}_{3}\right) \text { should hold. }
$$

From Equation (2.31) we have that $\phi_{2} \geq \phi_{3}$. This observation implies with Equation (2.32) that $v^{\prime}\left(\widetilde{\omega}_{1}\right) \geq \phi_{1}$; with Equations (2.33), (2.34) that $v^{\prime}\left(\widetilde{\omega}_{2}\right) \geq v^{\prime}\left(\widetilde{\omega}_{3}\right)$; and with Equation (2.33) that $v^{\prime}\left(\widetilde{\omega}_{2}\right) \leq \phi_{2}$. Since $\phi_{1} \geq \phi_{2}$ from Equation (2.31) the inequalities in Equation (2.35) are clearly satisfied.

The strong requirement in Proposition 2.25 is that $\widetilde{w}$ and $V$ should be comonotonic to represent all the spectral measures of risk by GE measures of risk. In this case all the vectors in $V$ are dependent in the strongest form on the stochastic part of the aggregate endowment, their value should always go up and down together event by event. The larger the number of states $S$, the more stringent this assumption is.

We can characterize the set of GE measures of risk as follows. 
Definition 2.27. A measure of risk $\rho: V \rightarrow \mathbb{R}$ satisfies the pricing kernel property on $V$ if there exists a vector $\gamma \in \mathbb{R}_{+}^{S}$ such that for all $Z \in V$ we have that

$$
\rho(Z)=-\sum_{s=1}^{S} p_{s} \gamma_{s} Z_{s}
$$

The vector $\gamma$ is interpreted as the pricing kernel, and the measure of risk of portfolio $Z$ is the negative of its price, induced by the pricing kernel. Let us denote by $P_{V}$ the set of risk measures satisfying the pricing kernel property on $V$. Then we have the following proposition.

Proposition 2.28. The set of GE measures of risk on $V$ coincides with the set of risk measures satisfying the pricing kernel property on $V$, thus $G_{V}=P_{V}$.

\section{Proof.}

$G_{V} \subseteq P_{V}$

For any $\rho_{u, \omega} \in G_{V}$, by Equation (2.14) we have that

$$
\rho_{u, \omega}(Z)=-\sum_{s=1}^{S} \pi_{s}^{*} Z_{s}
$$

By Proposition 2.16, $\pi^{*} \in \mathbb{R}_{+}^{S}$. Using the notation $\gamma_{s}=\pi_{s}^{*} / p_{s}, s=1, \ldots, S$, Equation (2.37) can be rewritten as

$$
\rho_{u, \omega}(Z)=-\sum_{s=1}^{S} p_{s} \gamma_{s} Z_{s}, \gamma \in \mathbb{R}_{+}^{S}
$$

thus $\rho_{u, \omega}$ satisfies the pricing kernel property.

$G_{V} \supseteq P_{V}$

Let us take any measure of risk $\bar{\rho} \in P_{V}$ satisfying the pricing kernel property on $V$. By definition there exists a vector $\gamma \in \mathbb{R}_{+}^{S}$ such that for all $Z \in V$ we have that

$$
\bar{\rho}(Z)=-\sum_{s=1}^{S} p_{s} \gamma_{s} Z_{s}
$$

We show that there exist a quadratic utility function $u$ and endowment $\omega \in \mathbb{R}^{S+1}$ such that for all $Z \in V$ we have that $\bar{\rho}(Z)=\rho_{u, \omega}(Z)$. Let $v_{0}\left(c_{0}\right)=c_{0}-\frac{1}{2} \alpha c_{0}^{2}$ and $v\left(c_{s}\right)=c_{s}-\frac{1}{2} \alpha c_{s}^{2}$ for $s=1, \ldots, S$. In equilibrium $c^{*}=\omega$. We know from Equation (2.15) that

$$
\rho_{u, \omega}(Z)=-\bar{\delta} \frac{\sum_{s=1}^{S} p_{s} v^{\prime}\left(\omega_{s}\right) Z_{s}}{v_{0}^{\prime}\left(\omega_{0}\right)}=-\bar{\delta} \frac{\sum_{s=1}^{S} p_{s}\left(1-\alpha \omega_{s}\right) Z_{s}}{\left(1-\alpha \omega_{0}\right)} .
$$


Let $\alpha=1, \bar{\delta}=1$ and $\omega_{0}=0$. Then Equation (2.40) simplifies to

$$
\rho_{u, \omega}(Z)=-\sum_{s=1}^{S} p_{s}\left(1-\omega_{s}\right) Z_{s} .
$$

By setting $\omega_{s}$ such that $\gamma_{s}=\left(1-\omega_{s}\right), s=1, \ldots, S$, Equation (2.39) and Equation (2.41) define the same measure of risk. As $v_{0}^{\prime}=1$ and $v_{s}^{\prime}=\gamma_{s}, s=1, \ldots, S$, the monotonicity requirements of the elementary utility functions are satisfied.

Thus GE measures of risk are the only measures of risk satisfying the pricing kernel property. They are linear functions induced by $S$ nonnegative parameters $\left(\gamma_{1}, \ldots, \gamma_{S}\right)$. On the other hand spectral measures of risk are piecewise linear functions, also induced by $S$ parameters $\left(\phi_{1}, \ldots, \phi_{S}\right)$, which are applied to the ordered statistics of the portfolio's payoff vector. Thus, although the cardinalities of their sets are the same, under some technical assumptions spectral measures of risk are GE measures of risk if and only if the orderings of all the portfolio vectors are the same as the ordering of the market portfolio.

\subsection{Conclusion}

In this chapter we discussed coherent and spectral measures of risk from a general equilibrium (GE) perspective. Coherent measures of risk can be defined by four axioms: monotonicity, subadditivity, positive homogeneity, translation invariance. Adding two more axioms, law invariance and comonotonic additivity leads to spectral measures of risk. We considered the discrete setting and a general domain $V \subseteq \mathbb{R}^{S}$. We proved that it is also true in the discrete setting with unrestricted domain, that is if $V=\mathbb{R}^{S}$ that spectral measures of risk are the only coherent measures of risk satisfying law invariance and comonotonic additivity. However, we have shown examples where on a general domain $V$ this may not hold.

We defined a natural measure of risk coming out of a general equilibrium model. The GE measure of risk of a portfolio is the negative of its equilibrium market price. Checking the properties of GE measures of risk enabled us to assess the above mentioned six axioms. We found that GE measures of risk are coherent measures of risk. This way the four axioms of coherent measures of risk are supported from a general equilibrium perspective. Thus Value at Risk and other non-coherent risk measures cannot be associated with our general equilibrium framework. 
However, GE measures of risk do not satisfy law invariance, but only a generalized version of it, in which the market portfolio is also taken into account. Since spectral measures of risk are law invariant, we can conclude that in general, when calculating the risk of a regulated entity, spectral measures of risk do not take into account its relation to the market portfolio, leading to an underor overestimation of risk. The same idea is shown by our result that spectral measures of risk are GE measures of risk if and only if all the regulated entities are comonotonic with the market portfolio, that is their values go up and down together event by event. Finally, we showed that GE measures of risk are the only measures of risk satisfying the pricing kernel property, which means that any nonnegative pricing kernel can induce them as the negative of the equilibrium market price. 


\section{Chapter 3}

\section{Balancedness Conditions for Exact Games}

In this chapter ${ }^{1}$ we provide two new characterizations of exact games. First, a game is exact if and only if it is exactly balanced; and second, a game is exact if and only if it is totally balanced and overbalanced.

The condition of exact balancedness is identical to the one of balancedness, except that one of the balancing weights may be negative while for overbalancedness one of the balancing weights is required to be non-positive and no weight is put on the grand coalition. Exact balancedness and overbalancedness are both easy to formulate conditions with a natural game-theoretic interpretation and are shown to be useful in applications.

Using exact balancedness we show that exact games are convex for the grand coalition and we provide an alternative proof that the classes of convex and totally exact games coincide. We provide an example of a game that is totally balanced and convex for the grand coalition, but not exact. Finally we relate classes of balanced, totally balanced, convex for the grand coalition, exact, totally exact, and convex games to one another.

\subsection{Introduction}

It is well-known that the core in a transferable utility game is non-empty if and only if the game is balanced (Bondareva, 1963; Shapley, 1967). The core of every subgame of a transferable utility game is non-empty if and only if the game is totally balanced. Totally balanced games arise from a wide range of applications. They coincide with market games (Shapley and Shubik, 1969); also with a special case of market games with a continuum of indivisible commodities (Legut,

\footnotetext{
${ }^{1}$ This chapter is based on Csóka, Herings, and Kóczy (2007a).
} 
1990); they are equivalent to a class of maximum flow problems (Kalai and Zemel, 1982a); and also to permutation games of less than four players (Tijs, Parthasarathy, Potters, and Prassad, 1984). Moreover, totally balanced games are generated by linear production games (Owen, 1975), generalized network problems (Kalai and Zemel, 1982b), and controlled mathematical programming problems (Dubey and Shapley, 1984).

In this chapter we provide a set of linear programming problems to study a subclass of totally balanced games, exact games (Schmeidler, 1972). By the linear programming problems one can easily check whether a game is exact or not. Using the dual of the problems we develop two new characterizations of exact games complementing those by Schmeidler (1972) and Azrieli and Lehrer (2005).

The first characterization of the class of exact games is the condition of exact balancedness. One interpretation of balancedness is that the players can distribute one unit of working time to any coalition and in doing so cannot generate more value than the grand coalition. The condition of exact balancedness is identical to the one of balancedness, except that players in one coalition are allowed to work overtime in any combination of the coalitions, but their best choice is to stick to their original coalition. We show that exact balancedness implies total balancedness.

The second characterization spells out what more than total balancedness is needed to obtain exactness. It says that a game is exact if an only if it is totally balanced and overbalanced. In case of overbalancedness, no weight is put on the grand coalition and one coalition works a nonnegative amount of overtime. From this characterization it follows immediately that an exact game is totally balanced.

The simplicity of our balancedness conditions is helpful in applications. In Chapter 4 we will use total balancedness and overbalancedness to show that risk allocation games with no aggregate uncertainty are exact. Biswas, Parthasarathy, Potters, and Voorneveld (1999) show that totally exact games are convex (and that convex games are totally exact). Using exact balancedness we provide an alternative proof of this result.

We also study games that are convex for the grand coalition, where convexity is only required for coalitions whose union is the set of all players. We show that exact balancedness implies that a game is convex for the grand coalition, which leads to the result that exact games in case of less than four players are convex. Using the intuition behind exact balancedness, we provide an example of a game which is both totally balanced and convex for the grand coalition, but which 
is not exact.

The structure of the chapter is as follows. We start with the notation and the necessary definitions. In Section 3.3 we study the balancedness conditions for exact games. In Section 3.4 we demonstrate the usefulness of our characterizations of exact games. We conclude the chapter with a summary of how the classes of balanced, totally balanced, convex for the grand coalition, exact, totally exact, and convex games are related to one another.

\subsection{Notation and Definitions}

Let $N=\{1, \ldots, n\}$ denote a finite set of players, $\mathcal{N}$ is the collection of non-empty subsets of $N$, and $\mathcal{D}$ is the collection of non-empty subsets of a coalition $D \in \mathcal{N}$. A value function $v: 2^{N} \rightarrow \mathbb{R}$ satisfying that $v(\emptyset)=0$ gives rise to a cooperative game with transferable utility (game, for short) $(N, v)$. Let $\Gamma$ denote the set of games with $n$ players. An allocation is a vector $x \in \mathbb{R}^{n}$, where $x_{i}$ is the payoff of player $i \in N$. For a coalition $C \in \mathcal{N}$, let $x(C)=\sum_{i \in C} x_{i}$. An allocation $x \in \mathbb{R}^{n}$ is called efficient if $x(N)=v(N)$, individually rational if $x_{i} \geq v(\{i\})$ for all $i \in N$, and coalitionally rational if $x(C) \geq v(C)$ for all $C \in \mathcal{N}$. The core is the set of efficient and coalitionally rational allocations.

For each $C \in \mathcal{N}, a(C) \in \mathbb{R}^{n}$ is the membership vector in $C$, where $a_{i}(C)=1$ if $i \in C$ and $a_{i}(C)=0$ otherwise.

Definition 3.1. A balanced vector of weights is $\left(\lambda_{C}\right)_{C \in \mathcal{N}}$ such that $\lambda_{C} \in \mathbb{R}_{+}$and $\sum_{C \in \mathcal{N}} \lambda_{C} a(C)=a(N)$. A game $(N, v)$ is balanced if $\sum_{C \in \mathcal{N}} \lambda_{C} v(C) \leq v(N)$ for all balanced vectors of weights $\left(\lambda_{C}\right)_{C \in \mathcal{N}}$.

Let $\Gamma_{\mathrm{b}}$ denote the class of balanced games with $n$ players. A well-known interpretation of balancedness is that if the players distribute one unit of working time to any coalition and each coalition is active during $\lambda_{C}$ time units, then the players cannot generate more value than $v(N)$, the value of the grand coalition. Balancedness is a necessary and sufficient condition for the core in a transferable utility game to be non-empty (Bondareva, 1963; Shapley, 1967).

For a game $(N, v)$ and a coalition $D \in \mathcal{N}$ the subgame $\left(D, v^{D}\right)$ is obtained by restricting $v$ to subsets of $D$. 
Definition 3.2. A game $(N, v)$ is totally balanced if for every $D \in \mathcal{N}$ its subgame $\left(D, v^{D}\right)$ is balanced, that is, if for all $D \in \mathcal{N}$ and for all vectors $\left(\lambda_{C}\right)_{C \in \mathcal{D}}$ such that $\lambda_{C} \in \mathbb{R}_{+}$and $\sum_{C \in \mathcal{D}} \lambda_{C} a(C)=a(D)$, we have $\sum_{C \in \mathcal{D}} \lambda_{C} v(C) \leq v(D)$.

In a totally balanced game every subgame has a non-empty core. Let $\Gamma_{\mathrm{tb}}$ denote the class of totally balanced games with $n$ players.

Schmeidler (1972) introduces exact games.

Definition 3.3. A game $(N, v)$ is exact if for each $C \in 2^{N}$ there exists a core allocation $x$ such that $x(C)=v(C)$.

Let $\Gamma_{\mathrm{e}}$ denote the class of exact games with $n$ players.

Definition 3.4. A game $(N, v)$ is convex if for all $S, T \in 2^{N}$ we have $v(S)+v(T) \leq v(S \cup T)+$ $v(S \cap T)$.

Let $\Gamma_{\mathrm{c}}$ denote the class of convex games with $n$ players. Convex games are exact (Schmeidler, 1972). We now introduce the new notion of convexity for the grand coalition.

Definition 3.5. A game $(N, v)$ is convex for the grand coalition if for all $S, T \in 2^{N}$ such that $S \cup T=N$ we have $v(S)+v(T) \leq v(N)+v(S \cap T)$.

Let $\Gamma_{\text {cg }}$ denote the set of games which are convex for the grand coalition.

Following Biswas, Parthasarathy, Potters, and Voorneveld (1999), we define totally exact games analogously to totally balanced games.

Definition 3.6. A game $(N, v)$ is totally exact if for every $D \in \mathcal{N}$ its subgame $\left(D, v^{D}\right)$ is exact. Let $\Gamma_{\text {te }}$ denote the class of totally exact games with $n$ players.

\subsection{Exact Games and Balancedness}

Consider a game $(N, v) \in \Gamma$. For each coalition $D \in \mathcal{N}$ we develop a linear programming problem related to the game $(N, v)$. The linear program is such that whenever $(N, v)$ is exact, any optimal solution $x^{*}$ is a core allocation satisfying $x^{*}(D)=v(D)$. We denote the linear program by $\mathrm{P}_{v, D}$ and its dual by $\mathrm{P}_{v, D}^{*}$. 


$$
\begin{aligned}
& \min a(N) x \\
& \text { s. t. } \\
& \max \sum_{C \in \mathcal{N}} \lambda_{C} v(C) \\
& \text { s. t. } \\
& \left(\mathrm{P}_{v, D}\right) \quad a(C) x \geq v(C), C \in \mathcal{N} \backslash\{D\} \quad\left(\mathrm{P}_{v, D}^{*}\right) \\
& a(D) x=v(D) \\
& x \in \mathbb{R}^{n} \\
& \sum_{C \in \mathcal{N}} \lambda_{C} a(C)=a(N) \\
& \lambda_{C} \in \mathbb{R}_{+}, C \in \mathcal{N} \backslash\{D\} \\
& \lambda_{D} \in \mathbb{R} \text {. }
\end{aligned}
$$

Let us discuss the primal. The first part of the feasibility constraints requires that $x$ is coalitionally rational for all coalitions but coalition $D$. The second part of the feasibility constraints requires that $x(D)=v(D)$, that is, $x$ should be efficient in the subgame $\left(D, v^{D}\right)$, so a feasible solution yields a core element in the subgame $\left(D, v^{D}\right)$.

If $\left(D, v^{D}\right)$ is a balanced game, then the set of feasible solutions is non-empty. In this case, the subgame $\left(D, v^{D}\right)$ has a core element and the other elements of a feasible solution can be chosen sufficiently large. In this case the set of optimal solutions of $\left(\mathrm{P}_{v, D}\right)$ is also non-empty since the set of feasible solutions is bounded from below. If $\left(D, v^{D}\right)$ is not balanced, then the set of feasible solutions of $\left(\mathrm{P}_{v, D}\right)$ is empty, since then $\left(D, v^{D}\right)$ has no core allocations. If $x^{*}$ is an optimal solution of the primal, then $o_{v, D}=a(N) x^{*}$ is the value of the optimal solution, which by the duality theorem of linear programming is the same as the optimal objective value of the dual. If $\left(\mathrm{P}_{v, D}\right)$ has no optimal solutions, then we define $o_{v, D}=\infty$. The linear programming problems are related to exactness as follows.

Proposition 3.7. A game $(N, v) \in \Gamma$ is exact if and only if for every $D \in \mathcal{N}$ we have that $o_{v, D}=v(N)$.

\section{Proof.}

$(\Rightarrow)$ Take any exact game $(N, v) \in \Gamma_{\mathrm{e}}$ and a coalition $D \in \mathcal{N}$. Any feasible solution to $\left(\mathrm{P}_{v, D}\right)$ has an objective value greater than or equal to $v(N)$. As $(N, v)$ is exact there exists a core element $x^{*}$ such that $x^{*}(D)=v(D)$ and $x^{*}(N)=v(N)$. The vector $x^{*}$ satisfies the feasibility constraints of $\left(\mathrm{P}_{v, D}\right)$ and has objective value $a(N) x^{*}=v(N)$.

$(\Leftarrow)$ Assume that for each $D \in \mathcal{N}$ the value of the optimal solution is $v(N)$. Let $x^{*}$ be an optimal solution of $\left(\mathrm{P}_{v, D}\right)$, so $a(N) x^{*}=v(N)$, that is $x^{*}$ is efficient. The efficiency and the feasibility constraints imply that $x^{*}$ is a core allocation of $(N, v)$ with $x(D)=v(D)$. Thus $(N, v)$ is exact. 
Let us continue by analyzing the dual, $\left(\mathrm{P}_{v, D}^{*}\right)$. It holds that $o_{v, D}=v(N)$ if and only if for all feasible solutions of $\left(\mathrm{P}_{v, D}^{*}\right)$ the value of the objective function is not larger than $v(N)$. Using this observation, Proposition 3.7, and the linear programming problems we have the following corollary.

Corollary 3.8. A game $(N, v) \in \Gamma$ is exact if and only if for every $D \in \mathcal{N}$ and for each vector $\left(\lambda_{C}\right)_{C \in \mathcal{N}}$ such that for $C \in \mathcal{N} \backslash\{D\}, \lambda_{C} \in \mathbb{R}_{+}, \lambda_{D} \in \mathbb{R}$, and $\sum_{C \in \mathcal{N}} \lambda_{C} a(C)=a(N)$ we have $\sum_{C \in \mathcal{N}} \lambda_{C} v(C) \leq v(N)$.

In Theorem 3.11 below, we rewrite the conditions in Corollary 3.8 using exact balancedness, defined as follows.

Definition 3.9. An exactly balanced vector of weights is a vector $\left(\lambda_{C}\right)_{C \in \mathcal{N}}$ such that for some $D \in \mathcal{N}, \lambda_{D} \in \mathbb{R}$, for $C \neq D, \lambda_{C} \in \mathbb{R}_{+}$, and $\sum_{C \in \mathcal{N}} \lambda_{C} a(C)=a(N)$. A game $(N, v)$ is exactly balanced if $\sum_{C \in \mathcal{N}} \lambda_{C} v(C) \leq v(N)$ for all exactly balanced vectors of weights.

Notice that the only difference to the condition of balancedness is that one weight, $\lambda_{D}$, can be arbitrary (negative, zero, or positive). If $\lambda_{D}<0$, then we can interpret $\left|\lambda_{D}\right|$ as the amount of time members of coalition $D$ work in overtime. As an illustration, consider the following example.

Example 3.10. Take a game $(N, v)$ with player set $N=\{1,2,3,4\}$. By defining $\lambda_{\{1\}}=$ $1, \lambda_{\{2\}}=1, \lambda_{\{1,3\}}=1, \lambda_{\{2,4\}}=1, \lambda_{\{1,2\}}=-1$ and by setting all other balancing weights to zero, we obtain an exactly balanced vector of weights, since

$$
a(\{1\})+a(\{2\})+a(\{1,3\})+a(\{2,4\})-a(\{1,2\})=a(\{1,2,3,4\})
$$

For exact balancedness

$$
v(\{1\})+v(\{2\})+v(\{1,3\})+v(\{2,4\})-v(\{1,2\}) \leq v(\{1,2,3,4\})
$$

should hold, which can be written alternatively as

$$
v(\{1\})+v(\{2\})+v(\{1,3\})+v(\{2,4\}) \leq v(\{1,2,3,4\})+v(\{1,2\}) .
$$

Equation (3.1) can be interpreted as follows. Players in the coalition with the negative weight, here coalition $\{1,2\}$, work overtime, each member of $\{1,2\}$ allocating a total amount of $-\lambda_{\{1,2\}}$ extra time units among the coalitions $\{1\},\{2\},\{1,3\}$, and $\{2,4\}$, so player 1 spends $-\lambda_{\{1,2\}}$ 
extra time units in coalitions $\{1\}$ and $\{1,3\}$, and player 2 in coalitions $\{2\}$ and $\{2,4\}$. Equation (3.3) says that the total value generated in doing so cannot exceed the value of working one unit of time in the grand coalition and $-\lambda_{\{1,2\}}$ time units of overtime in coalition $\{1,2\}$. Equivalently, Equation (3.2) says that the total value generated in doing so with the opportunity cost of this extra effort $-\lambda_{\{1,2\}} v_{\{1,2\}}$ subtracted, the players cannot generate more value than the grand coalition.

Exact balancedness requires that the value of the coalition having the option to work overtime should be sufficiently large. Since in an exactly balanced vector of weights the weight of any coalition can be negative, exact balancedness implies that the value of each coalition sufficiently large for there to be core elements in which the coalition gets its stand alone value.

Exact balancedness is a necessary and sufficient condition for a game to be exact.

Theorem 3.11. A game $(N, v) \in \Gamma$ is exact if and only if it is exactly balanced.

Proof. This follows immediately from Corollary 3.8 .

Exact games can also be characterized by total balancedness and overbalancedness, to be defined next.

Definition 3.12. An overbalanced vector of weights is a vector $\left(\mu_{C}\right)_{C \in \mathcal{N} \backslash\{N\}}$ such that $\mu_{C} \in \mathbb{R}_{+}$ and $\sum_{C \in \mathcal{N} \backslash\{D, N\}} \mu_{C} a(C)=a(N)+\mu_{D} a(D)$ for some $D \in \mathcal{N}$. A game $(N, v)$ is overbalanced if $\sum_{C \in \mathcal{N} \backslash\{D, N\}} \mu_{C} v(C) \leq v(N)+\mu_{D} v(D)$ for all overbalanced vectors of weights.

The difference between balancedness and overbalancedness is that in the case of overbalancedness, no weight is put on the grand coalition and the members of one coalition are forced to work a non-negative amount of overtime. As in Equation (3.3), the overbalancedness condition requires that working one unit of time for the grand coalition and a non-negative amount of overtime in coalition $D$ generates at least the same value as any allocation of the same amount of time to work in the other coalitions.

Theorem 3.13. A game $(N, v) \in \Gamma$ is exact if and only if it is totally balanced and overbalanced.

Proof. We show that a game $(N, v) \in \Gamma$ is exactly balanced if and only if it is totally balanced and overbalanced. 
$(\Rightarrow)$ Total balancedness of $(N, v)$ follows almost immediately by the definition of exactness, or one obtains it using exact balancedness as follows. For $D \in \mathcal{N} \backslash\{N\}$ balancedness of $\left(D, v^{D}\right)$ is obtained by setting the exactly balanced weights such that $\lambda_{N}=1$ and $\lambda_{D}=-1$. Balancedness of $(N, v)$ is obtained by considering exactly balanced vectors of weights where all the weights are non-negative.

Using exactly balanced vectors of weights with $\lambda_{N}=0$ and $\lambda_{D} \leq 0$ implies overbalancedness of $(N, v)$.

$(\Leftarrow)$ Take any exactly balanced vector of weights $\left(\lambda_{C}\right)_{C \in \mathcal{N}}$ with $\lambda_{D} \in \mathbb{R}, \lambda_{C} \in \mathbb{R}_{+}$for $C \in$ $\mathcal{N} \backslash\{D\}$, and

$$
\sum_{C \in \mathcal{N}} \lambda_{C} a(C)=a(N)
$$

It follows immediately that $\lambda_{N} \leq 1$. We discuss two cases depending on the value of $\lambda_{N}$.

1. $\lambda_{N}<1$. In this case (3.4) can be rearranged as

$$
\sum_{C \in \mathcal{N} \backslash\{N\}} \frac{\lambda_{C}}{1-\lambda_{N}} a(C)=a(N) .
$$

If $\lambda_{D} \leq 0$, then exact balancedness is implied by overbalancedness of $(N, v)$, otherwise by balancedness of $(N, v)$.

2. $\lambda_{N}=1$. If $\lambda_{D} \geq 0$, then exact balancedness is trivially satisfied since now $\lambda_{N}=1$ and (3.4) implies $\left(\lambda_{C}\right)_{C \in \mathcal{N} \backslash\{N\}}=0$. If $\lambda_{D}<0$, then (3.4) can be rearranged as

$$
\sum_{C \in \mathcal{N} \backslash\{D, N\}} \frac{\lambda_{C}}{-\lambda_{D}} a(C)=a(D),
$$

and exact balancedness follows from total balancedness of $(N, v)$.

Theorem 3.13 characterizes exact games as being the class of totally balanced and overbalanced games. It follows immediately that an exact game is totally balanced, which was also shown by Schmeidler (1972). Further, the result shows that exact games are not only totally balanced, but also satisfy the extra condition of overbalancedness, where no weight is put on the grand 
coalition and one weight should be non-positive when checking the "normal" balancedness of the game.

In Derks and Reijnierse (1998), Theorem 7, the characterization of exact games by Schmeidler (1972), is formulated as follows.

Theorem 3.14. (Schmeidler, 1972) A game $(N, v) \in \Gamma$ is exact if and only if for every $D \in \mathcal{N} \backslash$ $\{N\}$, for each vector $\left(\gamma_{C}\right)_{C \in \mathcal{N}}$ such that $\gamma_{C} \in \mathbb{R}_{+}$and $\sum_{C \in \mathcal{N} \backslash\{N\}} \gamma_{C} a(C)=a(D)+\gamma_{N} a(N)$ we have $\sum_{C \in \mathcal{N} \backslash\{N\}} \gamma_{C} v(C) \leq v(D)+\gamma_{N} v(N)$.

To clarify the differences, we provide a direct proof of the equivalence of Schmeidler's characterization of exactness and the characterization in Theorem 3.13.

Proposition 3.15. A game $(N, v) \in \Gamma$ satisfies the conditions in Theorem 3.14 if and only if it is totally balanced and overbalanced.

\section{Proof.}

$(\Rightarrow)$ That $\left(D, v^{D}\right)$ is balanced for $D \in \mathcal{N} \backslash\{N\}$ follows by setting $\gamma_{N}=0$. Balancedness of $(N, v)$ follows from weights such that $\gamma_{N}=1$ and $\gamma_{D} \geq 1$. Overbalanced weights satisfy the equality

$$
\sum_{C \in \mathcal{N} \backslash\{D, N\}} \mu_{C} a(C)=a(N)+\mu_{D} a(D) .
$$

The case where $\mu_{D}=0$ follows from Schmeidler's characterization with $\gamma_{N}=1$ and $\gamma_{D}=1$. In case $\mu_{D}>0,(3.7)$ is equivalent to

$$
\sum_{C \in \mathcal{N} \backslash\{D, N\}} \frac{\mu_{C}}{\mu_{D}} a(C)=\frac{1}{\mu_{D}} a(N)+a(D) .
$$

It is now easily seen that this case is implied by Schmeidler's characterization when we choose $\gamma_{D}=0, \gamma_{N}=1 / \mu_{D}$, and $\gamma_{C}=\mu_{C} / \mu_{D}$ for $C \in \mathcal{N} \backslash\{D, N\}$.

$(\Leftarrow)$ The balancing weights of Schmeidler satisfy

$$
\sum_{C \in \mathcal{N} \backslash\{N\}} \gamma_{C} a(C)=a(D)+\gamma_{N} a(N) .
$$

In this case Schmeidler's condition is $\sum_{C \in \mathcal{N} \backslash\{N\}} \gamma_{C} v(C) \leq v(D)+\gamma_{N} v(N)$. If $\gamma_{N}=0$, then balancedness of $\left(D, v^{D}\right)$ gives rise to Schmeidler's condition. If $\gamma_{N}>0$ and $\gamma_{D} \geq 1$, then (3.8) 
is equivalent to

$$
\sum_{C \in \mathcal{N} \backslash\{D, N\}} \frac{\gamma_{C}}{\gamma_{N}} a(C)+\frac{\gamma_{D}-1}{\gamma_{N}} a(D)=a(N),
$$

and Schmeidler's condition follows from balancedness. If $\gamma_{N}>0$ and $\gamma_{D}<1$, then (3.8) is equivalent to

$$
\sum_{C \in \mathcal{N} \backslash\{D, N\}} \frac{\gamma_{C}}{\gamma_{N}} a(C)=a(N)+\frac{1-\gamma_{D}}{\gamma_{N}} a(D),
$$

so Schmeidler's condition follows from overbalancedness.

For a coalition $C \in \mathcal{N}$ let $|C|$ denote the number of players involved in the coalition. Azrieli and Lehrer (2005) give the following necessary and sufficient conditions for exactness.

Theorem 3.16. (Azrieli and Lehrer, 2005, Proposition 2) A game $(N, v) \in \Gamma$ is exact if and only iffor every $D \in \mathcal{N}$, for each vector $\left(\alpha_{C}\right)_{C \in \mathcal{N}}$ such that $\alpha_{C} \in \mathbb{R}_{+}, \sum_{C \in \mathcal{N}} \alpha_{C}=1, \beta \in[0,1]$ and $\sum_{C \in \mathcal{N}} \alpha_{C} \frac{a(C)}{|C|}=\beta \frac{a(D)}{|D|}+(1-\beta) \frac{a(N)}{|N|}$ we have $\sum_{C \in \mathcal{N}} \alpha_{C} \frac{v(C)}{|C|} \leq \beta \frac{v(D)}{|D|}+(1-\beta) \frac{v(N)}{|N|}$.

Notice that in Theorem 3.16 if $D=N$, we have the usual balancedness condition expressed in terms of average worth, that is when distributing one unit of working time to the coalitions, players cannot generate more value per person than in the grand coalition.

In a similar way as in the proof of Proposition 3.15, one can show that the characterization by Azrieli and Lehrer (2005) is equivalent to the characterization by total balancedness and overbalancedness.

Of all the characterizations presented, exact balancedness is closest to balancedness. In fact, the only difference is that in exact balancedness one of the balancing weights can be chosen to be negative. The characterization of exact games as the ones that satisfy total balancedness and overbalancedness spells out what more than total balancedness is needed to obtain exactness.

\subsection{Applications of Exact Balancedness}

In Chapter 4 we will use the characterization of exact games as those that are totally balanced and overbalanced to show that risk allocation games with no aggregate uncertainty are exact. In this section we show how the condition of exact balancedness (Definition 3.9) can be used 
to show the relationships between the various classes of games defined in Section 3.2. First we demonstrate that exact games are convex for the grand coalition (Definition 3.5) and that the class of totally exact games (Definition 3.6) coincides with the class of convex games (Definition 3.4). Then we discuss games with less than four players, where exact games turn out to be convex. Finally, we present an example of a game with four players that is totally balanced, convex for the grand coalition, but not exact.

Proposition 3.17. If the game $(N, v)$ is exact, then it is convex for the grand coalition, $\Gamma_{\mathrm{e}} \subseteq \Gamma_{\mathrm{cg}}$.

Proof. Take two coalitions $S, T \in 2^{N}$ such that $S \cup T=N$. Let $D=S \cap T$. Since $(N, v)$ is exact, by Theorem 3.11 it is exactly balanced. We define an exactly balanced vector of weights by setting $\lambda_{S}=1, \lambda_{T}=1$, and $\lambda_{D}=-1$. All other balancing weights are set to zero. Notice that this constitutes an exactly balanced vector of weights, since $a(S)+a(T)-a(D)=a(N)$. By exact balancedness we have that $v(S)+v(T)-v(D) \leq v(N)$, that is $v(S)+v(T) \leq v(N)+v(S \cap T)$.

Theorem 3.18. A game is totally exact if and only if it is convex, $\Gamma_{\text {te }}=\Gamma_{c}$.

\section{Proof.}

$(\Rightarrow)$ Since by Proposition 3.17 exact games are convex for the grand coalition, totally exact games are convex for all coalitions, that is they are convex.

$(\Leftarrow)$ It is known that convex games are exact (Schmeidler, 1972). Since a subgame of a convex game is also convex, we have total exactness.

The following result claims that a game with less than four players that is totally balanced and convex for the grand coalition has to be convex.

Proposition 3.19. Assume that there are less than four players. If the game $(N, v)$ is totally balanced and convex for the grand coalition, then it is convex.

Proof. For one or two player games the claim requires no proof. Consider the case with three players. For coalitions $S, T \in 2^{N}$ such that $S \cup T=N$ convexity follows from the convexity for the grand coalition. When $S \cap T=\emptyset, S \cup T=C$, where $C$ is a two-player coalition, convexity follows from total balancedness. When $S \subseteq T$, where $T$ is a two-player coalition, convexity 
follows since $S \cap T=S$ and $S \cup T=T$.

Exact games are totally balanced (Theorem 3.13) and convex for the grand coalition (Proposition 3.17): $\Gamma_{\mathrm{e}} \subseteq \Gamma_{\mathrm{tb}} \cap \Gamma_{\mathrm{cg}}$. Since a convex game is exact, using Proposition 3.19 we have the following corollary.

Corollary 3.20. Assume that there are less than four players. Then a game is exact if and only if it is convex.

There exist games that are convex for the grand coalition, but that are not totally balanced, and games that are totally balanced, but not convex for the grand coalition, so $\Gamma_{\mathrm{tb}} \cap \Gamma_{\mathrm{cg}} \subsetneq \Gamma_{\mathrm{cg}}$ and $\Gamma_{\mathrm{tb}} \cap \Gamma_{\mathrm{cg}} \subsetneq \Gamma_{\mathrm{tb}}$. Theorem 3.13 and Proposition 3.17 imply that $\Gamma_{\mathrm{e}} \subseteq \Gamma_{\mathrm{tb}} \cap \Gamma_{\mathrm{cg}}$. We consider next the question whether there are games that are totally balanced and convex for the grand coalition, but not exact. Using the intuition behind exact balancedness we provide an example of such a game, thus we have that $\Gamma_{\mathrm{e}} \subsetneq \Gamma_{\mathrm{tb}} \cap \Gamma_{\mathrm{cg}}$.

Example 3.21. We present a game that is totally balanced and convex for the grand coalition, but not exact. By Proposition 3.19 we need at least four players. Consider the following game with four players. Let

$$
\begin{aligned}
& v(\{1\})=v(\{2\})=v(\{3\})=v(\{4\})=0, \\
& v(\{1,2\})=v(\{1,3\})=v(\{1,4\})=1, \\
& v(\{2,3\})=v(\{2,4\})=v(\{3,4\})=0, \\
& v(\{1,2,3\})=v(\{1,2,4\})=v(\{1,3,4\})=1, \\
& v(\{2,3,4\})=0, \\
& v(\{1,2,3,4\})=2 .
\end{aligned}
$$

The game $(N, v)$ is totally balanced, since every subgame $\left(C, v^{C}\right)$ with $C$ containing player 1 has a core element where player 1 receives $v(C)$ and the other players nothing, and the zero vector is a core element of all other subgames. The game $(N, v)$ is convex for the grand coalition as $v(N)=$ 2 weakly exceeds the sum of the value of any other two coalitions. We show that the game $(N, v)$ is not exactly balanced. Let $D=\{1\}$ and consider the weights $\lambda_{\{1,2\}}=\lambda_{\{1,3\}}=\lambda_{\{1,4\}}=$ $1, \lambda_{\{1\}}=-2$, and set all other weights equal to zero. These weights constitute an exactly 
balanced vector of weights, since $a(\{1,2\})+a(\{1,3\})+a(\{1,4\})-2 a(\{1\})=a(\{1,2,3,4\})$. However, $v(\{1,2\})+v(\{1,3\})+v(\{1,4\})-2 v(\{1\})=3>2=v(\{1,2,3,4\})$, thus $v$ is not exact.

\subsection{Conclusion}

In this chapter we have provided two new characterizations of exact games using linear programming formulations. The first characterization of exactness is by exact balancedness. The condition of exact balancedness is identical to the one of balancedness, except that exactly one of the weights is allowed to be negative. Using the usual interpretation of balancedness, this is equivalent to saying that players are allowed to work overtime in a particular coalition.

The second one says that a game is exact if and only if it is totally balanced and overbalanced. The condition of overbalancedness requires one coalition to work a non-negative amount of overtime.

By applying exact balancedness we have shown that the class of totally exact games $\left(\Gamma_{\text {te }}\right)$ coincides with the class of convex games $\left(\Gamma_{\mathrm{c}}\right)$. We have also proven that with less than four players the class of exact games coincides with the class of convex games.

Our characterization of exact games by total balancedness and overbalancedness implies that an exact game is totally balanced $\left(\Gamma_{\mathrm{tb}}\right)$, and we have shown that exact balancedness implies that an exact game is convex for the grand coalition $\left(\Gamma_{\mathrm{cg}}\right)$. Finally, we have provided an example of a game that is totally balanced and convex for the grand coalition, but not exact. The relationships between the various classes of games are summarized in Figure 3.1. 


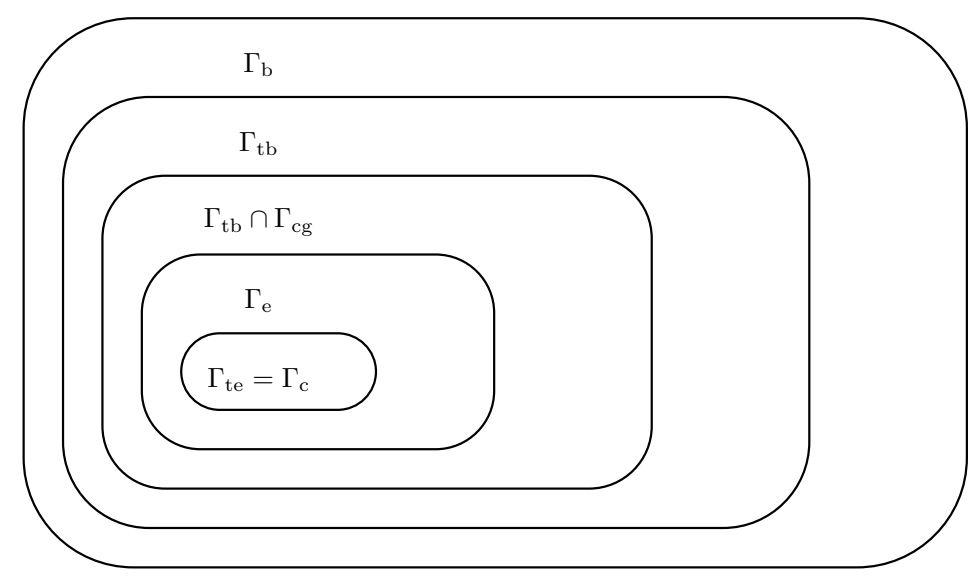

Figure 3.1: Subsets of balanced games. 


\section{Chapter 4}

\section{Stable Allocations of Risk}

The measurement and the allocation of risk are fundamental problems of portfolio management. Coherent measures of risk provide an axiomatic approach to the former problem. In an environment given by a coherent measure of risk and the various portfolios' realization vectors, risk allocation games aim at solving the second problem: How to distribute the diversification benefits of the various portfolios? Understanding these cooperative games helps us to find stable, efficient, and fair allocations of risk.

In this chapter ${ }^{1}$ we show that the class of risk allocation and totally balanced games coincide, hence a stable allocation of risk is always possible. When the aggregate portfolio is riskless, the class of risk allocation games coincides with the class of exact games. As in exact games any subcoalition may be subject to marginalization even in core allocations, our result further emphasizes the responsibility involved in allocating risk.

\subsection{Introduction}

The value of an investment portfolio is subject to all kinds of uncertain events. Firms, banks, or insurance companies (to which we refer by the term portfolios) face risk and regulators may require them to hold cash reserves as a cushion against default - this rather unfavorable state of nature - with the precise amounts determined by a measure of risk. A measure of risk thereby specifies the minimal amount of cash the regulated agent has to add to his portfolio for his risk to be acceptable to the regulator.

The literature knows of numerous possible ways to measure risk; lately interest shifted to coher-

\footnotetext{
${ }^{1}$ This chapter is based on Csóka, Herings, and Kóczy (2007c).
} 
ent measures of risk (Artzner, Delbaen, Eber, and Heath, 1999) defined by four axioms: monotonicity, subadditivity, positive homogeneity, and translation invariance. These axioms have been shown to be compatible with a natural general equilibrium approach to measure risk in Chapter 2.

Of these axioms, subadditivity expresses that the risk of an aggregate portfolio should not exceed the total risk of the individual subportfolios. In particular, the risk of a firm is less than the sum of the risks of the constituents of the firm. Risk allocation then addresses the distribution of the diversification benefits; risk allocation games (Denault, 2001) are transferable utility games defined to this purpose.

A risk allocation game assigns to each coalition of portfolios the risk involved in the aggregate portfolio of the coalition. An allocation shows how to share the risk of the aggregate portfolio of the grand coalition among the individual portfolios, which has of course consequences on the cash reserves to be held. The allocation makes clear what part of the risk of a firm should be attributed to each of its constituents. A natural question that arises is whether there are stable allocations of risk, allocations of risk that no coalition can object to, that is whether the core of the risk allocation game is non-empty.

We separate the risk environment specifying the individual portfolios' realization vectors of discrete random variables and a coherent measure of risk, a real valued function on the realization vectors, from the derived cooperative game that we call risk allocation game.

A totally balanced game is a cooperative game having a non-empty core in all of its subgames. Totally balanced games arise from a wide range of applications. They coincide with market games (Shapley and Shubik, 1969); also with a special case of market games with a continuum of indivisible commodities: cooperation in fair division (Legut, 1990); they are equivalent to a class of maximum flow problems (Kalai and Zemel, 1982a); and also to permutation games of less than four players (Tijs, Parthasarathy, Potters, and Prassad, 1984). Moreover, totally balanced games are generated by linear production games (Owen, 1975), generalized network problems (Kalai and Zemel, 1982b), and controlled mathematical programming problems (Dubey and Shapley, 1984).

We show that the class of risk allocation games coincides with the class of totally balanced games, that is all risk allocation games are totally balanced and all totally balanced games can be generated by a risk allocation game with a properly specified risk environment. This result ensures 
that a regulator can always allocate risk in a stable way. No matter how the risk environment changes, there is always a core element.

We next provide a linear program such that its optimal objective value can be used to determine whether a given cooperative game is a risk allocation game or not. If the game is a risk allocation game, then an optimal solution to the linear program yields a risk environment that generates the game. We then show how to use the linear program to characterize all risk environments that generate a given totally balanced game.

At last, we focus on games where only the distribution of values is uncertain, while the value of the aggregate portfolio is constant over all states of nature. This case is relevant for situations where the risk of the aggregate portfolio is low compared to the risk involved in the individual portfolios. We show that the class of risk allocation games with no aggregate uncertainty coincides with the class of exact games (Schmeidler, 1972).

The fact that each risk allocation game is exact implies that for each coalition there is a core element such that the coalition only gets its stand-alone value. This means that in the case of no aggregate uncertainty, this coalition does not necessarily benefit from the diversification opportunities offered by the aggregate portfolio. As a consequence, the regulator has a high level of discretion in allocating the risk to the individual portfolios.

As evidenced by the previous paragraphs, there are many applications giving rise to the class of totally balanced games; to the best of our knowledge, risk allocation with no aggregate uncertainty is the first application that leads to the full class of exact games. However, Calleja, Borm, and Hendrickx (2005) show that the class of multi-issue allocation games coincides with the class of non-negative exact games.

The structure of the chapter is as follows. First we introduce coherent measures of risk, transferable utility games, and risk allocation games. In Section 4.3 we prove that the class of risk allocation games coincides with the class of totally balanced games and investigate our constructive proof by linear programming. In Section 4.4 we show that the class of risk allocation games with no aggregate uncertainty coincides with the class of exact games. In Section 4.5 we conclude. 


\subsection{Preliminaries}

\subsubsection{Coherent Measures of Risk}

Consider the set $\mathbb{R}^{S}$ of realization vectors, where $S$ denotes the number of states of nature. State of nature $s$ occurs with probability $p_{s}>0$ and $\sum_{s=1}^{S} p_{s}=1$. The vector $X \in \mathbb{R}^{S}$ represents a portfolio's possible profit and loss realizations on a common chosen future time horizon, say at $t=1$. The amount $X_{s}$ is the portfolio's payoff in state of nature $s$. Negative values of $X_{s}$ correspond to losses. The inequality $Y \geq X$ means that $Y_{s} \geq X_{s}$ for all $s=1, \ldots, S$.

A measure of risk is a function $\rho: \mathbb{R}^{S} \rightarrow \mathbb{R}$ measuring the risk of a portfolio from the perspective of the present $(t=0)$. It is the minimal amount of cash the regulated agent has to add to his portfolio, and to invest in a reference instrument today, such that it ensures that the risk involved in the portfolio is acceptable to the regulator. We assume that the reference instrument has payoff 1 in each state of nature at $t=1$, thus its realization vector is $1^{S}=(1, \ldots, 1)^{\top}$. The reference instrument is riskless in the "classical sense," having no uncertainty in its payoffs. It is most natural to think of it as a zero coupon bond. The price of the reference instrument is denoted by $\delta \in \mathbb{R}_{+}$, where $\mathbb{R}_{+}=[0, \infty)$. We adjust the definition of coherent measures of risk to the discrete case with realization vectors as follows.

Definition 4.1. A function $\rho: \mathbb{R}^{S} \rightarrow \mathbb{R}$ is called a coherent measure of risk (Artzner et al., 1999) if it satisfies the following axioms.

1. Monotonicity: for all $X, Y \in \mathbb{R}^{S}$ such that $Y \geq X$, we have $\rho(Y) \leq \rho(X)$.

2. Subadditivity: for all $X, Y \in \mathbb{R}^{S}$, we have $\rho(X+Y) \leq \rho(X)+\rho(Y)$.

3. Positive homogeneity: for all $X \in \mathbb{R}^{S}$ and $h \in \mathbb{R}_{+}$, we have $\rho(h X)=h \rho(X)$.

4. Translation invariance: for all $X \in \mathbb{R}^{S}$ and $a \in \mathbb{R}$, we have $\rho\left(X+a 1^{S}\right)=\rho(X)-\delta a$.

Acerbi (2002) treats a subclass of coherent measures of risk: spectral measures of risk. The definition of spectral measures of risk with equiprobable outcomes, that is $p_{1}=\cdots=p_{S}=1 / S$, is as follows.

Let us introduce the ordered statistics $X_{s: S}$ given by the ordered values of the $S$-tuple $X_{1}, \ldots, X_{S}$, that is $\left\{X_{1: S}, \ldots, X_{S: S}\right\}=\left\{X_{1}, \ldots, X_{S}\right\}$ and $X_{1: S} \leq X_{2: S} \leq \cdots \leq X_{S: S}$. 
Definition 4.2. Let the outcomes be equiprobable. Consider a vector $\phi \in \mathbb{R}^{S}$. The measure of risk $M_{\phi}: \mathbb{R}^{S} \rightarrow \mathbb{R}$ defined by

$$
M_{\phi}(X)=-\delta \sum_{s=1}^{S} \phi_{s} X_{s: S}
$$

is a spectral measure of risk if $\phi \in \mathbb{R}^{S}$ satisfies the conditions

1. Nonnegativity: $\phi_{s} \geq 0$ for all $s=1, \ldots, S$,

2. Normalization: $\sum_{s=1}^{S} \phi_{s}=1$,

3. Monotonicity : $\phi_{s}$ is non-increasing, that is $\phi_{s_{1}} \geq \phi_{s_{2}}$ if $s_{1}<s_{2}$ and $s_{1}, s_{2} \in\{1, \ldots, S\}$.

Spectral measures of risk are discounted weighted average losses, with non-increasing weights, with the highest weight on the worst outcome. The weight vector $\phi$ is the so-called risk spectrum, the "attitude" toward risk. An important example of a spectral measure of risk is the $k$-expected shortfall.

Definition 4.3. Let the outcomes be equiprobable and let $k \in\{1, \ldots, S\}$. The $k$-expected shortfall of the realization vector $X$ is defined by

$$
\operatorname{ES}_{k}(X)=-\delta \sum_{s=1}^{k} \frac{1}{k} X_{s: S} .
$$

The $k$-expected shortfall is the discounted average of the worst $k$ outcomes. For a detailed discussion see Acerbi and Tasche (2002).

\subsubsection{Transferable Utility Games}

Let $N=\{1, \ldots, n\}$ denote a finite set of players. A value function $v: 2^{N} \rightarrow \mathbb{R}$ with $v(\{\emptyset\})=0$ gives rise to a cooperative game with transferable utility (game, for short) $(N, v)$. Let $\Gamma$ denote the set of games with $n$ players. An allocation is a vector $x \in \mathbb{R}^{n}$, where $x_{i}$ is the payoff of player $i \in N$. An allocation $x$ yields payoff $x(C)=\sum_{i \in C} x_{i}$ to a coalition $C \in 2^{N}$. An allocation $x \in \mathbb{R}^{n}$ is called efficient, if $x(N)=v(N)$; individually rational, if $x_{i} \geq v(\{i\})$ for all $i \in N$, and coalitionally rational if $x(C) \geq v(C)$ for all $C \in 2^{N}$. The core is the set of efficient and coalitionally rational allocations.

For each $C \in 2^{N}$ let $a(C) \in \mathbb{R}^{n}$ be the membership vector, $a_{i}(C)=1$ for $i \in C$ and $a_{i}(C)=0$ otherwise. 
Definition 4.4. A balanced vector of weights is a vector $\left(\lambda^{C}\right)_{C \in 2^{N}} \in \mathbb{R}_{+}^{2^{N}}$ such that $\sum_{C \in 2^{N}} \lambda^{C} a(C)=a(N)$. A game $(N, v)$ is balanced if $\sum_{C \in 2^{N}} \lambda^{C} v(C) \leq v(N)$ for all balanced vectors of weights.

A well-known interpretation of balancedness is that the players can distribute one unit of working time to any coalition and if each coalition is active during a fraction $\lambda^{C}$ of a unit of time then the players cannot generate more value than $v(N)$, the value of the grand coalition. Balancedness is a necessary and sufficient condition for non-emptiness of the core in a transferable utility game (Bondareva, 1963; Shapley, 1967). See Predtetchinski and Herings (2004) for an extension of the concept of balancedness to be necessary and sufficient for non-emptiness of the core in nontransferable utility games.

For a game $(N, v)$ and a coalition $C \in 2^{N}$, a subgame $\left(C, v^{C}\right)$ is obtained by restricting $v$ to subsets of $C$.

Definition 4.5. A game $(N, v)$ is totally balanced if for every $D \in 2^{N}$ its subgame $\left(D, v^{D}\right)$ is balanced, that is, if for all $D \in 2^{N}$ and for all vectors $\left(\lambda^{C}\right)_{C \in 2^{D}} \in \mathbb{R}_{+}^{2^{D}}$ satisfying $\sum_{C \in 2^{D}} \lambda^{C} a(C)=$ $a(D)$, we have $\sum_{C \in 2^{D}} \lambda^{C} v(C) \leq v(D)$.

In a totally balanced game every subgame has a non-empty core. Let $\Gamma_{\text {tb }}$ denote the family of totally balanced games with $n$ players. An interesting subclass of totally balanced games is the class of exact games (Schmeidler, 1972).

Definition 4.6. A game $(N, v)$ is exact if for each $C \in 2^{N}$ there exists a core allocation $x$ such that $x(C)=v(C)$.

Schmeidler (1972) characterizes exact games as follows (see also Derks and Reijnierse (1998), Theorem 7).

Theorem 4.7. A game $(N, v)$ is exact if and only if for all $D \in 2^{N} \backslash\{N\}$ and for all vectors $\left(\gamma^{C}\right)_{C \in 2^{N}} \in \mathbb{R}_{+}^{2^{N}}$ satisfying $\sum_{C \in 2^{N} \backslash\{N\}} \gamma^{C} a(C)=\gamma^{N} a(N)+a(D)$, we have $\sum_{C \in 2^{N} \backslash\{N\}} \gamma^{C} v(C)$ $\leq \gamma^{N} v(N)+v(D)$.

In Chapter 3 we have introduced the concept of overbalancedness.

Definition 4.8. An overbalanced vector of weights is a vector $\left(\lambda^{C}\right)_{C \in 2^{N}} \in \mathbb{R}_{+}^{2^{N}}$ such that $\sum_{C \in 2^{N} \backslash\{D, N\}} \lambda^{C} a(C)-\lambda^{D} a(D)=a(N)$ for some $D \in 2^{N}$. A game $(N, v)$ is overbalanced if $\sum_{C \in 2^{N} \backslash\{D, N\}} \lambda^{C} v(C)-\lambda^{D} v(D) \leq v(N)$ for all overbalanced vectors of weights. 
A natural interpretation of overbalancedness is that if one coalition is forced to exist for a nonpositive amount of time $\left(-\lambda^{D} \leq 0\right)$, which requires its players to spend $\left(1+\lambda^{D}\right)$ units of time in the other coalitions, the players cannot generate more value than $v(N)$.

Let $\Gamma_{\mathrm{e}}$ denote the family of exact games with $n$ players. In Chapter 3 we gave the following necessary and sufficient condition for a game to be exact as an alternative to the conditions of Theorem 4.7.

Theorem 4.9. The game $(N, v)$ belongs to $\Gamma_{\mathrm{e}}$ if and only if it is totally balanced and overbalanced.

Convex games (Shapley, 1971) are a subset of exact games.

Definition 4.10. A game $(N, v)$ is convex if for all $C, D \in 2^{N}$ we have that $v(C)+v(D) \leq v(C \cup D)+v(C \cap D)$.

Let $\Gamma_{\mathrm{c}}$ denote the family of convex games with $n$ players. We have that $\Gamma \supseteq \Gamma_{\mathrm{tb}} \supseteq \Gamma_{\mathrm{e}} \supseteq \Gamma_{\mathrm{c}}$.

\subsubsection{Risk Allocation Games}

Denault (2001) introduces risk capital allocation problems: Suppose a firm has $n$ constituents and the matrix of their realization vectors is given by $X \in \mathbb{R}^{S \times n} .^{2}$ The question is how the risk of the firm as measured by a coherent measure of risk has to be allocated to its constituents in a stable way, meaning that the risk allocated to a coalition of constituents does not exceed the risk of the coalition's aggregate portfolio.

Let $X_{. i}$ denote the $i$-th column of $X$, the realization vector of portfolio $i$. Let $X_{s}$. denote the row of $X$ corresponding to state of nature $s, X_{s, i}$ its element at row $s$ and column $i$, and $\left(X_{s, i}\right)_{i \in D}$ the row vector corresponding to state of nature $s$ with elements $i \in D$. For a coalition of portfolios $C \in 2^{N}$, let $X(C)=\sum_{i \in C} X_{\cdot i}$ and $X_{s}(C)=\sum_{i \in C} X_{s, i}$.

Denault (2001) assumes that the $n$-th portfolio equals $b \in \mathbb{R}$ units of the reference instrument: $X_{._{n}}=b 1^{S}$. We will consider the slightly more general setting where $X_{._{n}}$ can be any portfolio. Moreover, we make a distinction between the risk environment and the induced game.

\footnotetext{
${ }^{2}$ Denault (2001) uses continuously distributed random variables. We adjust his setting to the more tractable setup with discrete random variables, resulting in realization vectors.
} 
Definition 4.11. A risk environment is a tuple $(N, S, p, X, \rho)$, where $N$ is the set of portfolios, $S$ indicates the number of states of nature, $p=\left(p_{1}, \ldots, p_{S}\right)$ is the vector of realization probabilities of the various states, $X$ is the matrix of realization vectors, and $\rho$ is a coherent measure of risk.

Definition 4.12. Given a risk environment $(N, S, p, X, \rho)$ a risk allocation game is a game $(N, v)$, where the value function $v: 2^{N} \rightarrow \mathbb{R}$ is defined by

$$
v(C)=-\rho(X(C)) \text { for all } C \in 2^{N} .
$$

A risk allocation game with $n$ players is induced by the number of states of nature, their probability of occurrence, $n$ realization vectors and a coherent measure of risk. Let $\Gamma_{\mathrm{r}}$ denote the family of risk allocation games with $n$ players. In such a game, according to Equation (4.3), the larger the risk of any subset of portfolios, the lower its value.

If the rows of a matrix of realization vectors sum up to the same number, then there is no aggregate uncertainty. Formally:

Definition 4.13. A matrix of realization vectors $X \in \mathbb{R}^{S \times n}$ has no aggregate uncertainty if there exists a number $\alpha \in \mathbb{R}$ such that $X(N)=\alpha 1^{S}$.

Let $\Gamma_{\text {rnau }}$ denote the family of risk allocation games with $n$ players with no aggregate uncertainty. Obviously, $\Gamma_{\text {rnau }} \subseteq \Gamma_{\mathrm{r}}$. We first study risk allocation games in general, then with no aggregate uncertainty.

\subsection{Total Balancedness}

\subsubsection{Risk Allocation Games and Totally Balanced Games}

Denault (2001, Theorem 4) shows that the family of risk capital allocation problems is balanced. As a subgame of a risk allocation game is also a risk allocation game, we can adjust his proof to show that risk allocation games are totally balanced.

Proposition 4.14. All games $(N, v) \in \Gamma_{\mathrm{r}}$ are totally balanced, $\Gamma_{\mathrm{r}} \subseteq \Gamma_{\mathrm{tb}}$.

Proof. Consider a risk environment $(N, S, p, X, \rho)$ inducing the game $(N, v)$. We show that for any $D \in 2^{N}$, the subgame $\left(D, v^{D}\right)$ is balanced. Take any $\left(\lambda^{C}\right)_{C \in 2^{D}} \in \mathbb{R}_{+}^{2^{D}}$ such that 
$\sum_{C \in 2^{D}} \lambda^{C} a(C)=a(D)$. Then by Equation (4.3) and the positive homogeneity and subadditivity of $\rho$ we have that

$$
\begin{aligned}
\sum_{C \in 2^{D}} \lambda^{C} v^{D}(C) & =-\sum_{C \in 2^{D}} \rho\left(\lambda^{C} X(C)\right) \leq-\rho\left(\sum_{C \in 2^{D}}\left(\sum_{i \in C} \lambda^{C} X_{\cdot i}\right)\right) \\
& =-\rho\left(\sum_{i \in D}\left(\sum_{C \in 2^{D}, C \ni i} \lambda^{C} X_{\cdot i}\right)\right)=-\rho\left(\sum_{i \in D} X_{\cdot i}\right)=-\rho(X(D)) \\
& =v^{D}(D),
\end{aligned}
$$

where the last line follows from rearranging the summation and using the fact that we have a balanced vector of weights. Thus $\left(D, v^{D}\right)$ is balanced.

Not only is it true that all risk allocation games are totally balanced, but also any totally balanced game can be generated by a risk allocation game. We illustrate Proposition 4.15 and its proof by Example 4.16.

Proposition 4.15. Each game $(N, v) \in \Gamma_{\text {tb }}$ is induced by some risk environment $(N, S, p, X, \rho)$, so $\Gamma_{\mathrm{tb}} \subseteq \Gamma_{\mathrm{r}}$.

Proof. Take any game $(N, v) \in \Gamma_{\mathrm{tb}}$. The zero-normalized value function $v_{0}$ corresponding to $v$ is defined by

$$
v_{0}(C)=v(C)-\sum_{i \in C} v(\{i\}), \quad C \in 2^{N}
$$

It is well-known that $\left(N, v_{0}\right) \in \Gamma_{\mathrm{tb}}$. Using the singletons with weights 1 it follows from the total balancedness of $v_{0}$ that for any $C \in 2^{N}$

$$
0=\sum_{i \in C} v_{0}(\{i\}) \leq v_{0}(C)
$$

Moreover, any $C \in 2^{N}$ partitions $N$ into $C$ and $N \backslash C$, and using weights 1 on $C$ and $N \backslash C$ leads to

$$
v_{0}(C)+v_{0}(N \backslash C) \leq v_{0}(N)
$$

Using Equations (4.5) and (4.6) we obtain that for any $C \in 2^{N}$

$$
0 \leq v_{0}(C) \leq v_{0}(N)
$$


The remainder of the proof is constructive. We specify the risk environment $\left(N, S, p, X^{0}, \rho\right)$ as follows. We introduce a state of nature for all non-empty coalitions of $N$, so $S=2^{n}-1$. We label states of nature by $C, D \in 2^{N} \backslash\{\emptyset\}$. We consider equiprobably outcomes, $p_{1}=\cdots=p_{S}=1 / S$, and we let $\rho$ be the risk measure equal to the 1-expected shortfall (Definition 4.3) with $\delta=1$. For each state of nature $C \in 2^{N} \backslash\{\emptyset\}$, let the row vector $X_{C}^{0}$. be such that

$$
\begin{aligned}
& \left(X_{C, i}^{0}\right)_{i \in C} \text { belongs to the core of }\left(C, v_{0}^{C}\right), \\
& X_{C, i}^{0}=v_{0}(N), \quad i \in N \backslash C .
\end{aligned}
$$

The risk environment $\left(N, S, p, X^{0}, \rho\right)$ induces the game $\left(N, \bar{v}_{0}\right)$. We will show that $\bar{v}_{0}=v_{0}$. By the definition of 1-expected shortfall, we have

$$
\bar{v}_{0}(C)=-\rho\left(X^{0}(C)\right)=\min _{D \in 2^{N} \backslash\{\emptyset\}} X_{D}^{0}(C), \quad C \in 2^{N} .
$$

The definition of a subgame, Equation (4.8), and the efficiency of a core element imply

$$
v_{0}^{C}(C)=v_{0}(C)=X_{C}^{0}(C), \quad C \in 2^{N} \backslash\{\emptyset\}
$$

We show next that

$$
X_{C}^{0}(C) \leq X_{D}^{0}(C), \quad C, D \in 2^{N} \backslash\{\emptyset\}
$$

Indeed, if $D \supseteq C$ then Inequality (4.12) follows from (4.8) as we have for a core element $\left(X_{D, i}^{0}\right)_{i \in D}$ in subgame $\left(D, v_{D}^{0}\right)$ that

$$
X_{C}^{0}(C)=v_{0}(C) \leq X_{D}^{0}(C)
$$

If $D \nsupseteq C$ then one of the components of $\left(X_{D, i}^{0}\right)_{i \in C}$ is $v_{0}(N)$, and using Equation (4.7) Inequality (4.12) follows immediately. Combining Equations (4.11) and (4.12) with Equation (4.10) we obtain that $\bar{v}_{0}=v_{0}$.

By using the matrix of realization vectors $X$ defined by $X_{\cdot i}=X_{\cdot i}^{0}+v(\{i\}) 1^{S}, i \in N$, we obtain a risk environment that induces the game $(N, v)$.

Example 4.16. We illustrate the construction used in the proof of Proposition 4.15 in an example with 3 players. Table 4.1 presents the value function $v$ of a totally balanced game, as well as the 


\begin{tabular}{c|cc}
$C$ & $v(C)$ & $v_{0}(C)$ \\
\hline$\{1\}$ & -10 & 0 \\
$\{2\}$ & 3 & 0 \\
$\{3\}$ & -2 & 0 \\
$\{1,2\}$ & -4 & 3 \\
$\{1,3\}$ & -6 & 6 \\
$\{2,3\}$ & 2 & 1 \\
$\{1,2,3\}$ & -1 & 8
\end{tabular}

Table 4.1: A totally balanced game and its zero-normalized game.

\begin{tabular}{c|ccc|ccc}
$S$ & $X_{\cdot 1}^{0}$ & $X_{\cdot}^{0}$ & $X_{\cdot 3}^{0}$ & $X_{\cdot 1}$ & $X_{\cdot 2}$ & $X_{\cdot 3}$ \\
\hline$\{1\}$ & 0 & 8 & 8 & -10 & 11 & 6 \\
$\{2\}$ & 8 & 0 & 8 & -2 & 3 & 6 \\
$\{3\}$ & 8 & 8 & 0 & -2 & 11 & -2 \\
$\{1,2\}$ & 1 & 2 & 8 & -9 & 5 & 6 \\
$\{1,3\}$ & 2 & 8 & 4 & -8 & 11 & 2 \\
$\{2,3\}$ & 8 & 1 & 0 & -2 & 4 & -2 \\
$\{1,2,3\}$ & 2 & 1 & 5 & -8 & 4 & 3
\end{tabular}

Table 4.2: Payoff matrices for the zero normalized and the original games.

zero-normalized value function $v_{0}$ corresponding to $v$. Note that Inequality (4.7) is satisfied by $v_{0}$.

In Table 4.2 we have specified the matrix of realization vectors $X^{0}$ according to requirements (4.8) and (4.9). For instance, for $C=\{1,2\}$ we have that $\left(X_{\{1,2\}, 1}^{0}, X_{\{1,2\}, 2}^{0}\right)=(1,2)$ is a point in the core of the subgame with players 1 and 2 , and $X_{\{1,2\}, 3}^{0}=8=v_{0}(N)$.

It is easy to check that the risk environment specified by $X^{0}$ and the risk measure equal to the 1-expected shortfall with $\delta=1$ generate $v_{0}$.

To generate the value function $v$, we transform $X^{0}$ into $X$ by specifying $X_{\cdot i}=X_{\cdot i}^{0}+v(\{i\}) 1^{S}$ for all $i \in N$. The risk environment corresponding to $X$ and the risk measure equal to the 1 -expected shortfall with $\delta=1$ can be verified to induce the game $(N, v)$.

Note that in our constructive proof the statement of Proposition 4.15 is strengthened in the sense that the family of games induced by risk environments with $S \leq 2^{n}-1$ and the risk measure equal to the 1 -expected shortfall with $\delta=1$ coincides with the family of totally balanced games with $n$ players, that is any totally balanced game can be generated by a properly specified risk environment with the 1 -expected shortfall and $2^{n}-1$ states of nature. From Propositions 4.14 
and 4.15 we derive the following theorem.

Theorem 4.17. The class of risk allocation games coincides with the class of totally balanced games, $\Gamma_{\mathrm{r}}=\Gamma_{\mathrm{tb}}$.

Kalai and Zemel (1982b) use a similar construction to show that a game is totally balanced if and only if it is the minimum game of a finite collection of additive games. A game $(N, v)$ is called additive if there exists a set of real numbers $b_{1}, \ldots, b_{n}$ such that for every $C \in 2^{N}$, $v(C)=\sum_{i \in C} b_{i}$. For a finite collection of games $\left\{v_{t}\right\}_{t \in T}$ the minimum game is defined by $\left(\min v_{t}\right)(C)=\min _{t \in T} v_{t}(C)$. It is easy to see that the totally balanced game $v$ in Table 4.1 is equal to the minimum game of the collection of additive games generated by $X_{C}, C \in 2^{N} \backslash\{\emptyset\}$, in Table 4.2.

\subsubsection{Linear Programming Results}

Consider a totally balanced game $(N, v) \in \Gamma_{\mathrm{tb}}$. Throughout the subsection, we choose $S=$ $2^{n}-1, p_{1}=\cdots=p_{S}=1 / S$, and $\rho$ the risk measure equal to 1 -expected shortfall with $\delta=1$, just like in Proposition 4.15. Whenever we write $v$ is generated by a matrix of realization vectors $X$ we mean that the risk allocation game induced by the risk environment $(N, S, p, X, \rho)$ equals $(N, v)$.

In the proof of Proposition 4.15 the matrix of realization vectors $X$ generating $v$ was constructed using the core requirement ${ }^{3}$ (4.8): for every $C \in 2^{N} \backslash\{\emptyset\}$

$$
\left(X_{C, i}\right)_{i \in C} \text { belongs to the core of }\left(C, v^{C}\right) .
$$

The other elements of $X$ were chosen to be sufficiently large.

Let a game $(N, v) \in \Gamma$ be given. We develop a linear programming problem such that the optimal value of the objective function will exceed $\sum_{C \in 2^{N}} v(C)$ if $(N, v) \in \Gamma \backslash \Gamma_{\mathrm{tb}}$ and is equal to $\sum_{C \in 2^{N}} v(C)$ whenever the game is totally balanced. Moreover, in the latter case the matrices derived from the linear program's optimal solutions generate $v$.

To do so, given a matrix $X$ we define the vector $\hat{X} \in \mathbb{R}^{S n}$ by juxtaposing the rows of $X \in \mathbb{R}^{S \times n}$, that is $\hat{X}=\left(X_{1}, X_{2 .}, \ldots, X_{S}\right)^{\top} \in \mathbb{R}^{S n}$, and the reverse operation transforms a vector $\hat{X} \in \mathbb{R}^{S n}$ into a matrix $X \in \mathbb{R}^{S \times n}$. We will use the notations $\hat{X}$ and $X$ interchangeably.

\footnotetext{
${ }^{3}$ There we had a zero normalized game, but it is easy to see that after renormalizing the core requirement is still satisfied.
} 
Let $0^{n}=(0,0, \ldots, 0) \in \mathbb{R}^{1 \times n}$ be the $n$-dimensional row vector of zeros. For every $C \in 2^{N} \backslash\{\emptyset\}$, we define the matrices

$$
A(C)=\left(\begin{array}{cccc}
a(C)^{\top} & 0^{n} & & 0^{n} \\
0^{n} & a(C)^{\top} & & 0^{n} \\
& & \ddots & \vdots \\
0^{n} & 0^{n} & \cdots & a(C)^{\top}
\end{array}\right) \in \mathbb{R}^{S \times S n}
$$

containing the membership vector $a(C)$ transposed along the "diagonal" and $0^{n}$ otherwise.

A matrix $X \in \mathbb{R}^{S \times n}$ generates $v$ if and only if for every $C \in 2^{N} \backslash\{\emptyset\}$

$$
v(C)=\min _{D \in 2^{N} \backslash\{\emptyset\}} X_{D}(C) .
$$

Equation (4.16) can be rewritten as

$$
v(C)=\min _{D \in 2^{N}} A_{D \cdot}(C) \hat{X}
$$

where $A_{D \cdot}(C)$ denotes the $D$-th row of $A(C)$. It follows from Equation (4.17) that $X$ generates $v$ if and only if for every $C \in 2^{N} \backslash\{\emptyset\}$

$$
A(C) \hat{X} \geq v(C) 1^{S}
$$

where for each $C$ at least one inequality holds with equality.

We introduce some additional notation. Let

$$
\begin{aligned}
& E=\left(a(\{1\})^{\top}, a(\{2\})^{\top}, \ldots, a(N)^{\top}\right) \in \mathbb{R}^{1 \times S n}, \\
& V=\left(\begin{array}{c}
v(\{1\}) 1^{S} \\
v(\{2\}) 1^{S} \\
\vdots \\
v(N) 1^{S}
\end{array}\right) \in \mathbb{R}^{S^{2}},
\end{aligned}
$$

and

$$
A=\left(\begin{array}{c}
A(\{1\}) \\
A(\{2\}) \\
\vdots \\
A(N)
\end{array}\right) \in \mathbb{R}^{S^{2} \times S n}
$$

Consider the linear programming problem $\left(\mathrm{P}_{v}\right)$ :

$$
\begin{array}{rrl}
\min E \hat{X} & & \\
\text { s.t. } & A \hat{X} & \geq V \\
& \hat{X} & \in \mathbb{R}^{S n}
\end{array}
$$


The objective function of $\left(\mathrm{P}_{v}\right)$ captures the constructive proof of Proposition 4.15, as it is minimizing exactly the sum of those elements of $\hat{X}$ which are used in the core requirement (4.14). Using Equation (4.18) it can be seen that the feasibility constraints in the linear program are the necessary requirements for $v$ to be generated by a feasible solution.

The set of optimal solutions of $\left(\mathrm{P}_{v}\right)$ is non-empty, since $\hat{X}=(k, \ldots, k) \in \mathbb{R}^{S n}$ is a feasible solution, where $k=\max _{C \in 2^{N}} v(C)$ and the set of feasible solutions is bounded from below. We denote the set of optimal solutions of $\left(\mathrm{P}_{v}\right)$ by $\mathcal{X}_{v}^{*}$ and a particular optimal solution by $\hat{X}^{*} \in \mathbb{R}^{S n}$.

Proposition 4.18. Consider a game $(N, v) \in \Gamma$ and an optimal solution $\hat{X}^{*} \in \mathcal{X}_{v}^{*}$ of $\left(\mathrm{P}_{v}\right)$. The optimal value of the objective function $E \hat{X}^{*}$ equals $\sum_{C \in 2^{N}} v(C)$ if and only if $v$ is generated by $X^{*}$.

\section{Proof.}

$(\Rightarrow)$ By the feasibility constraints it holds that

$$
A_{D^{\cdot}}(C) \hat{X}^{*} \geq v(C), \quad C \in 2^{N} \backslash\{\emptyset\}, D \in 2^{N} \backslash\{\emptyset\}
$$

Since by assumption $E \hat{X}^{*}=\sum_{C \in 2^{N}} v(C)$, it follows that

$$
A_{C \cdot}(C) \hat{X}^{*}=v(C), \quad C \in 2^{N} \backslash\{\emptyset\}
$$

It follows by Inequality (4.21) and Equation (4.22) that $v$ is generated by $X^{*}$.

$(\Leftarrow)$ We use a proof by contradiction. Suppose $E \hat{X}^{*} \neq \sum_{C \in 2^{N}} v(C)$. By the feasibility constraints it holds that

$$
E \hat{X}^{*}>\sum_{C \in 2^{N}} v(C) .
$$

Note that $\min _{D \in 2^{N} \backslash\{\emptyset\}} X_{D}^{*}(C)$ is attained in row $C$ of $X^{*}$, since otherwise we could decrease the objective function by substituting the row attaining the minimum for row $C$. Combining this with Equation (4.23) we obtain that there exists a coalition $C \in 2^{N}$ such that

$$
\min _{D \in 2^{N} \backslash\{\emptyset\}} X_{D}^{*}(C)>v(C)
$$

which implies that $v$ is not generated by $X^{*}$, a contradiction. 
Take any matrix of realization vectors $X \in \mathbb{R}^{z \times n}$, where $z$ is a strictly positive integer. Let $Y(X) \in \mathbb{R}^{\left(2^{n}-1\right) \times n}$ denote a matrix in which for all $C \in 2^{N} \backslash\{\emptyset\}$ we have that $Y_{C} .(X)=X_{k}$, where $k \in \arg \min _{k \in\{1,2, \ldots, z\}} X_{k}(C)$. The following proposition claims that the realization matrix $X$ generates $v$ if and only if $\hat{Y}(X)$ is an optimal solution of $\left(\mathrm{P}_{v}\right)$.

Proposition 4.19. Consider a game $(N, v) \in \Gamma_{\mathrm{tb}}$. The matrix of realization vectors $X \in \mathbb{R}^{z \times n}$ generates $v$ if and only if $\hat{Y}(X) \in \mathcal{X}_{v}^{*}$.

\section{Proof.}

$(\Rightarrow)$ Since $X$ generates $v$, for all $C \in 2^{N} \backslash\{\emptyset\}$ there exists a state of nature $s(C)$ such that

$$
\begin{aligned}
X_{s(C)}(C) & =v(C), \\
X_{s}(C) & \geq v(C), \quad s \in\{1, \ldots, S\} .
\end{aligned}
$$

It follows that $\hat{Y}(X)$ is a feasible and optimal solution of $\left(\mathrm{P}_{v}\right)$.

$(\Leftarrow)$ Since the game $(N, v)$ is totally balanced, according to Theorem $4.17 v$ is generated by some matrix of realization vectors. By Proposition 4.18, it follows that $v$ is generated by all elements of $\mathcal{X}_{v}^{*}$, so $Y(X)$ generates $v$, and by construction $X$ generates $v$.

The following result shows that any matrix of realization vectors $X$ that generates $v$ satisfies (4.14).

Proposition 4.20. Consider a game $(N, v) \in \Gamma_{\mathrm{tb}}$. Any optimal solution of $\left(P_{v}\right) \hat{X}^{*} \in \mathcal{X}_{v}^{*}$ satisfies the core requirement (4.14).

Proof. Take any $\hat{X}^{*} \in \mathcal{X}_{v}^{*}$. Since by Proposition 4.15 all totally balanced games can be generated, we know by Proposition 4.18 that $E \hat{X}^{*}=\sum_{C \in 2^{N}} v(C)$. For every $C \in 2^{N} \backslash\{\emptyset\}$, feasibility requires that $A_{C \cdot}(C) \hat{X}^{*} \geq v(C)$, so

$$
A_{C \cdot}(C) \hat{X}^{*}=v(C)
$$

The equalities in (4.25) together with the feasibility constraints imply that the rows of $X^{*}$ contain core allocations of the respective subgames. 
Propositions 4.19 and 4.20 imply that if a game is generated by $X \in \mathbb{R}^{z \times n}$, then $Y(X)$ satisfies the core requirement (4.14). Thus to generate a given totally balanced game the rows of the matrix of realization vectors can be permutated and some of them can be combined, but essentially the core requirement (4.14) is satisfied in all of them.

\subsection{Exactness}

In this section we show that if there is no aggregate uncertainty in a risk environment, then the induced risk allocation game is an exact game, and conversely all exact games can be generated by a properly specified risk environment with no aggregate uncertainty. Proposition 4.21 claims that risk allocation games with no aggregate uncertainty are exact.

Proposition 4.21. All games with $(N, v) \in \Gamma_{\text {rnau }}$ are exact, $\Gamma_{\text {rnau }} \subseteq \Gamma_{\mathrm{e}}$.

Proof. Consider the risk environment $(N, S, p, X, \rho)$, where $X$ has no aggregate uncertainty. We show that the induced risk allocation game is totally balanced and overbalanced (Definition 4.8), so exact by Theorem 4.9. Total balancedness follows from Proposition 4.14. For overbalancedness take any vector $\left(\lambda^{C}\right)_{C \in 2^{N}} \in \mathbb{R}_{+}^{2^{N}}$ such that $\sum_{C \in 2^{N} \backslash\{D, N\}} \lambda^{C} a(C)=a(N)+\lambda^{D} a(D)$. Then by Equation (4.3), the positive homogeneity, and subadditivity of $\rho$ we have that

$$
\begin{aligned}
\sum_{C \in 2^{N} \backslash\{D, N\}} \lambda^{C} v(C) & =-\sum_{C \in 2^{N} \backslash\{D, N\}} \rho\left(\sum_{i \in C} \lambda^{C} X_{\cdot i}\right) \\
& \leq-\rho\left(\sum_{C \in 2^{N} \backslash\{D, N\}}\left(\sum_{i \in C} \lambda^{C} X_{\cdot i}\right)\right) \\
& =-\rho\left(\sum_{i \in N}\left(\sum_{C \ni i, C \in 2^{N} \backslash\{D, N\}} \lambda^{C} X_{\cdot i}\right)\right) \\
& =-\rho\left(\sum_{i \in N} X_{\cdot i}+\lambda^{D} \sum_{i \in D} X_{\cdot i}\right),
\end{aligned}
$$

where the last two lines follow from rearranging the summation and using the fact that we have an overbalanced vector of weights, thus if $i \in D$ then $\sum_{C \ni i, C \in 2^{N} \backslash\{D, N\}} \lambda^{C}=1+\lambda^{D}$, and if $i \notin D$ then $\sum_{C \ni i, C \in 2^{N} \backslash\{D, N\}} \lambda^{C}=1$. Using translation invariance and positive homogeneity, 
Inequality (4.26) can be rewritten as

$$
\begin{aligned}
\sum_{C \in 2^{N} \backslash\{D, N\}} \lambda^{C} v(C) & \leq-\rho\left(\sum_{i \in N} X_{\cdot i}+\lambda^{D} \sum_{i \in D} X_{\cdot i}\right) \\
& =-\rho(X(N))-\rho\left(\lambda^{D} X(D)\right) \\
& =-\rho(X(N))-\lambda^{D} \rho(X(D)) \\
& =v(N)+\lambda^{D} v(D),
\end{aligned}
$$

thus we have an overbalanced game.

Proposition 4.22 shows that each exact game is generated by some risk environment with no aggregate uncertainty.

Proposition 4.22. Each game $(N, v) \in \Gamma_{e}$ is induced by some risk environment $(N, S, p, X, \rho)$ such that $X$ has no aggregate uncertainty, $\Gamma_{\mathrm{e}} \subseteq \Gamma_{\text {rnau. }}$.

Proof. Consider the exact game $(N, v) \in \Gamma_{\mathrm{e}}$. We specify the risk environment $(N, S, p, X, \rho)$ as follows. We introduce a state of nature for all proper non-empty subcoalitions of $N$, thus $S=2^{n}-2$. Let $p_{1}=\cdots=p_{S}=1 / S$, and let $\rho$ be the 1 -expected shortfall with $\delta=1$. For all $C \in 2^{N} \backslash\{\emptyset\}$ there exist a core element $x_{C}$ such that $x_{C}(C)=v(C)$ since $(N, v)$ is exact. Construct $X \in \mathbb{R}^{S \times n}$ as follows. We define, for all $C \in 2^{N} \backslash\{\emptyset, N\}, X_{C}=x_{C}$. Since $x_{C}$ is a core element, it holds that $X_{C .}(N)=v(N)$, thus $X$ has no aggregate uncertainty. We denote the game induced by the risk environment by $\bar{v}$. Now we have for every $C \in 2^{N} \backslash\{\emptyset\}$ that

$$
\bar{v}(C)=\min _{D \in 2^{N} \backslash\{\emptyset, N\}} X_{D}(C)=v(C)
$$

thus $\bar{v}=v$.

Note that in the proof of Proposition 4.22 the sum of the entries in each row of $X$ is equal to $v(N)$. That is why we need only $2^{n}-2$ states of nature.

Combining Propositions 4.21 and 4.22 we have the following theorem.

Theorem 4.23. The class of risk allocation games with no aggregate uncertainty coincides with the class of exact games, $\Gamma_{\text {rnau }}=\Gamma_{\mathrm{e}}$. 
Biswas, Parthasarathy, Potters, and Voorneveld (1999) show that a game is convex if and only if any of its subgame is exact. We have provided an alternative proof in Theorem 3.18. This result implies directly that if there are less than four players, then the class of exact games coincides with the class of convex games. Using this observation Theorem 4.23 can be reformulated as follows.

Theorem 4.24. Let $n \in \mathbb{N}$ be such that $n<4$. Then the class of risk allocation games with $n$ portfolios and no aggregate uncertainty coincides with the class of convex games with n players, $\Gamma_{\text {rnau }}=\Gamma_{\text {c. }}$

Theorem 4.24 is illustrated by the following example.

Example 4.25. In this example we show how a 3-player convex game is generated by a risk allocation game with no aggregate uncertainty. Note that the game in Table 4.1 of Example 4.16 is not convex since $v(\{1,2\})+v(\{1,3\})=-4-6=-10>v(\{1\})+v(\{1,2,3\})=-10-1=$ -11 . However, by changing $v(\{1,2\})$ to -5 we get the convex game displayed in Table 4.3.

\begin{tabular}{c|c}
$C$ & $v(C)$ \\
\hline$\{1\}$ & -10 \\
$\{2\}$ & 3 \\
$\{3\}$ & -2 \\
$\{1,2\}$ & -5 \\
$\{1,3\}$ & -6 \\
$\{2,3\}$ & 2 \\
$\{1,2,3\}$ & -1
\end{tabular}

Table 4.3: The value function of a convex game $v$.

\begin{tabular}{c|cccc}
$S$ & $X_{\cdot 1}$ & $X_{\cdot 2}$ & $X_{\cdot 3}$ & $\sum_{i \in N} X_{s, i}$ \\
\hline 1 & -3 & 4 & -2 & -1 \\
2 & -7 & 3 & 3 & -1 \\
3 & -10 & 5 & 4 & -1
\end{tabular}

Table 4.4: A matrix of realization vectors generating $v$.

This game is generated by the risk environment with matrix of realization vectors $X$ depicted in Table 4.4 and the risk measure of 1-expected shortfall with $\delta=1$. 
Notice that the rows of $X$ correspond to appropriately chosen marginal contribution vectors. For instance, in the first row of $X$ we have the marginal contribution vector corresponding to the permutation $(3,2,1): v(\{3\})-v(\{\emptyset\})=-2-0=-2, v(\{2,3\})-v(\{2\})=2-(-2)=4$, and $v(\{1,2,3\})-v(\{2,3\})=-1-2=-3$. At any marginal contribution vector, there are $n$ coalitions that exactly receive their value. Thus to generate a convex game fewer states of nature are required than $2^{n}-1$. In the example 3 states of nature suffice. Also note that all rows of $X$ sum up to -1 , since the sum of the marginal contributions is always the value of the grand coalition, and there is no aggregate uncertainty.

Similarly to Proposition 4.19 we can characterize all the risk environments that generate a given exact game.

Proposition 4.26. Consider a game $(N, v) \in \Gamma_{\mathrm{e}}$. The matrix of realization vectors $X \in \mathbb{R}^{z \times n}$ without aggregate uncertainty generates $v$ if and only if $\hat{Y}(X) \in \mathcal{X}_{v}^{*}$.

Proof. Proposition 4.19 characterizes all the matrices that generate a given totally balanced game. Since by Proposition 4.21 only exact games can be generated with matrices satisfying no aggregate uncertainty, the proof is straightforward.

\subsection{Conclusion}

In this chapter we have discussed transferable utility cooperative games derived from a risk environment: risk allocation games. We have shown that the class of risk allocation games coincides with the class of totally balanced games. This result makes sure that a regulator or performance evaluator can always allocate risk in a stable way: there will always be a core element, no matter how the risk environment is changing.

We have also studied the case when the aggregate portfolio has the same payoff in all states of nature. We proved that if there is no aggregate uncertainty then the class of risk allocation games equals the class of exact games, where for each coalition there is a core element such that the coalition gets only its stand-alone value. This means that if there is no aggregate uncertainty, then not necessarily everybody benefits from the diversification effects in a stable allocation of risk. The regulator or performance evaluator has much discretionary power in allocating risk, 
since for each coalition there is always a stable allocation of risk such that the coalition gets its stand-alone value.

We have characterized all the matrices of realization vectors that generate a given totally balanced game or a given exact game. In both cases the vectors derived from the matrices by juxtaposing their rows are related to the optimal solutions of a linear programming problem.

Denault (2001) shows that if a risk allocation game for an arbitrary matrix of realization vectors is convex then the risk measure by which it is induced is necessarily additive, thus the generated risk allocation game is also additive. However, by imposing some structure on the matrix of realization vectors we have proven the following theorem: If there are less than four players and the matrix of realization vectors has no aggregate uncertainty, then the generated risk allocation game is convex, and any convex game can be generated by such a risk environment. 


\section{Chapter 5}

\section{Convex and Exact Games with Non-transferable Utility}

In this chapter we generalize exactness to games with non-transferable utility (NTU). In an exact NTU game for each coalition there is a core allocation on the boundary of its payoff set, meaning that this coalition does not necessarily benefit from the gains of forming the grand coalition in an allocation which is robust against all coalitional deviations. We show that each of ordinal, coalition merge, individual merge and marginal convexity can be unified under NTU exactness. Finally, we relate the classes of $\Pi$-balanced, totally $\Pi$-balanced, NTU exact, totally NTU exact, ordinally convex, coalition merge convex, individual merge convex and marginal convex games to one another.

\subsection{Introduction}

Convex cooperative games with transferable utility (TU) introduced by Shapley (1971) arise from a wide range of applications. Airport games (Littlechild and Owen, 1973), bankruptcy games (Aumann and Maschler, 1985), sequencing games (Curiel, Pederzoli, and Tijs, 1989) and standard tree games (Granot, Maschler, Owen, and Zhu, 1996) are all convex.

Convex TU games are exact (Schmeidler, 1972). In an exact game for each coalition there is a core allocation such that the coalition only gets its stand-alone value. In Chapter 4 we have shown that the class of exact TU games equals the class of risk allocation games with no aggregate uncertainty. A risk allocation game is a TU game derived from a coherent measure of risk (Artzner, Delbaen, Eber, and Heath, 1999) and the realization vectors of some individual portfolios. Usually the risk of the aggregate portfolio is low compared to the risk involved in the 
individual portfolios. As an extreme case, no aggregate uncertainty refers to the case when the value of the aggregate portfolio is constant over all states of nature.

Vilkov (1977) and Sharkey (1981) have extended convexity to games with non-transferable utility (NTU) to define ordinal and cardinal convexity, respectively. Hendrickx, Borm, and Timmer (2002) analyze coalition merge convexity, individual merge convexity, and marginal convexity in an NTU setting.

Ordinally convex NTU games have numerous applications. Peleg (1984) transforms a social choice situation with a convex effectivity function into an NTU game which is ordinally convex. Demange (1987) provides two examples: a model of public good, and a production economy with increasing returns to scale; Masuzawa (2003) adds $N$-person prisoners' dilemma games and oligopoly models to this class.

Convex NTU games are important classes of games, but unfortunately the aforementioned five classes of NTU convex games do not coincide in general. The only general result (Theorem 5.14 ) is that coalition merge convexity implies individual merge convexity, and individual merge convexity implies marginal convexity. It is natural to seek a result that is analogous to convex TU games being exact.

In this chapter we generalize exactness to the NTU setting. In an exact NTU game for each coalition there is a core element on the boundary of its payoff set, meaning that this coalition does not necessarily benefit from the gains of forming the grand coalition in an allocation which is robust against all coalitional deviations. We show that each of ordinal, coalition merge, individual merge and marginal convexity implies NTU exactness.

The structure of the chapter is as follows. We start with the notation and the necessary definitions for TU and NTU games. In Section 5.3 we define NTU exactness and show that an NTU game satisfying any of ordinal, coalition merge, individual merge and marginal convexity is NTU exact. In Section 5.4 we conclude by relating the various classes of NTU games to one another.

\subsection{Notation, Definitions, Existing Results}

Let $N=\{1, \ldots, n\}$ denote the finite set of players, $2^{N}=\{C \mid C \subseteq N\}$ is the power set of $N$, $\mathcal{N}=2^{N} \backslash\{\emptyset\}$ is the collection of coalitions, the non-empty subsets of $N$. Let $\mathbb{R}$ denote the set of all real numbers. $\mathbb{R}^{N}$ is the $n$-dimensional Euclidean space generated by the set of players. 
An element of $\mathbb{R}^{N}$ is denoted by a vector $x=\left(x_{i}\right)_{i \in N}$. For a coalition $C \in \mathcal{N}$, let $x^{C}=\left(x_{i}\right)_{i \in C}$ denote the restriction of $x$ on $C$. For $x, y \in \mathbb{R}^{N}, y \geq x$ denotes $y_{i} \geq x_{i}$ for all $i \in N$, and $y \gg x$ denotes $y_{i}>x_{i}$ for all $i \in N$.

Let $\Delta^{N}$ denote the unit simplex in $\mathbb{R}^{N}, \Delta^{N}=\left\{x \in \mathbb{R}^{N} \mid x_{i} \geq 0, \sum_{i \in N} x_{i}=1\right\}$. For every $C \in$ $\mathcal{N}$ define the set $\Delta^{C}=\left\{x \in \Delta^{N} \mid \sum_{i \in C} x_{i}=1\right\}$, and let $\Delta$ be a Cartesian product of $\Delta^{C}$ over all $C \in \mathcal{N}$. Let $\Delta^{\mathcal{N}}$ denote the unit simplex in $\mathbb{R}^{\mathcal{N}}, \Delta^{\mathcal{N}}=\left\{\lambda \in \mathbb{R}^{\mathcal{N}} \mid \lambda^{C} \geq 0, \sum_{C \in \mathcal{N}} \lambda^{C}=1\right\}$. For a set $A \subseteq \mathbb{R}^{N}$, the symbols cl $A, \partial A$ and int $A$ denote, respectively, the closure, the boundary and the interior of $A$. For $x \in \mathbb{R}^{N}, x \in \operatorname{cl} A$ if there exists a sequence $\left(x^{k}\right)_{k \in \mathbb{N}}$ with $x^{k} \in A$ for all $k \in \mathbb{N}$ and $\left(x^{k}\right)_{k \in \mathbb{N}} \rightarrow x ; x \in \partial A$ if $x \in \operatorname{cl} A \cap \mathrm{cl}\left(\mathbb{R}^{N} \backslash A\right)$; and $x \in \operatorname{int} A$ if $x \in A \backslash \partial A$.

\subsubsection{Transferable Utility Games}

A value function $v: 2^{N} \rightarrow \mathbb{R}$ satisfying $v(\emptyset)=0$ gives rise to a cooperative game with transferable utility (TU game, for short) $(N, v)$. Let $\Gamma^{\mathrm{TU}}$ denote the set of TU games with $n$ players. An allocation is a vector $x \in \mathbb{R}^{N}$, where $x_{i}$ is the payoff of player $i \in N$. For a coalition $C \in \mathcal{N}$, let $x(C)=\sum_{i \in C} x_{i}$. An allocation $x \in \mathbb{R}^{N}$ is called efficient if $x(N)=v(N)$, individually rational if $x_{i} \geq v(\{i\})$ for all $i \in N$, and coalitionally rational if $x(C) \geq v(C)$ for all $C \in \mathcal{N}$. The core is the set of efficient and coalitionally rational allocations.

Schmeidler (1972) introduces exact TU games.

Definition 5.1. A TU game $(N, v)$ is exact if for each $C \in 2^{N}$ there exists a core allocation $x$ such that $x(C)=v(C)$.

Let $\Gamma_{\mathrm{e}}^{\mathrm{TU}}$ denote the class of exact TU games with $n$ players. Convex TU games (Shapley, 1971) can be defined and characterized as follows.

Definition 5.2. A TU game $(N, v)$ is convex if it satisfies the following three equivalent conditions:

$$
\begin{array}{r}
\forall S, T \in 2^{N}: v(S)+v(T) \leq v(S \cup T)+v(S \cap T), \\
\forall U \in 2^{N} ; \forall S \subsetneq T \subseteq N \backslash U: v(S \cup U)-v(S) \leq v(T \cup U)-v(T), \\
\forall i \in N ; \forall S \subsetneq T \subseteq N \backslash\{i\}: v(S \cup\{i\})-v(S) \leq v(T \cup\{i\})-v(T) .
\end{array}
$$

Let $\Gamma_{\mathrm{c}}^{\mathrm{TU}}$ denote the class of convex TU games with $n$ players. 
A permutation of the players in $N$ is a bijection $\sigma:\{1, \ldots, n\} \mapsto N$, where $\sigma(i)$ denotes which player in $N$ is at position $i$, and $\sigma^{-1}(i)$ denotes the position of player $i$. Let $G^{n}$ denote the set of all permutations on $N$. For a permutation $\sigma \in G^{n}, P_{i}^{\sigma}=\left\{j \in N \mid \sigma^{-1}(j)<\sigma^{-1}(i)\right\}$ denotes the coalition of players which precede $i$ with respect to the order $\sigma$. In a permutation $\sigma \in G^{n}$, $m_{i}^{\sigma}(v)=v\left(P_{i}^{\sigma} \cup\{i\}\right)-v\left(P_{i}^{\sigma}\right)$ denotes the marginal contribution of player $i$ to the preceding players, and $m^{\sigma}(v)=\left(m_{1}^{\sigma}, m_{2}^{\sigma}, \ldots, m_{n}^{\sigma}\right)$ is the vector of marginal contributions. Shapley (1971) and Ichiishi (1981) characterize convex TU games as follows.

Theorem 5.3. The TU game $(N, v)$ is convex if and only if $m^{\sigma}(v)$ belongs to the core of $(N, v)$ for all $\sigma \in G^{n}$.

Theorem 5.3 implies directly the following theorem.

Theorem 5.4. If a TU game $(N, v)$ is convex, then it is exact, $\Gamma_{\mathrm{c}}^{\mathrm{TU}} \subseteq \Gamma_{\mathrm{e}}^{\mathrm{TU}}$.

For a TU game $(N, v)$ and a coalition $C \in \mathcal{N}$ the subgame $\left(C, v^{C}\right)$ is obtained by restricting $v$ to subsets of $C$. Following Biswas, Parthasarathy, Potters, and Voorneveld (1999), we define totally exact TU games.

Definition 5.5. A TU game $(N, v)$ is totally exact if for every $C \in \mathcal{N}$ its subgame $\left(C, v^{C}\right)$ is exact.

Let $\Gamma_{\mathrm{te}}^{\mathrm{TU}}$ denote the class of totally exact TU games with $n$ players. Biswas, Parthasarathy, Potters, and Voorneveld (1999) show the following theorem.

Theorem 5.6. A TU game is totally exact if and only if it is convex, $\Gamma_{\mathrm{te}}^{\mathrm{TU}}=\Gamma_{\mathrm{c}}^{\mathrm{TU}}$. 


\subsubsection{Non-transferable Utility Games}

A cooperative game with non-transferable utility (NTU game, for short) $(N, V)$ is a family of sets $V=\langle V(S)\rangle_{S \in 2^{N}}$ satisfying the following assumptions:

$V(\emptyset)=\emptyset$

$V(S)=V_{\mathrm{p}}(S) \times \mathbb{R}^{N \backslash S}$, where $V_{\mathrm{p}}(S) \subseteq \mathbb{R}^{S}$, for all $S \in \mathcal{N}$,

$0 \in V(S)$ for all $S \in \mathcal{N}$,

$V(N)$ is closed,

comprehensiveness: if $x \in V(S), y \in \mathbb{R}^{N}, y^{S} \leq x^{S}$, then $y \in V(S)$,

the sets $V_{\mathrm{p}}^{+}(S)=\left\{x^{S} \in \mathbb{R}_{+}^{S} \mid x \in V(S)\right\}$ are bounded for all $S \in \mathcal{N}$.

Let $\Gamma^{\mathrm{NTU}}$ denote the set of NTU games with $n$ players.

The core of an NTU game $(N, V), C(V)$ consists of those elements $x \in V(N)$ for which it holds that there exist no $S \in \mathcal{N}$ and no $y \in V(S)$ such that $x^{S} \ll y^{S}$, which by comprehensiveness is equivalent to $x \notin$ int $V(S)$ for any $S \in \mathcal{N}$. Therefore,

$$
C(V)=V(N) \backslash \bigcup_{S \in \mathcal{N}} \text { int } V(S) .
$$

Predtetchinski and Herings (2004) provide the following balancedness condition for NTU games.

Definition 5.7. Consider a convex-valued correspondence $\Pi: \mathbb{R}^{N} \mapsto \Delta$ with a closed graph. The NTU game $(N, V)$ is $\Pi$-balanced provided that the following condition is satisfied: If $x \in$ $\mathbb{R}^{N}, \pi \in \Pi(x)$, and $\lambda \in \Delta^{\mathcal{N}}$ are such that

$$
\begin{aligned}
& x \in \bigcap_{S \in \mathcal{N}, \lambda^{S}>0} V(S), \\
& \pi^{N}=\sum_{S \in \mathcal{N}} \lambda^{S} \pi^{S}
\end{aligned}
$$

then $x \in V(N)$.

$\Pi$-balancedness is a necessary and sufficient condition for the core in a non-transferable utility game to be non-empty. Let $\Gamma_{\Pi-\mathrm{b}}^{\mathrm{NTU}}$ denote the class of $\Pi$-balanced NTU games with $n$ players. For an NTU game $(N, V)$ and a coalition $S \in \mathcal{N}$ a subgame $\left(S, V^{S}\right)$ is obtained by restricting $V$ to subsets of $S$. Let $\Gamma_{\mathrm{t}-\Pi-\mathrm{b}}^{\mathrm{NTU}}$ denote the class of totally $\Pi$-balanced NTU games with $n$ players, having a non-empty core in each of their subgames. 
NTU convex games have been defined in five ways.

Definition 5.8. (Vilkov, 1977) An NTU game $(N, V)$ is ordinally convex if for all $S, T \in \mathcal{N}$ we have $V(S) \cap V(T) \subseteq V(S \cap T) \cup V(S \cup T)$.

Let $\Gamma_{\mathrm{oc}}^{\mathrm{NTU}}$ denote the class of ordinally convex NTU games with $n$ players. For $S \in 2^{N}$ let $V^{\circ}(S)=\left\{x \in V(S) \mid x_{i}=0\right.$ for all $\left.i \in N \backslash S\right\}$. Note that $V^{\circ}(S)=V_{\mathrm{p}}(S) \times\left\{0^{N \backslash S}\right\}$, for all $S \in \mathcal{N}$

Definition 5.9. (Sharkey, 1981) An NTU game $(N, V)$ is cardinally convex if for all $S, T \in \mathcal{N}$ we have $V^{\circ}(S)+V^{\circ}(T) \subseteq V^{\circ}(S \cap T)+V^{\circ}(S \cup T)$.

Let $\Gamma_{\mathrm{cc}}^{\mathrm{NTU}}$ denote the class of cardinally convex NTU games with $n$ players.

To discuss the three marginalistic interpretations of NTU convexity (coalition merge, individual merge and marginal convexity) introduced by Hendrickx, Borm, and Timmer (2002), we need the following definitions. Note that the definitions are modified compared to Hendrickx, Borm, and Timmer (2002), since we use Assumptions (5.4)-(5.9) whereas they assume that $V(S) \subseteq \mathbb{R}^{S}$ and $V(S)$ is closed for all $S \in \mathcal{N}$; they do not define $V(\emptyset)$; and they assume zero normalization and monotonicity.

An NTU game $(N, V)$ is zero normalized if $V(\{i\})=\left\{x \in \mathbb{R}^{N}: x_{i} \leq 0\right\}$ for all $i \in N$; it is monotonic if for all $S \subseteq T \subseteq N$ and for all $x \in V(S)$ there exists a $y \in V(T)$ such that $y^{S} \geq x^{S}$. The set of weakly Pareto efficient allocations for coalition $S \in \mathcal{N}$ is defined by $\operatorname{WP}(S)=\{x \in$ $\left.V(S) \mid \nexists y \in V(S): y^{S} \gg x^{S}\right\}$. Let $\alpha_{i}=\sup \left\{x_{i} \mid x \in V(\{i\})\right\}, i \in N$. The set of individually rational allocations for coalition $S \in \mathcal{N}$ is defined by $\operatorname{IR}(S)=\left\{x \in V(S) \mid \forall i \in S: x_{i} \geq \alpha_{i}\right\}$. An NTU game $(N, V)$ is superadditive if for all coalitions $S, T \in \mathcal{N}$ such that $S \cap T=\emptyset$ we have $V(S) \cap V(T) \subseteq V(S \cup T)$; it is individually superadditive if for all $i \in N$ and for all $S \subseteq \mathcal{N} \backslash\{i\}$ we have $V(S) \cap V(\{i\}) \subseteq V(S \cup\{i\})$

Definition 5.10. Consider an NTU game $(N, V)$ and a permutation $\sigma \in G^{n}$. The vector of marginal contributions $M^{\sigma}(V)$ is defined by

$$
\begin{array}{r}
M_{\sigma(j)}^{\sigma}(V)=\sup \left\{y_{\sigma(j)} \mid y \in V(\sigma(1), \ldots, \sigma(j)),\right. \\
\left.\forall i \in\{1, \ldots, j-1\}: y_{\sigma(i)} \geq M_{\sigma(i)}^{\sigma}(V)\right\}
\end{array}
$$

for all $j \in\{1, \ldots, n\}$. 
Note that in Definition 5.10 we use supremum, since we do not assume $V(S)$ to be closed for all $S \in \mathcal{N}$, and we also do not assume monotonicity (we define $\sup (\emptyset)=-\infty$ ).

Equation (5.2) in Definition 5.2 says that for any coalition $U$, the marginal contribution to a larger coalition is larger. The same idea in the NTU setting is formulated as follows.

Definition 5.11. An NTU game $(N, V)$ is coalition merge convex if for all $S, T \in 2^{N}, U \in \mathcal{N}$ such that $S \subsetneq T \subseteq N \backslash U$ the following statement is true: For all $x \in \operatorname{WP}(S) \cap \operatorname{IR}(S)$, all $y \in V(T)$, and all $z \in V(S \cup U)$ such that $z^{S} \geq x^{S}$, there exists a $v \in V(T \cup U)$ such that $v^{T} \geq y^{T}$ and $v^{U} \geq z^{U}{ }^{1}$

Let $\Gamma_{\mathrm{cmc}}^{\mathrm{NTU}}$ denote the class of coalition merge convex NTU games with $n$ players. Equation (5.3) in Definition 5.2 says that for any player $i$, the marginal contribution to a larger coalition is larger. The analogous concept in the NTU setting reads as follows.

Definition 5.12. An NTU game $(N, V)$ is individual merge convex if for all $S, T \in 2^{N}, j \in N$ such that $S \subsetneq T \subseteq N \backslash\{j\}$ the following statement is true: For all $x \in \operatorname{WP}(S) \cap \operatorname{IR}(S)$, all $y \in V(T)$, and all $z \in V(S \cup\{j\})$ such that $z^{S} \geq x^{S}$, there exists a $v \in V(T \cup\{j\})$ such that $v^{T} \geq y^{T}$ and $v_{j} \geq z_{j}{ }^{2}$

Let $\Gamma_{\mathrm{imc}}^{\mathrm{NTU}}$ denote the class of individual merge convex NTU games with $n$ players. Theorem 5.3 is also generalized to the NTU setting.

Definition 5.13. An NTU game $(N, V)$ is marginal convex if for all $\sigma \in G^{n}$ we have $M_{\sigma}(V) \in$ $C(V)$.

Let $\Gamma_{\mathrm{mc}}^{\mathrm{NTU}}$ denote the class of marginal convex NTU games with $n$ players. The five notions of NTU convexity are not equivalent in general. Hendrickx, Borm, and Timmer (2002) show that ordinal and cardinal convexity are not related to the other four types of convexity. They also prove the following theorem.

Theorem 5.14. If an NTU game $(N, V)$ is coalition merge convex, then it is individual merge convex, $\Gamma_{\mathrm{cmc}}^{\mathrm{NTU}} \subseteq \Gamma_{\mathrm{imc}}^{\mathrm{NTU}}$. If an NTU game $(N, V)$ is individual merge convex, then it is marginal convex, $\Gamma_{\mathrm{imc}}^{\mathrm{NTU}} \subseteq \Gamma_{\mathrm{mc}}^{\mathrm{NTU}}$.

\footnotetext{
${ }^{1}$ Note that superadditivity was required in the original definition of Hendrickx, Borm, and Timmer (2002). In our setting it is implied by coalition merge convexity using Assumption (5.4), $S=\emptyset$ and comprehensiveness.

${ }^{2}$ Note that individual superadditivity was required in the original definition of Hendrickx, Borm, and Timmer (2002). In our setting it is implied by individual merge convexity using Assumption (5.4), $S=\emptyset$ and comprehensiveness.
} 
Proof. In their proof Hendrickx, Borm, and Timmer (2002) assume zero normalization, monotonicity and $V(S)$ is closed for all $S \in \mathcal{N}$. If a game is not zero normalized, then one can zero normalize it and after the proof normalize back. One can show that monotonicity is implied by individual merge convexity. If $V(S)$ is open one can verify that all the steps of the proof of Hendrickx, Borm, and Timmer (2002) remain valid using Definition 5.10.

To illustrate the subtle differences between the various notions of NTU convexity, consider the following example of an ordinally convex NTU game which is neither cardinal, nor marginal, thus by Theorem 5.14 nor individual merge, nor coalition merge convex.

Example 5.15. (Hendrickx, Borm, and Timmer, 2002, Example 4.1.) Consider the following NTU game with player set $N=\{1,2,3\}$. Let

$$
\begin{aligned}
V(\{i\}) & =\left\{x \in \mathbb{R}^{3} \mid x_{i} \leq 0\right\} \text { for all } i \in N, \\
V(\{1,2\}) & =\left\{x \in \mathbb{R}^{3} \mid x_{1} \leq 0, x_{2} \leq 2\right\}, \\
V(\{1,3\}) & =\left\{x \in \mathbb{R}^{3} \mid x_{1}+x_{3} \leq 1\right\}, \\
V(\{2,3\}) & =\left\{x \in \mathbb{R}^{3} \mid x_{2} \leq 0, x_{3} \leq 0\right\}, \\
V(N) & =\left\{x \in \mathbb{R}^{3} \mid \sum_{i \in N} x_{i} \leq 2\right\} .
\end{aligned}
$$

To show that $(N, V)$ is ordinally convex, let $S, T \in \mathcal{N}$ and let $x \in V(S) \cap V(T)$. If $S \subseteq T$, $T \subseteq S$ or $S \cap T=\emptyset$, then ordinal convexity is easy to check. If $S=\{1,2\}$ and $T=\{1,3\}$, then $x_{1} \leq 0$ and thus $x \in V(S \cap T)$. Otherwise, $\sum_{i \in N} x_{i} \leq 2$, thus $x \in V(S \cup T)$.

Cardinal convexity of $(N, V)$ fails, since $(0,2,0) \in V^{\circ}(\{1,2\})$ and $(0,0,1) \in V^{\circ}(\{1,3\})$, but $(0,2,0)+(0,0,1)=(0,2,1) \notin V^{\circ}(\{1\})+V^{\circ}(N)$.

Marginal convexity of $(N, V)$ is also not satisfied, since the vector of marginal contributions corresponding to $\sigma=(1,2,3), M^{\sigma}(V)=(0,2,0)$ does not belong to the core: coalition $\{1,3\}$ blocks it. Therefore, by Theorem 5.14, $(N, V)$ is neither individual merge, nor coalition merge convex.

We will come back to Example 5.15 in Examples 5.18 and 5.20.

To the best of our knowledge cardinally convex games have no applications, and relatively little is known about them: Sharkey (1981) proves that they have a non-empty core in each of their 
subgames; Hendrickx, Borm, and Timmer (2002) show that for 3-player NTU games they imply all the other NTU convexity notions. We will concentrate in the remaining of the chapter on the other NTU convexity notions.

\subsection{Exact NTU Games}

Theorem 5.4 claims that convex TU games are exact. In this section we generalize exactness to the NTU setting and verify whether each of ordinal, coalition merge, individual merge or marginal convexity implies NTU exactness.

Definition 5.16. An NTU game $(N, V)$ is $N T U$ exact if for each $S \in \mathcal{N}$ there exists a core allocation $x \in C(V)$ such that $x \in \partial V(S)$.

Let $\Gamma_{\mathrm{e}}^{\mathrm{NTU}}$ denote the class of exact NTU games with $n$ players. Every TU game $(N, v)$ with $v(S) \geq 0$ for all $S \in \mathcal{N}$ gives rise to an NTU game $(N, V)$ by defining $V(S)=\{x \in$ $\left.\mathbb{R}^{N} \mid x(S) \leq v(S)\right\}$ for all $S \in \mathcal{N}$. Note that Assumptions (5.4)-(5.9) are satisfied by $(N, V)$. It is a straightforward exercise to verify the following theorem.

Theorem 5.17. A TU game $(N, v)$ is exact if and only if the corresponding $N T U$ game $(N, V)$ is NTU exact.

Note that if an NTU game $(N, V)$ is NTU exact, then each of its subgame has a core element, since by definition for each $S \in \mathcal{N}$ there exists a core allocation $x \in C(V)$ such that $x \in \partial V(S)$, and $x$ cannot be blocked in the subgame $\left(S, V^{S}\right)$ either. Thus exact NTU games are a subset of totally П-balanced games, $\Gamma_{\mathrm{e}}^{\mathrm{NTU}} \subseteq \Gamma_{\mathrm{t}-\Pi-\mathrm{b}}^{\mathrm{NT}}$.

Next, we check whether the NTU game in Example 5.15 is NTU exact.

Example 5.18. (Example 5.15 continued.)

The NTU game $(N, V)$ in Example 5.15 is NTU exact, since $(0,0,2)$ is a core element on the boundary of $V(\{1\}), V(\{2\}), V(\{1,2\}) ;(2,0,0)$ is a core element on the boundary of $V(\{2\})$, $V(\{3\}), V(\{2,3\})$; and $(1,1,0)$ is a core element on the boundary of $V(\{1,3\})$.

If for all $S \in \mathcal{N}$ all core elements of the subgame $\left(S, V^{S}\right)$ could be extended to the core of the original game by increasing the elements outside $S$, then NTU exactness would follow immediately, since core elements of $\left(S, V^{S}\right)$ are on the boundary of $V(S)$. Example 5.15 shows that 
exactness of an ordinally convex NTU game is not so trivial. The core of the subgame related to coalition $\{1,2\}$ is $\left\{x \in \mathbb{R}^{2} \mid x_{1}=0,0 \leq x_{2} \leq 2\right\}$. Note that only some elements in this core can be extended to the core of the original game: $\left\{x \in \mathbb{R}^{2} \mid x_{1}=0,0 \leq x_{2} \leq 1\right\}$, since if $y_{1}=0,1<y_{2} \leq 2, y_{3}=2-y_{2}$, then coalition $\{1,3\}$ blocks allocation $y$ in the original game.

To analyze the NTU exactness of ordinally convex NTU games we need to define the notion of a reduced game. Peleg (1986) uses it when an arbitrary coalition leaves the game, but here we only need to consider one player leaving the grand coalition.

Definition 5.19. Take any NTU game $(N, V), n \geq 2$. Define:

$$
\begin{aligned}
M & =\{1, \ldots, n-1\}=N \backslash\{n\}, m=n-1, \\
\alpha_{n} & =\sup \left\{x_{n} \mid x \in V(\{n\})\right\}, \\
W(S) & =\left\{x \in \mathbb{R}^{M} \mid \exists \beta>\alpha_{n} \text { such that }(x, \beta) \in V(S \cup\{n\})\right\}, S \subseteq M . \\
P(S) & =\left\{x \in \mathbb{R}^{M} \mid(x, \delta) \in V(S), \forall \delta \in \mathbb{R}\right\}, S \subseteq M .
\end{aligned}
$$

Then, the reduced game $(M, U)$ is given by:

$$
U(S)= \begin{cases}\left\{x \in \mathbb{R}^{M} \mid\left(x, \alpha_{n}\right) \in V(N)\right\} & \text { for } S=M, \\ \emptyset & \text { for } S=\emptyset, \\ W(S) \cup P(S) & \text { otherwise. }\end{cases}
$$

Example 5.20. (Example 5.15 continued.)

If player 3 leaves the grand coalition in Example 5.15, then the derived reduced game looks as follows. $U(\{1,2\})=\left\{x \in \mathbb{R}^{2} \mid x_{1}+x_{2} \leq 2\right\}, U(\emptyset)=\emptyset$. Moreover, $W(\{1\})=\left\{x \in \mathbb{R}^{2} \mid x_{1}<\right.$ $1\}, W(\{2\})=\emptyset, P(\{1\})=\left\{x \in \mathbb{R}^{2} \mid x_{1} \leq 0\right\}$ and $P(\{2\})=\left\{x \in \mathbb{R}^{2} \mid x_{2} \leq 0\right\}$ imply that $U(\{1\})=\left\{x \in \mathbb{R}^{2} \mid x_{1}<1\right\}$ and $U(\{2\})=\left\{x \in \mathbb{R}^{2} \mid x_{2} \leq 0\right\}$.

Note that the reduced game is not zero normalized and $U(\{1\})$ is open. Moreover, all the core elements of the reduced game $\left\{x \in \mathbb{R}^{2} \mid x_{1}+x_{2}=2,1 \leq x_{1} \leq 2,0 \leq x_{2} \leq 1\right\}$ can be extended to the core of the original game by setting $x_{3}=\alpha_{n}=0$.

Greenberg (1985) shows the following lemma about reduced games. ${ }^{3}$

Lemma 5.21. (Greenberg, 1985) Consider an ordinally convex NTU game $(N, V)$. Then the reduced game $(M, U)$ is ordinally convex as well.

\footnotetext{
${ }^{3}$ Greenberg (1985) considers the setting when $V(S) \subseteq \mathbb{R}_{+}^{N}$ instead of $V(S) \subseteq \mathbb{R}^{N}$, for all $S \in \mathcal{N}$, but due to Assumptions (5.6) and (5.8) all the arguments can be carried over to this setting.
} 
We show the following theorem.

Theorem 5.22. If an NTU game $(N, V)$ is ordinally convex, then it is exact, $\Gamma_{\mathrm{oc}}^{\mathrm{NTU}} \subseteq \Gamma_{\mathrm{e}}^{\mathrm{NTU}}$.

\section{Proof.}

We proceed by induction. For $n=1$, if an NTU game $(N, V)$ is ordinally convex, then it is exact, since $\max \{x \mid x \in V(N)\}$ is well defined, is on the boundary of $V(N)$ and belongs to the core. Assume that the theorem holds for any game with less than $n$ players. We will show that it also holds for $n$ players. Let $(N, V)$ be an ordinally convex NTU game with $n$ players, $n \geq 2$. Consider the reduced NTU game $(M, U)$ (Definition 5.19).

Since $(M, U)$ is an ordinally convex NTU game with $n-1$ players (Lemma 5.21), we can apply the induction hypothesis. That is, for each $S \in 2^{M} \backslash\{\emptyset\}$ there exists an $x \in C(U)$ such that $x \in \partial U(S)$.

The proof consists of three steps. In Step I we show that $\left(x, \alpha_{n}\right) \in C(V)$ for all $x \in C(U)$; in Step II we establish that for each $S \in 2^{M}$ there exists an $x$, such that $\left(x, \alpha_{n}\right) \in C(V)$ and $\left(x, \alpha_{n}\right) \in \partial V(S \cup\{n\})$; and in Step III we conclude that $(N, V)$ is NTU exact.

Step I. Let $x \in C(U)$. By definition $x \in U(M)$, that is $\left(x, \alpha_{n}\right) \in V(N)$. First, we show that $\left(x, \alpha_{n}\right)$ in $(N, V)$ cannot be blocked by any coalition $T \subsetneq N$. Suppose to the contrary that there exist $\beta>\alpha_{n}, z \gg x$ and $T \subsetneq N$ such that $(z, \beta) \in V(T)$. We consider two cases: $T=M$ or $T \neq M$.

Case 1: $T=M$. Then $(z, \beta) \in V(M)$ and by comprehensiveness for all $\epsilon>0$ we have that $\left(z, \alpha_{n}-\epsilon\right) \in V(M)$. Also, for all $\epsilon>0$ we have that $\left(z, \alpha_{n}-\epsilon\right) \in V(\{n\})$ by the definition of $\alpha_{n}$. Ordinal convexity implies that $V(M) \cap V(\{n\}) \subseteq V(N)$, thus for all $\epsilon>0$ we have that $\left(z, \alpha_{n}-\epsilon\right) \in V(N)$. Since $V(N)$ is closed, $\left(z, \alpha_{n}\right) \in V(N)$, implying that $z \in U(M)$, contradicting $x \in C(U)$.

Case 2: $T \neq M$. If $n \notin T$, then $z \in P(T)$ and hence $T$ would block $x$ in $(M, U)$, contradicting $x \in C(U)$. If $n \in T$, then $T \backslash\{n\} \neq \emptyset$, since $\beta>\alpha_{n}$ implies $(z, \beta) \notin V(\{n\})$. Therefore, $z \in W(T \backslash\{n\})$, again contradicting $x \in C(U)$.

Next we show that $\left(x, \alpha_{n}\right)$ in $(N, V)$ cannot be blocked by $N$ either. Otherwise there exist $\beta>$ $\alpha_{n}, z \gg x$ such that $(z, \beta) \in V(N)$. It follows using comprehensiveness that $\left(z, \alpha_{n}\right) \in V(N)$, implying that $\left(z, \alpha_{n}\right) \in U(M)$, again contradicting $x \in C(U)$. Thus $\left(x, \alpha_{n}\right) \in C(V)$. 
Note that the construction used shows that all core elements of the reduced game can be extended to the original game.

Step II. If $S=\emptyset$ or $S=M$, then take any $x \in C(U)$ such that $x \in \partial U(T)$ for some $T \in$ $2^{M} \backslash\{\emptyset\}$. Note that such $x$ exists by applying the induction hypothesis. By Step I we have that $\left(x, \alpha_{n}\right) \in C(V)$. If $S=\emptyset$, then $\left(x, \alpha_{n}\right) \in \partial V(\{n\})$ by the definition of $\alpha_{n}$. If $S=M$, then $\left(x, \alpha_{n}\right) \in C(V)$ implies that $\left(x, \alpha_{n}\right) \in \partial V(N)=\partial V(M \cup\{n\})$.

If $S \neq \emptyset$ and $S \neq M$, then take any $x \in C(U)$ such that $x \in \partial U(S)$. Note that such $x$ exists by applying the induction hypothesis again. Since $S \neq \emptyset$ and $S \neq M$, we have that $U(S)=W(S) \cup P(S)$. So

$$
\begin{aligned}
x \in \partial(W(S) \cup P(S)) & =\operatorname{cl}(W(S) \cup P(S)) \cap \operatorname{cl}\left(\mathbb{R}^{M} \backslash(W(S) \cup P(S))\right) \\
& =(\operatorname{cl} W(S) \cup \operatorname{cl} P(S)) \cap \operatorname{cl}\left(\mathbb{R}^{M} \backslash(W(S) \cup P(S))\right) \\
& =(\partial W(S) \backslash \operatorname{int} P(S)) \cup(\partial P(S) \backslash \operatorname{int} W(S)),
\end{aligned}
$$

which implies that there are two (not exclusive) cases: $x \in \partial W(S) \backslash \operatorname{int} P(S)$ or $x \in \partial P(S) \backslash$ int $W(S)$.

Case 1: $x \in \partial W(S) \backslash$ int $P(S)$. Then, $x \in \partial W(S)$ implies $x \in \operatorname{cl} W(S) \cap \mathrm{cl}\left(\mathbb{R}^{M} \backslash W(S)\right)$. Since $x \in \operatorname{cl} W(S)$, there exists a sequence $\left(x^{k}\right)_{k \in \mathbb{N}}$ with $x^{k} \in W(S)$ for all $k \in \mathbb{N}$ and $\left(x^{k}\right)_{k \in \mathbb{N}} \rightarrow x$. Then, by the definition of $W(S)$, there exists a sequence $\left(\beta^{k}\right)_{k \in \mathbb{N}}$ with $\beta^{k}>\alpha_{n}$ and $\left(x^{k}, \beta^{k}\right) \in$ $V(S \cup\{n\})$ for all $k \in \mathbb{N}$. Due to comprehensiveness $\left(x^{k}, \alpha_{n}\right) \in V(S \cup\{n\})$ for all $k \in \mathbb{N}$ as well, and the sequence $\left(x^{k}, \alpha_{n}\right)_{k \in \mathbb{N}}$ converges to $\left(x, \alpha_{n}\right)$, implying that $\left(x, \alpha_{n}\right) \in \operatorname{cl} V(S \cup\{n\})$. Since $x \in \operatorname{cl}\left(\mathbb{R}^{M} \backslash W(S)\right)$ as well, there exists a sequence $\left(x^{k}\right)_{k \in \mathbb{N}}$ with $x^{k} \in \mathbb{R}^{M} \backslash W(S)$ for all $k \in \mathbb{N}$ and $\left(x^{k}\right)_{k \in \mathbb{N}} \rightarrow x$, that is for all $\beta>\alpha_{n}$ we have that $\left(x^{k}, \beta\right) \in \mathbb{R}^{N} \backslash V(S \cup\{n\})$ for all $k \in \mathbb{N}$. In particular, $\left(x^{k}, \alpha_{n}+1 /(k+1)\right) \in \mathbb{R}^{N} \backslash V(S \cup\{n\})$ for all $k \in \mathbb{N}$, and $\left(x^{k}, \alpha_{n}+1 /(k+1)\right)_{k \in \mathbb{N}} \rightarrow\left(x, \alpha_{n}\right)$, implying that $\left(x, \alpha_{n}\right) \in \operatorname{cl}\left(\mathbb{R}^{N} \backslash V(S \cup\{n\})\right)$. So $\left(x, \alpha_{n}\right) \in$ $\operatorname{cl} V(S \cup\{n\}) \cap \operatorname{cl}\left(\mathbb{R}^{N} \backslash V(S \cup\{n\})\right)$, thus $\left(x, \alpha_{n}\right) \in \partial V(S \cup\{n\})$.

Case 2: $x \in \partial P(S) \backslash$ int $W(S)$. By ordinal convexity of $(N, V)$ we have $V(S) \cap V(\{n\}) \subseteq$ $V(S \cup\{n\})$, which together with $x \in \partial P(S)$ implies that there exists a sequence $\left(x^{k}, \alpha_{n}^{k}\right)_{k \in \mathbb{N}}$ with $\left(x^{k}, \alpha_{n}^{k}\right) \in V(S \cup\{n\})$ for all $k \in \mathbb{N}$ and $\left(x^{k}, \alpha_{n}^{k}\right)_{k \in \mathbb{N}} \rightarrow\left(x, \alpha_{n}\right)$, so $\left(x, \alpha_{n}\right) \in \operatorname{cl} V(S \cup\{n\})$. Since $x \notin$ int $W(S)$, for all $y \gg x$ and for all $\beta>\alpha_{n}$ we have $(y, \beta) \notin V(S \cup\{n\})$. Thus there exists a sequence $\left(x^{k}, \alpha_{n}+1 /(k+1)\right)_{k \in \mathbb{N}} \rightarrow\left(x, \alpha_{n}\right)$ such that $\left(x^{k}, \alpha_{n}+1 /(k+1)\right) \in$ 
$\mathbb{R}^{N} \backslash V(S \cup\{n\})$, implying that $\left(x, \alpha_{n}\right) \in \operatorname{cl}\left(\mathbb{R}^{N} \backslash V(S \cup\{n\})\right)$. So $\left(x, \alpha_{n}\right) \in \operatorname{cl} V(S \cup\{n\}) \cap$ $\operatorname{cl}\left(\mathbb{R}^{N} \backslash V(S \cup\{n\})\right)$, thus $\left(x, \alpha_{n}\right) \in \partial V(S \cup\{n\})$.

Step III. In Step II we have established that for each $S \in 2^{M}$ there exists an $x$, such that $\left(x, \alpha_{n}\right) \in C(V)$ and $\left(x, \alpha_{n}\right) \in \partial V(S \cup\{n\})$. Instead of using player $n$, we could do the same construction with player $i$, implying that for each $S \in 2^{M}$ there exists an $x$, such that $\left(x, \alpha_{i}\right) \in C(V)$ and $\left(x, \alpha_{i}\right) \in \partial V(S \cup\{i\})$. To show that for each $T \in \mathcal{N}$ there exists a $y \in C(V)$ such that $y \in \partial V(T)$, choose $i$ to be any member of $T$ and let $S=T \backslash\{i\}$. Then, there exists an $x$, such that $\left(x, \alpha_{i}\right) \in C(V)$ and $\left(x, \alpha_{i}\right) \in \partial V(S \cup\{i\})$, so by setting $y=\left(x, \alpha_{i}\right)$ we have that $y \in C(V)$ and $y \in \partial V(T)$.

By Theorem 5.14, to verify whether the marginalistic interpretations of NTU convexity imply NTU exactness, it is enough to analyze marginal convexity.

Theorem 5.23. If an NTU game $(N, V)$ is marginal convex, then it is exact, $\Gamma_{\mathrm{mc}}^{\mathrm{NTU}} \subseteq \Gamma_{\mathrm{e}}^{\mathrm{NTU}}$.

Proof. Consider a marginal convex NTU game $(N, V)$, and a coalition $S \in \mathcal{N}$. For exactness we have to show that there is a core element on the boundary of $V(S)$. Let $\bar{\sigma}$ be a permutation such that $S \in\{\bar{\sigma}(1),\{\bar{\sigma}(1), \bar{\sigma}(2)\},\{\bar{\sigma}(1), \bar{\sigma}(2), \bar{\sigma}(3)\}, \ldots, N\}$. Since $(N, V)$ is marginal convex, we have that $M^{\bar{\sigma}}(V) \in C(V)$. By definition, $M^{\bar{\sigma}}(V)$ is on the boundary of $V(T)$ for all $T \in\{\bar{\sigma}(1),\{\bar{\sigma}(1), \bar{\sigma}(2)\},\{\bar{\sigma}(1), \bar{\sigma}(2), \bar{\sigma}(3)\}, \ldots, N\}$, thus it is a core element on the boundary of $V(S)$ as well.

Using Theorems 5.14 and 5.23 we have the following corollary.

Corollary 5.24. Each of coalition merge convexity, individual merge convexity and marginal convexity implies exactness in the NTU setting, $\Gamma_{\mathrm{cmc}}^{\mathrm{NTU}} \subseteq \Gamma_{\mathrm{imc}}^{\mathrm{NTU}} \subseteq \Gamma_{\mathrm{mc}}^{\mathrm{NTU}} \subseteq \Gamma_{\mathrm{e}}^{\mathrm{NTU}}$.

\subsection{Conclusion}

In this chapter we have generalized exactness to games with non-transferable utility to get the class of NTU exact games $\left(\Gamma_{\mathrm{e}}^{\mathrm{NTU}}\right)$. In an exact NTU game for each coalition there is a core allocation on the boundary of its payoff set, meaning that this coalition does not necessarily benefit from the gains of forming the grand coalition in an allocation which is robust against all 
coalitional deviations. We have noted that NTU exact games are a subset of totally $\Pi$-balanced NTU games $\left(\Gamma_{\mathrm{t}-\Pi-\mathrm{b}}^{\mathrm{NTU}}\right)$, having a non-empty core in each of their subgames.

We have shown that the classes of ordinally convex $\left(\Gamma_{\mathrm{oc}}^{\mathrm{NTU}}\right)$, coalition merge convex $\left(\Gamma_{\mathrm{cmc}}^{\mathrm{NTU}}\right)$, individual merge convex $\left(\Gamma_{\mathrm{imc}}^{\mathrm{NTU}}\right)$ and marginal convex $\left(\Gamma_{\mathrm{mc}}^{\mathrm{NTU}}\right)$ NTU games are a subset of NTU exact games.

Hendrickx, Borm, and Timmer (2002) show that the aforementioned five classes of NTU convex games do not coincide in general. The only general result (Theorem 5.14) is that coalition merge convexity implies individual merge convexity $\left(\Gamma_{\mathrm{cmc}}^{\mathrm{NTU}} \subseteq \Gamma_{\mathrm{imc}}^{\mathrm{NTU}}\right.$ ), and individual merge convexity implies marginal convexity $\left(\Gamma_{\mathrm{imc}}^{\mathrm{NTU}} \subseteq \Gamma_{\mathrm{mc}}^{\mathrm{NTU}}\right)$.

Theorem 5.6 claims that the class of convex TU games coincides with the class of totally exact TU games. In the NTU setting we do not have such a theorem. Let $\Gamma_{\text {te }}^{\mathrm{NTU}}$ denote the class of totally exact NTU games with $n$ players. Since an ordinally convex game is exact, and all subgames of an ordinally convex game are ordinally convex, we have that $\Gamma_{\mathrm{oc}}^{\mathrm{NTU}} \subseteq \Gamma_{\text {te }}^{\mathrm{NTU}}$. For marginal convex games a similar argument leads to $\Gamma_{\mathrm{mc}}^{\mathrm{NTU}} \subseteq \Gamma_{\mathrm{te}}^{\mathrm{NTU}}$.

However, using our results it is easy to provide counterexamples where NTU total exactness implies none of the NTU convexity notions. For instance, the NTU game in Example 5.15 is ordinally convex, and as we argued that game is totally NTU exact. But it is neither cardinal, nor marginal, nor individual merge, nor coalition merge convex. So neither cardinal, nor marginal, nor individual merge, nor coalition merge convexity is implied by total NTU exactness in general. Hendrickx, Borm, and Timmer (2000) provide an example (Example 4.6 there) for an NTU game which is marginal convex but not ordinally convex. That example can be used to show that total NTU exactness does not imply ordinal convexity either.

Analogously to TU games in Figure 3.1, we summarize the relationships between the various classes of NTU games in Figure 5.1. 


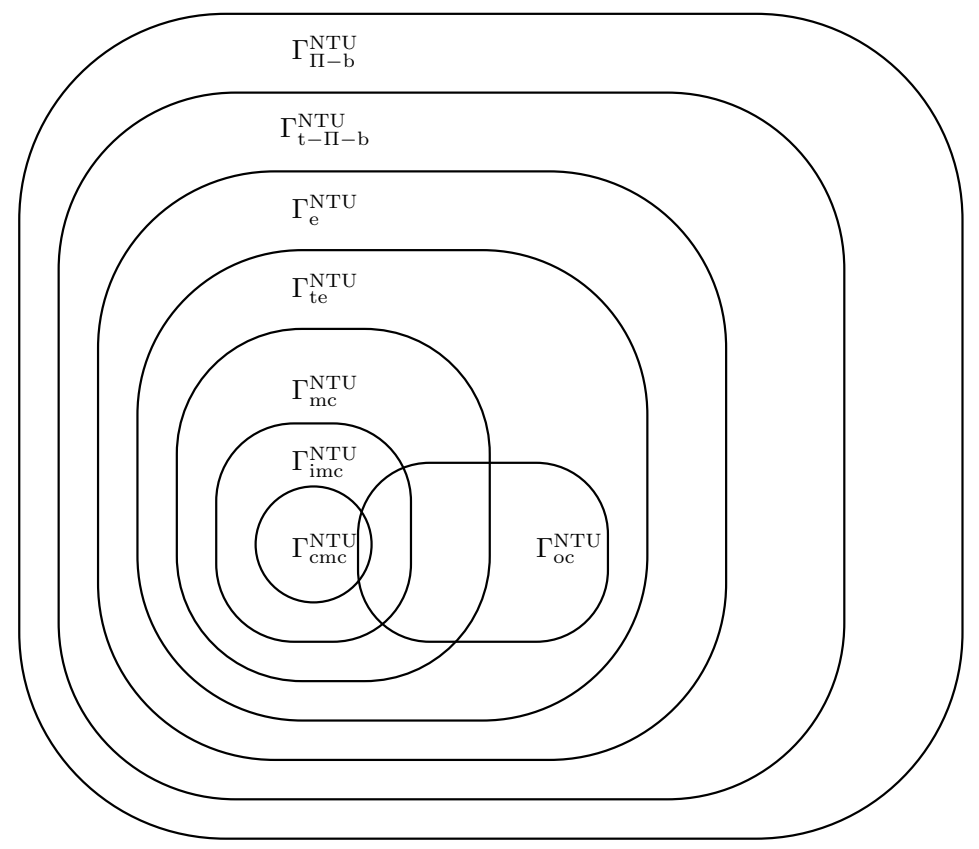

Figure 5.1: Subsets of П-balanced games. 


\section{Summary}

To have an adequate determination of capital requirements in the banking industry and a proper performance evaluation of portfolio managers it is crucial to measure and allocate risk in an appropriate way.

A measure of risk assigns a real number to the probability distribution of the future value of a portfolio. It can be interpreted as the minimal amount of cash the regulated agent has to add to his portfolio, and to invest in a zero coupon bond for its risk to be acceptable to the regulator. The literature knows of numerous possible ways to measure risk; lately interest shifted to coherent measures of risk (Artzner, Delbaen, Eber, and Heath, 1999) satisfying the following four axioms: monotonicity, subadditivity, positive homogeneity and translation invariance. Adding two more axioms, comonotonic additivity and law invariance, one obtains a subclass of coherent measures of risk, called spectral measures of risk.

Most importantly, subadditivity requires that the risk of an aggregate portfolio should not exceed the total risk of the individual portfolios: it captures the notion of diversification. The diversification benefits should be allocated somehow, preferably in a stable way, when no collection of individual portfolios would be better off if they separate from the others.

In this thesis we analyze the aforementioned axioms using tools from general equilibrium theory and study the allocation of risk by means of cooperative game theory.

Chapter 2 of the thesis elaborates on analyzing the axioms of coherent and spectral measures of risk using an exchange economy model. By doing so we contribute to the research agenda that connects finance to general equilibrium theory. The corresponding measure of risk, the so-called $G E$ measure of risk of a portfolio is the negative of its equilibrium market price in the exchange economy.

We prove that the GE measure of risk is a coherent measure of risk, thus coherent measures of risk are compatible with a natural general equilibrium approach to measure risk. However, using 
the insight of the Capital Asset Pricing Model (Sharpe, 1964; Lintner, 1965) that the risk of a portfolio does not only depend on the probability distribution of its payoff (law invariance), but also on how these payoffs are correlated to those of the market portfolio, we show that the GE measure of risk does not satisfy law invariance, and is therefore not a spectral measure of risk. We model the problem of risk allocation by cooperative game theory. A transferable utility (TU) game consists of a finite set of players and a value function specifying the maximum attainable utility (money) for all the coalitions of players. The core of a TU game consists of those efficient allocations which are robust against all coalitional deviations. One obtains a subgame by restricting the game to a subset of players. Games having a non-empty core in every subgame are called totally balanced.

In Chapter 3 we provide a set of linear programming problems to study a subclass of totally balanced games having a nice core structure, exact games (Schmeidler, 1972). A game is exact if for each coalition there is a core allocation such that the coalition only gets its stand-alone value. By the linear programming problems one can easily check whether a game is exact or not. Using the dual of the linear programming problems we develop two new characterizations of exact games, complementing earlier characterizations by Schmeidler (1972) and Azrieli and Lehrer (2005). The characterizations can be used to verify exactness of a game, we apply them in Chapter 4.

In Chapter 4 we come back to the question of the distribution of the risk diversification benefits. Risk allocation games (Denault, 2001) are transferable utility cooperative games defined to this purpose. A risk allocation game arises from a risk environment specifying a number of portfolios and a coherent measure of risk determining the risk of each portfolio. Coalitions of agents can combine their portfolios and thereby create diversification gains.

We prove that the class of risk allocation games coincides with the class of totally balanced games. This result ensures that a regulator can always allocate risk in a stable way. No matter how the risk environment changes, there is always an allocation of risk that no coalition of portfolios can object to.

To get exact games, we need an extra condition, since exact games are a subclass of totally balanced games. Usually the risk of the aggregate portfolio is low compared to the risk involved in the individual portfolios. As an extreme case, no aggregate uncertainty refers to the case when the value of the aggregate portfolio is constant over all states of nature. We prove that the class 
of risk allocation games with no aggregate uncertainty coincides with the class of exact games. To show that all risk allocation games with no aggregate uncertainty are exact, we use one of the characterizations of exact games developed in Chapter 3.

Thus, in the case of no aggregate uncertainty, for each coalition of portfolios, this coalition does not necessarily benefit from the diversification opportunities offered by the aggregate portfolio. As a consequence, the regulator has a high level of discretion in allocating the risk to the individual portfolios in this case.

Convex games with transferable utility introduced by Shapley (1971) provide a further refinement of exact games, since convex games are a subset of exact games. If the number of players (individual portfolios) below four, we show that one obtains the class of convex games in risk allocation games with no aggregate uncertainty.

The assumption of transferable utility can be relaxed to obtain the class of games with nontransferable utility (NTU games, for short). In a TU game it is assumed that utilities can be transferred. Such an assumption is justified if there is a commodity which has the same marginal utility for everyone and the utility functions are linear and separable in it. In general, this is not the case and one would like to study the more general class of NTU games. An NTU game specifies the set of attainable utility levels for the members of each coalition.

The notion of convexity can be generalized to NTU games in at least five ways. Vilkov (1977) and Sharkey (1981) have extended convexity to NTU games to define ordinal and cardinal convexity, respectively. Hendrickx, Borm, and Timmer (2002) analyze coalition merge convexity, individual merge convexity, and marginal convexity in an NTU setting.

The aforementioned five classes of NTU convex games do not coincide in general. The only general result is that coalition merge convexity implies individual merge convexity, and individual merge convexity implies marginal convexity. It is natural to seek a result that is analogous to convex TU games being exact. In Chapter 5 we generalize exactness to the NTU setting. In an exact NTU game for each coalition there is a core element on the boundary of its payoff set, meaning that this coalition does not necessarily benefit from the gains of forming the grand coalition in an allocation which is robust against all coalitional deviations. We show that each of ordinal, coalition merge, individual merge and marginal convexity can be unified under NTU exactness, which gives a sensible, common property of the core for all those games. 


\section{Bibliography}

Acerbi, C., Spectral measures of risk: A coherent representation of subjective risk aversion. Journal of Banking and Finance, 26, (2002), pp. 1505-1518.

Acerbi, C. (2004). Coherent representations of subjective risk aversion. In: G. Szegö (Editor), Risk Measures for the 21 st Century, pp. 149-207. Wiley, New York.

Acerbi, C. and D. Tasche, On the coherence of expected shortfall. Journal of Banking and Finance, 26, (2002), pp. 1487-1504.

Artzner, P. F., F. Delbaen, J.-M. Eber, and D. Heath, Coherent measures of risk. Mathematical Finance, 9, (1999), pp. 203-228.

Aumann, R. and M. Maschler, Game theoretic analysis of a bankruptcy problem from the Talmud. Journal of Economic Theory, 36, (1985), pp. 195-213.

Azrieli, Y. and E. Lehrer, Concavification and convex games. Working Paper. School of Mathematical Sciences, Tel Aviv University.

Biswas, A. K., T. Parthasarathy, J. A. M. Potters, and M. Voorneveld, Large cores and exactness. Games and Economic Behavior, 28, (1999), pp. 1-12.

Bondareva, O. N., Some applications of linear programming methods to the theory of cooperative games (in Russian). Problemy Kybernetiki, 10, (1963), pp. 119-139.

Calleja, P., P. Borm, and R. Hendrickx, Multi-issue allocation situations. European Journal of Operational Research, 164, (2005), pp. 730-747.

Csóka, P., P. J. J. Herings, and L. Á. Kóczy, Balancedness conditions for exact games. RM07/040, (2007a), pp. 1-13. METEOR Research Memorandum. 
Csóka, P., P. J. J. Herings, and L. Á. Kóczy, Coherent measures of risk from a general equilibrium perspective. Journal of Banking and Finance, 31, no. 8, (2007b), pp. 2517-2534. doi:10.1016/j.jbank.n.2006.10.026.

Csóka, P., P. J. J. Herings, and L. Á. Kóczy, Stable allocations of risk. RM07/041, (2007c), pp. 1-19. METEOR Research Memorandum.

Curiel, I., G. Pederzoli, and S. Tijs, Sequencing games. European Journal of Operational Research, 40, (1989), pp. 344-351.

Demange, G., Nonmanipulable cores. Econometrica, 55, no. 5, (1987), pp. 1057-1074.

Denault, M., Coherent allocation of risk capital. Journal of Risk, 4, no. 1, (2001), pp. 1-34.

Derks, J. and H. Reijnierse, On the core of a collection of coalitions. International Journal of Game Theory, 27, (1998), pp. 451-459.

Dubey, P. and L. S. Shapley, Totally balanced games arising from controlled programming problems. Mathematical Programming, 29, (1984), pp. 245-267.

Duffie, D. (2001). Dynamic Asset Pricing Theory, Third Edition. Princeton University Press, Princeton.

Embrechts, P., A. McNeil, and D. Straumann (2002). Correlation and dependence in risk management: Properties and pitfalls. In: M. A. H. Dempster (Editor), Risk Management: Value at Risk and Beyond, pp. 176-223. Cambridge University Press, Cambridge.

Föllmer, H. and H. Schied (2002). Stochastic Finance: An Introduction in Discrete Time. Walter de Gruyter, Berlin.

Geanakoplos, J. and M. Shubik, The capital asset pricing model as a general equilibirum with incomplete markets. The Geneva Papers on Risk and Insurance, 15, (1990), pp. 55-72.

Granot, D., M. Maschler, G. Owen, and W. Zhu, The kernel/nucleolus of a standard tree game. International Journal of Game Theory, 25, (1996), pp. 219-244.

Greenberg, J., Cores of convex games without side payments. Mathematics of Operations Research, 10, no. 3, (1985), pp. 523-525. 
Hendrickx, R., P. Borm, and J. Timmer, On convexity for NTU-games. Discussion Paper 108, Tilburg University, Center for Economic Research.

Hendrickx, R., P. Borm, and J. Timmer, A note on NTU convexity. International Journal of Game Theory, 31, (2002), pp. 29-37.

Ibragimov, R., Portfolio diversification and value at risk under thick-tailedness. Harvard Institute of Economic Research Discussion Paper 2086.

Ichiishi, T., Super-modularity: Applications to convex games and the greedy algorithm for LP. Journal of Economic Theory, 25, (1981), pp. 283-286.

Jaschke, S. and U. Küchler, Coherent risk measures and good-deal bounds. Finance and Stochastics, 5, (2001), pp. 181-200.

Kalai, E. and E. Zemel, Generalized network problems yielding totally balanced games. Operations Research, 30, (1982a), pp. 998-1008.

Kalai, E. and E. Zemel, Totally balanced games and games of flow. Mathematics of Operations Research, 7, (1982b), pp. 476-478.

Legut, J., On totally balanced games arising from cooperation in fair division. Games and Economic Behavior, 2, (1990), pp. 47-60.

Leroy, S. F. and J. Werner (2001). Principles of Financial Economics. Cambridge University Press, Cambridge.

Lintner, J., The valuation of risky assets and the selection of risky investments in stock portfolios and capital budgets. Review of Economics and Statistics, 47, (1965), pp. 13-37.

Littlechild, S. and G. Owen, A simple expression for the Shapley value in a special case. Management Science, 20, (1973), pp. 370-372.

Magill, M. J. M. and M. Quinzii (1996). Theory of Incomplete Markets. MIT Press, Cambridge, Massachusetts.

Marshall, A. W. and I. Olkin (1979). Inequalities: Theory of Majorization and Its Applications. Academic Press, New York. 
Mas-Colell, A., M. D. Whinston, and J. R. Green (1995). Microeconomic Theory. Oxford University Press, Oxford.

Masuzawa, T., Punishment strategies make the $\alpha$-coalitional game ordinally convex and balanced. International Journal of Game Theory, 32, (2003), pp. 479-483.

Nelsen, R. B. (1999). An Introduction to Copulas, volume 139 of Lecture Notes in Statistics. Springer-Verlag, New York.

Owen, G., On the core of linear production games. Mathematical Programming, 9, (1975), pp. $358-370$.

Peleg, B. (1984). Game Theoretic Analysis of Voting in Comittees. Cambridge University Press.

Peleg, B., A proof that the core of an ordinal convex game is a von Neumann-Morgenstern solution. Mathematical Social Sciences, 11, (1986), pp. 83-87.

Predtetchinski, A. and P. J. J. Herings, A necessary and sufficient condition for the non-emptiness of the core of a non-transferable utility game. Journal of Economic Theory, 116, (2004), pp. 84-92.

Schmeidler, D., Cores of exact games. Journal of Mathematical Analysis and Applications, 40, (1972), pp. 214-225.

Shapley, L. S., On balanced sets and cores. Naval Research Logistics Quarterly, 14, (1967), pp. 453-460.

Shapley, L. S., Cores of convex games. International Journal of Game Theory, 1, (1971), pp. $11-26$.

Shapley, L. S. and M. Shubik, On market games. Journal of Economic Theory, 1, (1969), pp. $9-25$.

Sharkey, W., Convex games without side payments. International Journal of Game Theory, 10, (1981), pp. 101-106.

Sharpe, W. F., Capital asset prices: A theory of market equilibrium under conditions of risk. Journal of Finance, 19, (1964), pp. 425-442. 
Tasche, D., Expected shortfall and beyond. Journal of Banking and Finance, 26, (2002), pp. $1519-1533$.

Tijs, S., T. Parthasarathy, J. Potters, and V. R. Prassad, Permutation games: Another class of totally balanced games. OR Spektrum, 6, (1984), pp. 119-123.

Vilkov, V., Convex games without side payments (in Russian). Vestnik Leningradskiva Universitata, 7, (1977), pp. 21-24.

von Neumann, J. and O. Morgenstern (1944). Theory of Games and Economic Behavior. Princeton University Press. 


\section{Index}

П-balancedness, 69

Acerbi, Carlo, 2, 8, 11, 13, 14, 48, 49

Allocation, 33, 49

coalitionally rational, 33, 49, 67

efficient, 33, 49, 67

individually rational, 33, 49, 67, 70

weakly Pareto efficient, 70

Artzner, Philippe, 1, 8, 10, 46, 48, 65

Aumann, Robert J., 6, 65

Azrieli, Yaron, 4, 40

Balancedness, 4, 50

Biswas, Amit K., 32, 34, 62, 68

Bondareva, Olga Nikolajevna, 4, 31, 33, 50

Borm, Peter, 6, 47, 66, 70-73, 78

Calleja, Pedro, 47

Coalition, 33, 66

Competitive equilibrium, 3,19

Contingent contract, 18

Core, 4, 33, 49, 67

of an NTU game, 69

Core requirement, 56

Curiel, Imma, 6, 65

Delbaen, Freddy, 1, 8, 10, 46, 48, 65

Demange, Gabrielle, 6, 66
Denault, Michel, 5, 46, 51, 64

Derks, Jean, 39, 50

Dubey, Pradeep, 32, 46

Duffie, Darrell, 8

Eber, Jean-Marc, 1, 8, 10, 46, 48, 65

Economy, 18

exchange, 2

Embrechts, Paul, 11

Föllmer, Hans, 2, 8

Game

П-balanced, 69

additive, 56

balanced, 33, 50

cardinally convex, 6, 66, 70

coalition merge convex, $6,66,71$

convex, 5, 34, 51, 65, 67

convex for the grand coalition, 34

exact, 4, 34, 50, 65, 67

exactly balanced, 4,36

individual merge convex, 6, 66, 71

individually superadditive, 70

marginal convex, 6, 66, 71

monotonic, 70

NTU exact, 6, 66, 73 
ordinally convex, $6,66,70$

overbalanced, 5, 37

reduced, 74,76

risk allocation, 5, 52

superadditive, 70

totally balanced, 31, 34, 46, 50

totally exact, 34

with non-transferable utility, 6, 69

with transferable utility, 4, 33, 49, 67

zero normalized, 70

Game theory

cooperative, 3

noncooperative, 3

Geanakoplos, John, 3, 9, 20

General equilibrium theory, 2

Granot, Daniel, 6, 65

Green, Jerry R., 19

Greenberg, Joseph, 74

Heath, David, 1, 8, 10, 46, 48, 65

Hendrickx, Ruud, 6, 47, 66, 70-73, 78

Herings, Jean-Jacques, 50, 69

Ibragimov, Rustam, 11

Ichiishi, Tatsuro, 68

Jaschke, Stefan, 3, 9, 21

Küchler, Uwe, 3, 9, 21

Kalai, Ehud, 4, 32, 46, 56

Law invariance, 2, 14

Legut, Jerzy, 31, 46

Lehrer, Ehud, 4, 40
LeRoy, Stephen, 3, 9

Lintner, John, 3, 9

Littlechild, S. C., 6, 65

Magill, Michael, 3, 9

Majorization, 13

Marginal contribution, 68

Market portfolio, 20

Marshall, Albert W., 13

Mas-Colell, Andreu, 19

Maschler, Michael, 6, 65

Masuzawa, Takuya, 6, 66

McNeil, Alexander, 11

Measure of risk, 10, 48

coherent, 5, 10, 48

comonotonic additive, 14

expected shortfall, 12, 49

general equilibrium (GE), 3, 20

generalized law invariant, 22

law invariant, 2, 14

monotone, 10, 48

positive homogeneous, 10,48

spectral, 12, 49

subadditive, 2, 10, 48

translation invariant, 10, 48

with the pricing kernel property, 27

Monotonicity, 10, 48

Nelsen, Roger, 15

No aggregate uncertainty, 5, 52, 60

Olkin, Ingram, 13

Overbalancedness, 50 
Owen, Guillermo, 4, 6, 32, 46, 65

Parthasarathy, T., 4, 32, 34, 46, 62, 68

Pederzoli, Giorgio, 6, 65

Peleg, Bezalel, 6, 66, 74

Permutation, 68

Players, 33, 49, 66

Positive homogeneity, 10, 48

Potters, Curiel, J., 4, 32, 34, 46, 62, 68

Prassad, V. Rajendra, 4, 32, 46

Predtetchinski, Arkadi, 50, 69

Pricing kernel property, 27

Quinzii, Martine, 3, 9

Reduced game, 74, 76

Reijnierse, Hans, 39, 50

Risk, 45

allocation game, 5, 52

capital allocation problem, 51

environment, 52

management, 7

spectrum, 12, 49

Ross, Stephen, 3, 9
Shubik, Martin, 3, 4, 9, 20, 31, 46

Strauman, Daniel, 11

Subadditivity, 2, 10, 48

Subgame, 4, 33, 50, 68, 69

Tasche, Dirk, 8, 15, 49

Tijs, Stef, 4, 6, 32, 46, 65

Timmer, Judith, 6, 66, 70-73, 78

Total balancedness, 4, 34, 50

Total exactness, 68

Translation invariance, 10, 48

Value function, 33, 49, 67

Vector of marginal contributions, 68, 70

Vector of weights

balanced, 33, 50

exactly balanced, 36

overbalanced, 37,50

totally balanced, 34,50

Vilkov, V., 6, 66, 70

Von Neumann, John, 4

Voorneveld, Mark, 32, 34, 62, 68

Werner, Jan, 3, 9

Whinston, Michael D., 19

Zemel, Eitan, 4, 32, 46, 56

Schied, Alexander, 2, 8

Schmeidler, David, 4, 32, 34, 38, 41, 47, 50, 65,67

Schur-convex, 13

Shapley, Lloyd S., 4, 5, 31, 33, 46, 50, 51, 65, 67,68

Sharkey, W. W., 6, 66, 70, 72

Sharpe, William, 3, 9 


\section{Short Curriculum Vitae}

Péter Csóka was born on June 10, 1980 in Mátészalka, Hungary. He graduated from Sándor Petőfi Secondary School of Economics in 1998. Between 1998 and 2003, he studied Mathematical Economics and Finance, and majored as Investment Analyst at Budapest University of Economic Sciences and Public Administration (BUESPA, now Corvinus University). The academic year 2003/2004, Péter Csóka was a doctoral student at BUESPA. Between 2004 and 2008, he continued his doctoral studies at the Department of Economics of Maastricht University. His research was supervised by Prof. Dr. P. Jean-Jacques Herings and Dr. László Á. Kóczy.

Péter Csóka's research and teaching interests include game theory, risk management, general equilibrium theory, industrial organizations. 